Aus der Klinik für Unfallchirurgie und Orthopädie

- Abteilung Unfallchirurgie, Plastische- und Wiederherstellungschirurgie -

(Prof. Dr. med K. M. Stürmer)

im Zentrum Chirurgie

der Medizinischen Fakultät der Universität Göttingen

\title{
Einfluss der vertikalen Ganzkörpervibration verschiedener Frequenzen auf die Frakturheilung der osteoporotischen Ratte
}

\author{
INAUGURAL-DISSERTATION \\ zur Erlangung des Doktorgrades \\ der Medizinischen Fakultät der \\ Georg-August-Universität zu Göttingen
}

vorgelegt von

Lukas Michael Trautmann

aus

Göttingen

Göttingen 2014 
Dekan:

1. Berichterstatterin:

2. Berichterstatter:
Prof. Dr. rer. nat. H. K. Kroemer

Prof. Dr. med. E. K. Stürmer

Prof. Dr. med. Dr. med. dent. F.-J. Kramer

Tag der mündlichen Prüfung: 18. November 2014 


\section{Inhaltsverzeichnis}

\section{Abkürzungsverzeichnis}

1 Einleitung........................................................................................................................ 1

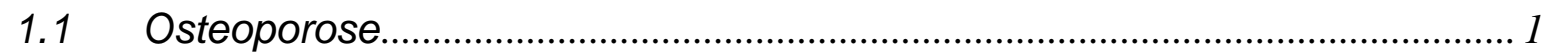

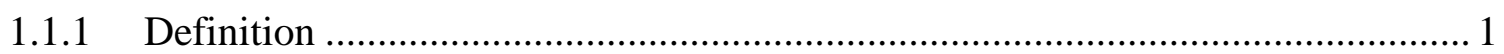

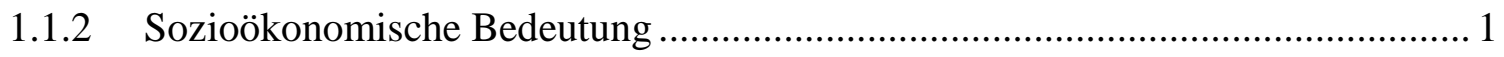

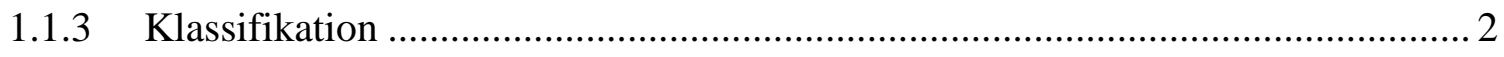

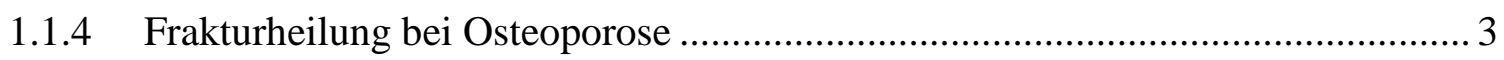

1.2 Allgemeine Standards der Osteoporosetherapie .......................................... 4

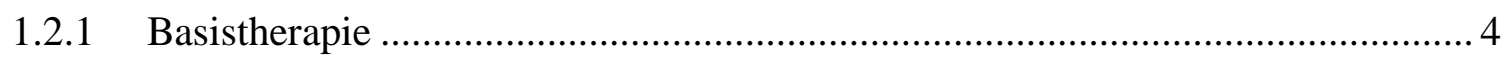

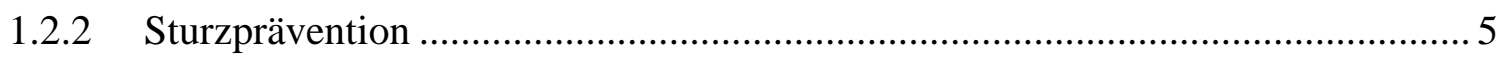

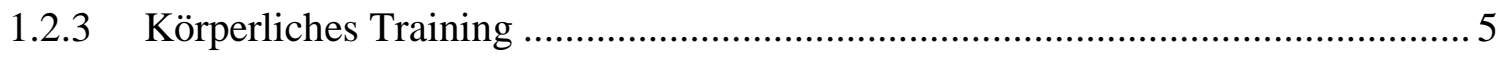

1.3 Medikamentöse Osteoporosetherapie ...................................................... 6

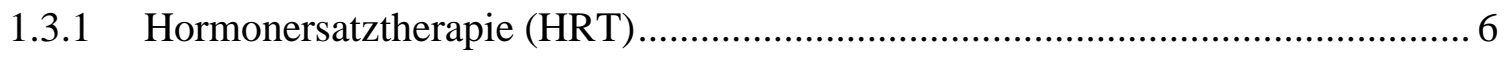

1.3.2 Selektive Östrogen-Rezeptor-Modulatoren (SERMs) ........................................ 7

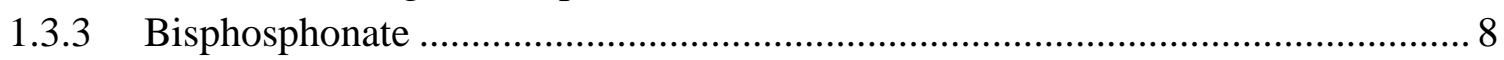

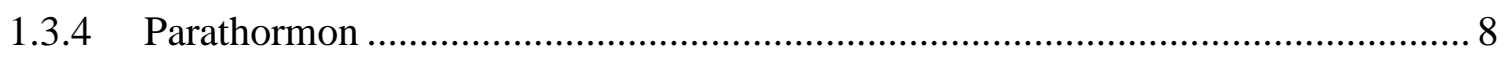

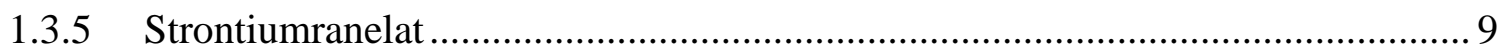

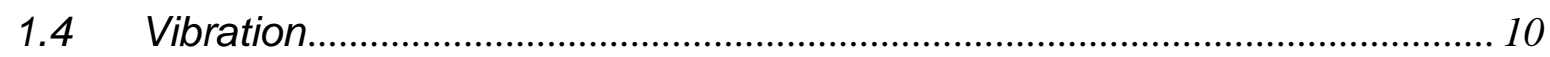

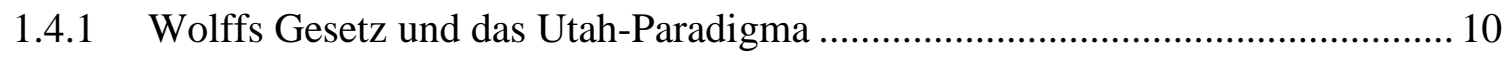

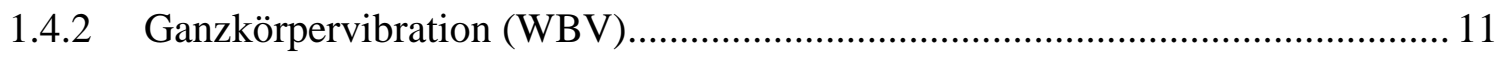

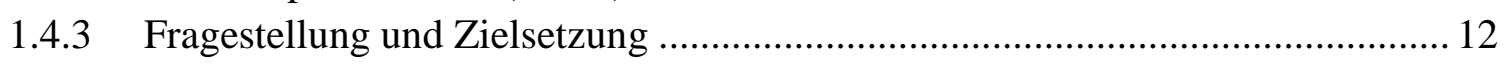

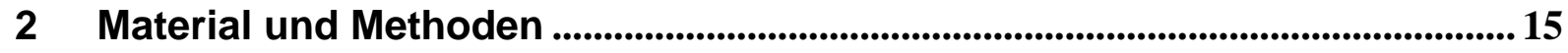

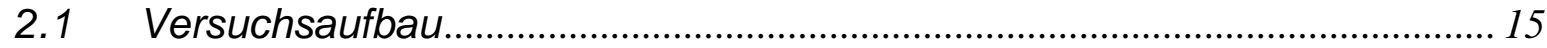

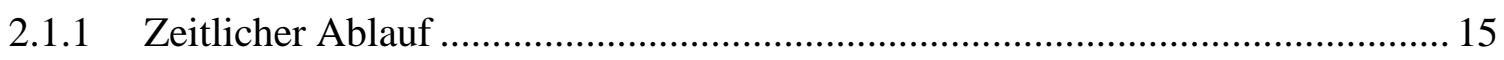

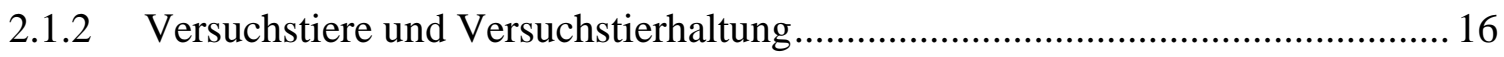

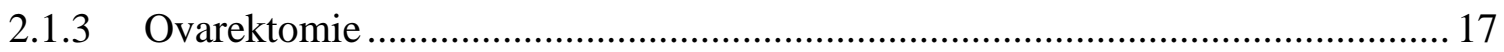

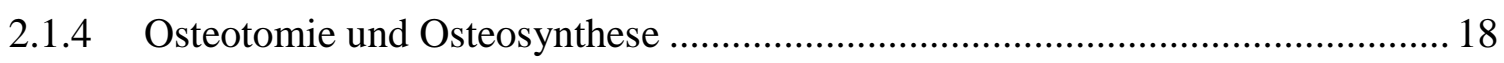

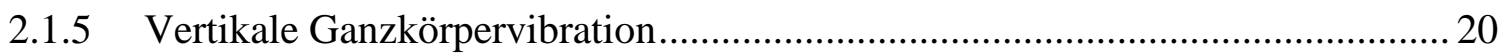

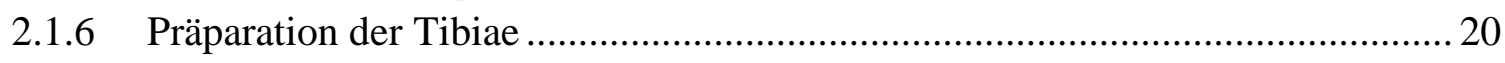

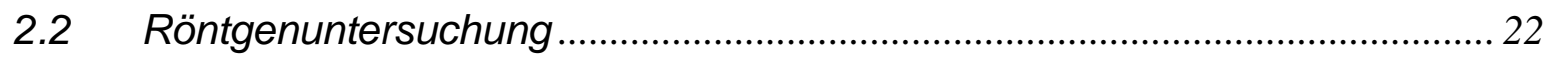

2.2.1 Technische Durchführung der Röntgenuntersuchung......................................... 22

2.2.2 Kriterien der röntgenologischen Befundung ................................................... 22

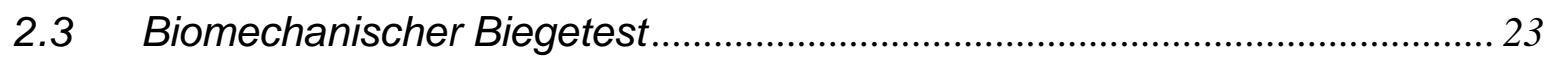

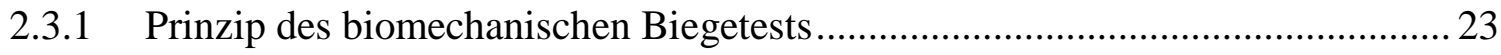

2.3.2 Versuchsaufbau des biomechanischen Biegetests........................................... 24

2.3.3 Messparameter des biomechanischen Biegetests ............................................ 26

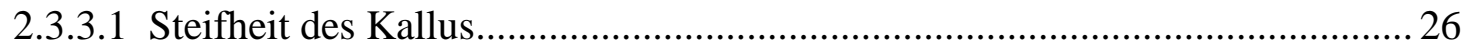

2.3.3.2 Maximale Belastbarkeit des Kallus ............................................................... 27

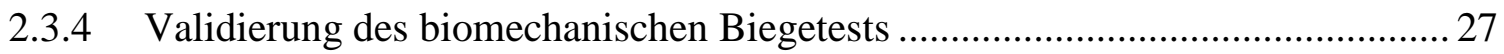




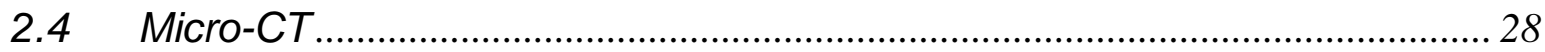

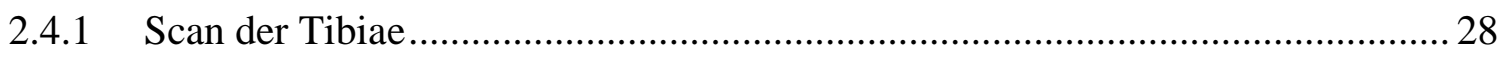

2.4.2 Morphometrische Auswertung der Micro-CT-Untersuchung............................. 29

2.4.3 Messparameter der Micro-CT-Untersuchung .................................................... 33

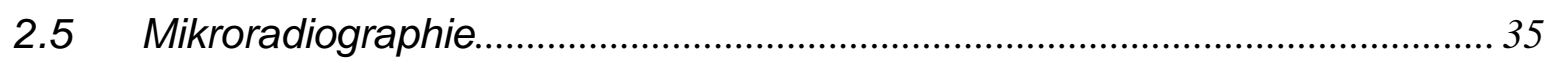

2.5.1 Histologische Aufarbeitung und Anfertigung der Mikroradiographien............... 35

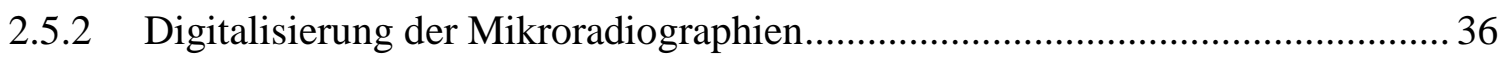

2.5.3 Morphometrische Auswertung der Mikroradiographien................................... 37

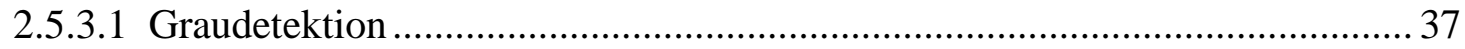

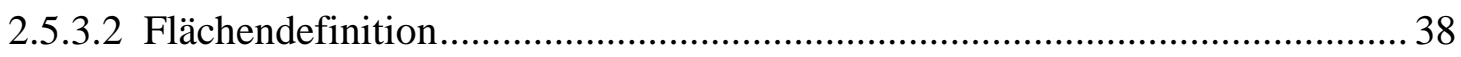

2.5.3.3 Vektorgestützte Knochendickemessung ......................................................... 40

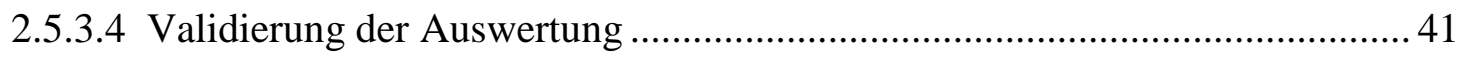

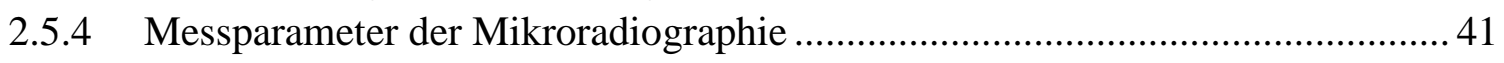

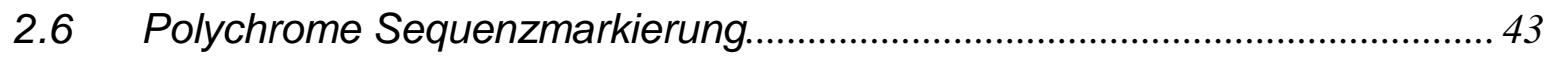

2.6.1 Prinzip der polychromen Sequenzmarkierung ............................................... 43

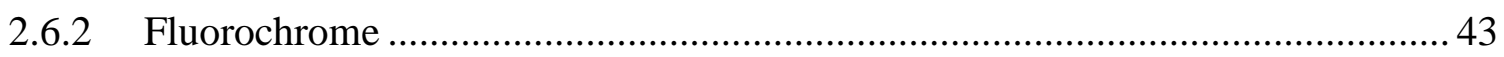

2.6.3 Digitalisierung der polychromen Sequenzmarkierung.................................... 44

2.6.4 Morphometrische Auswertung der polychromen Sequenzmarkierung............... 45

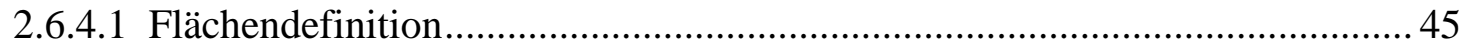

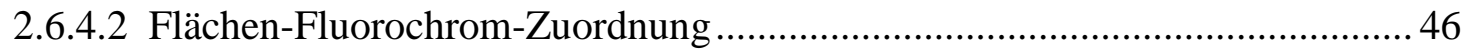

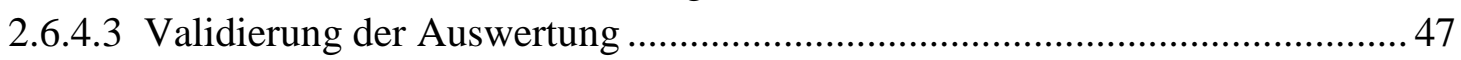

2.6.5 Messparameter der polychromen Sequenzmarkierung ..................................... 48

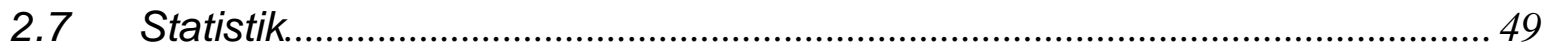

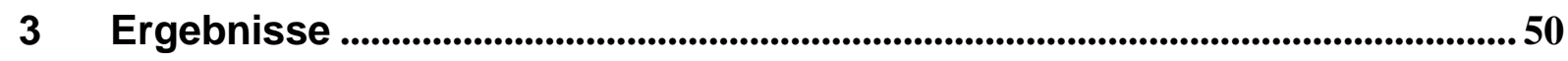

3.1 Futteraufnahme, Körpergewicht und Uterusgewicht der Tiere ...................... 50

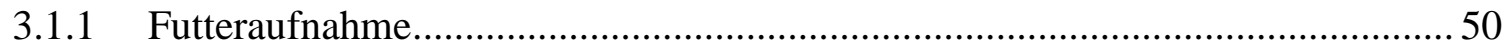

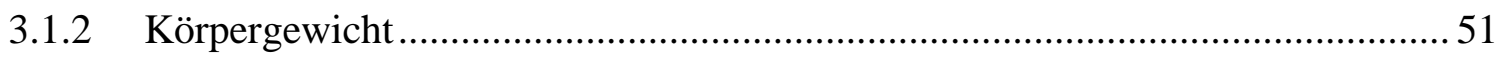

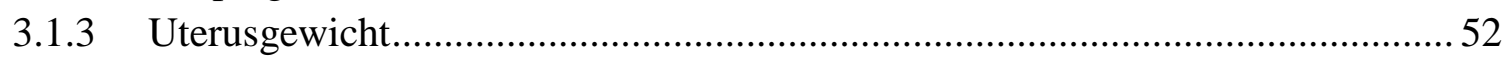

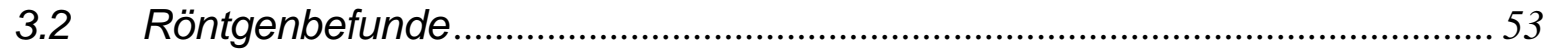

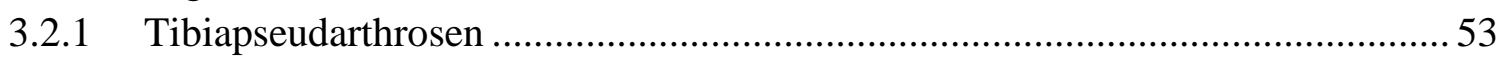

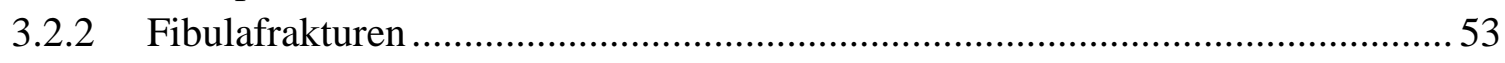

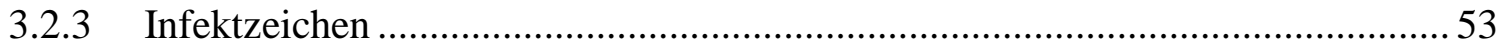

3.3 Ergebnisse des biomechanischen Biegetests ............................................ 54

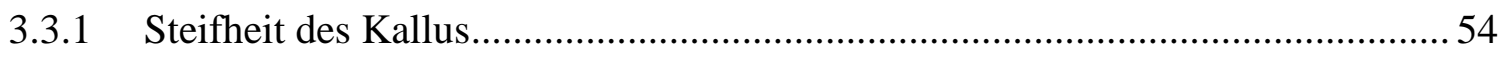

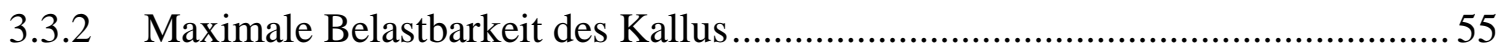

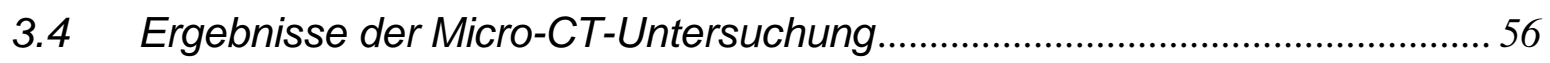

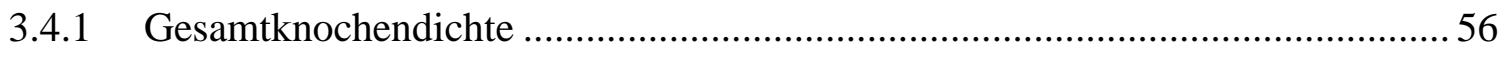

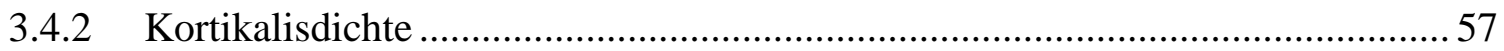

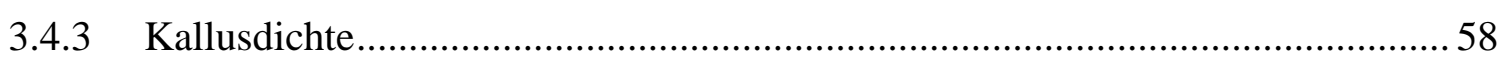

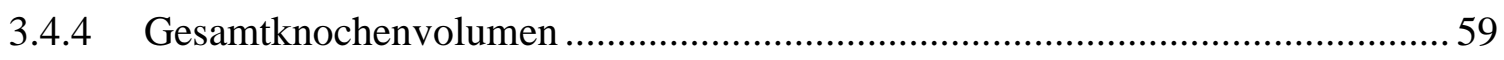

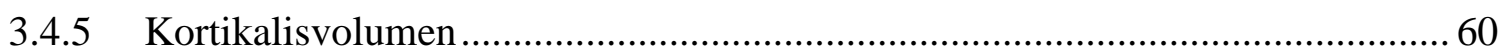

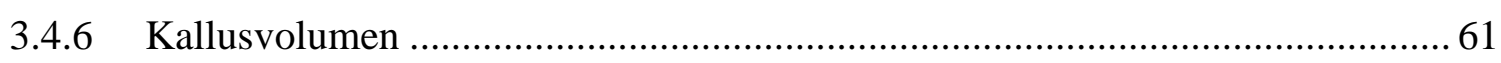




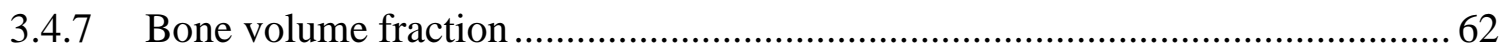

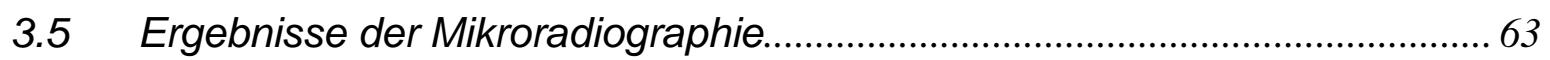

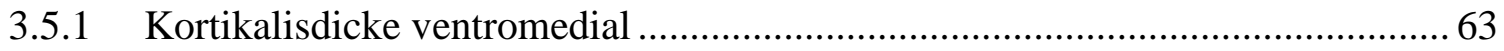

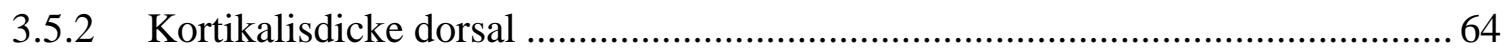

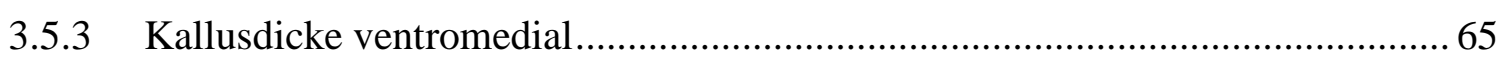

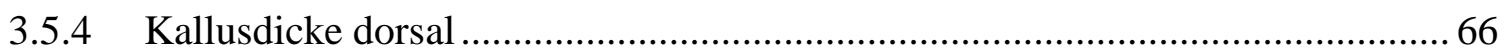

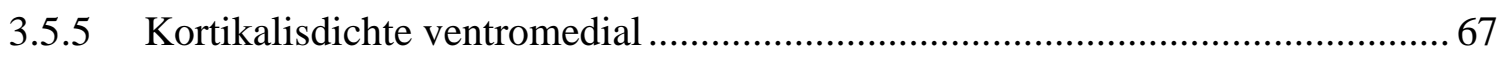

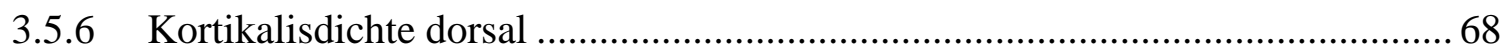

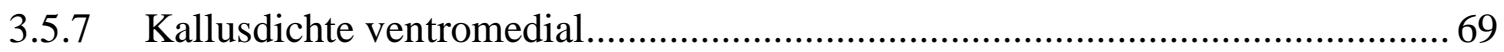

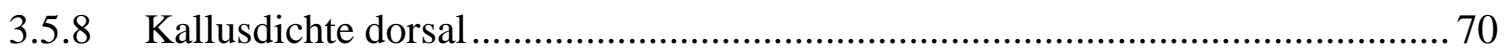

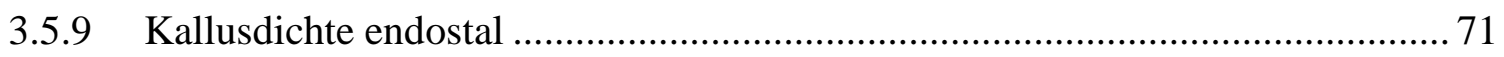

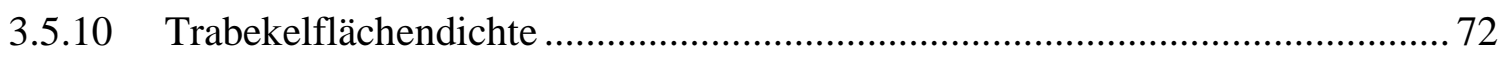

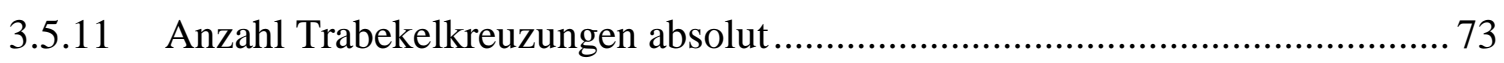

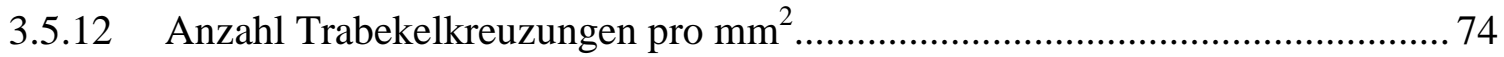

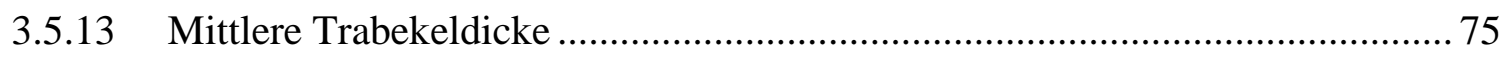

3.6 Ergebnisse der polychromen Sequenzmarkierung ........................................ 76

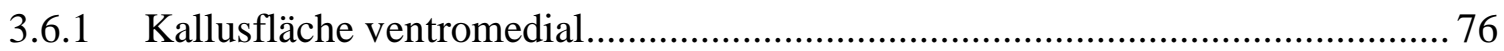

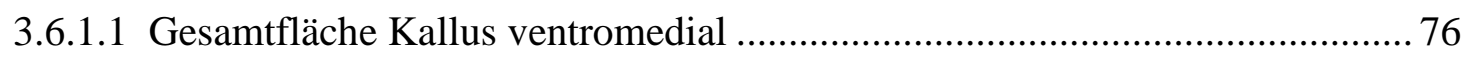

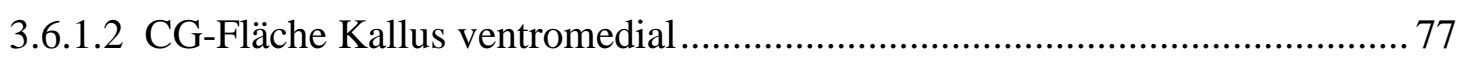

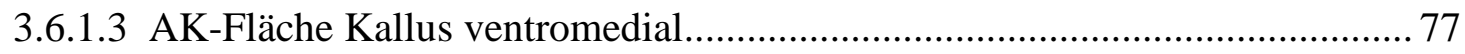

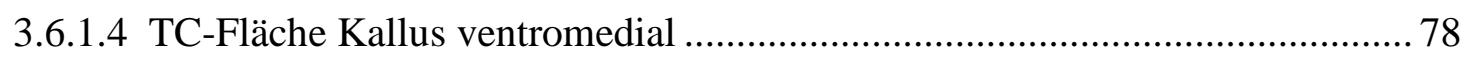

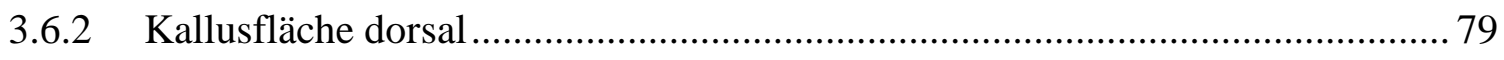

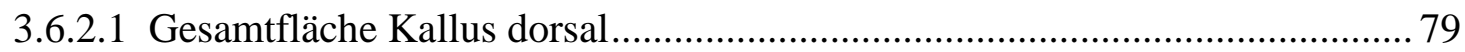

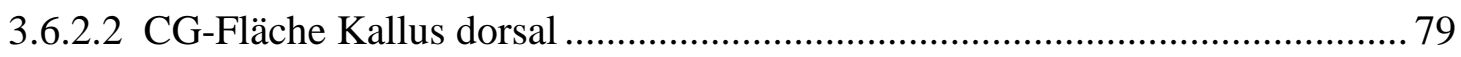

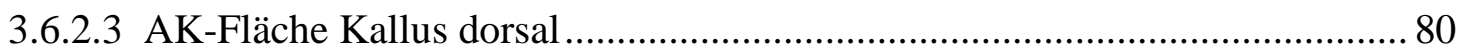

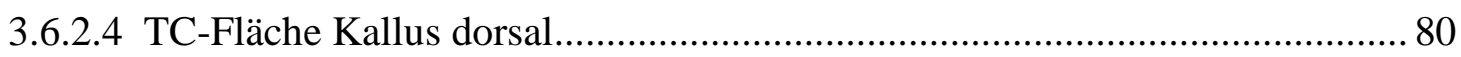

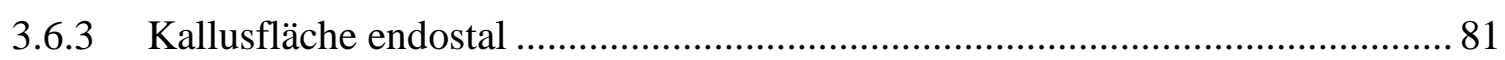

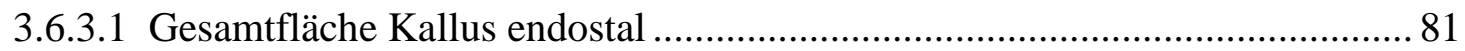

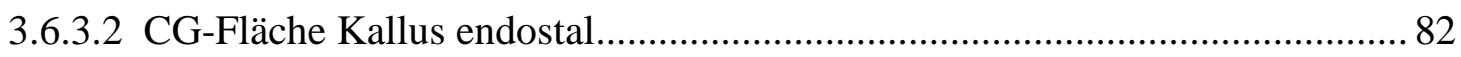

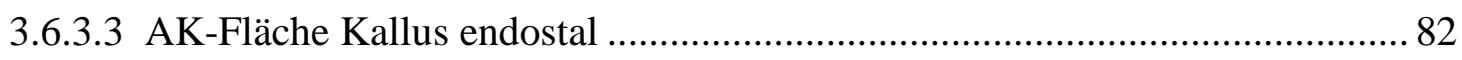

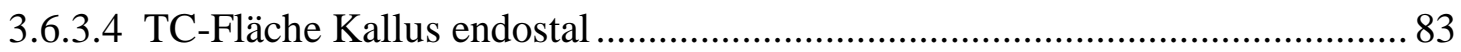

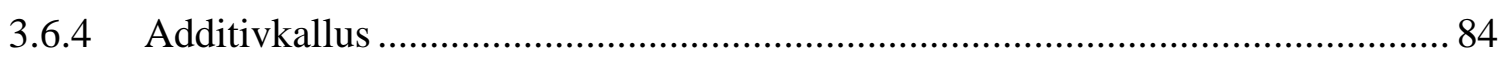

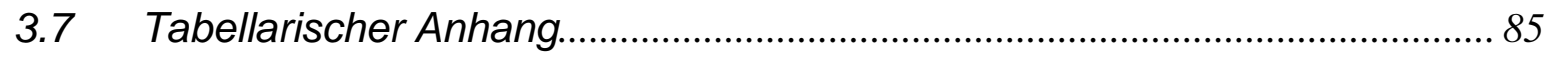

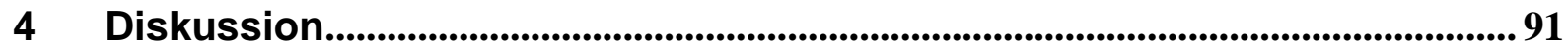

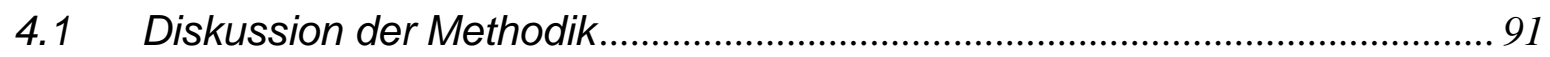

4.1.1 Die ovarektomierte Ratte als Osteoporosemodell ........................................... 91

4.1.2 Das metaphysäre Frakturmodell der ovarektomierten Ratte...............................91

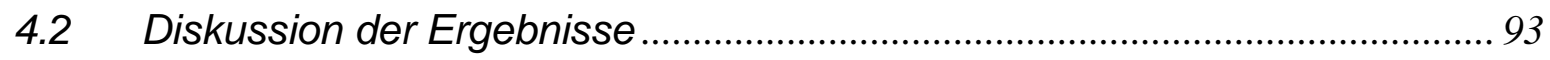

4.2.1 Qualitative Analyse der Frakturheilung unter vertikaler Ganzkörper- ............... 93

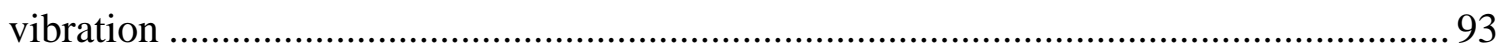

4.2.2 Quantitative Analyse der Frakturheilung unter vertikaler Ganzkörper- .............. 94

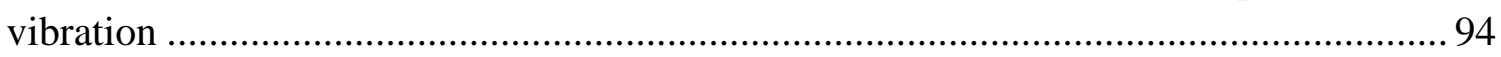

4.2.3 Chronologische Analyse der Frakturheilung unter vertikaler ............................. 99 


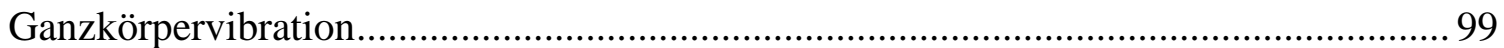

4.2.4 Vergleichende Analyse der Ergebnisse ....................................................... 100

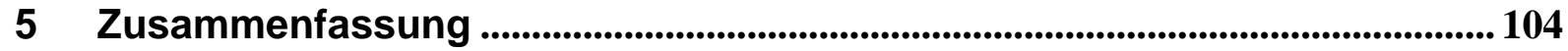

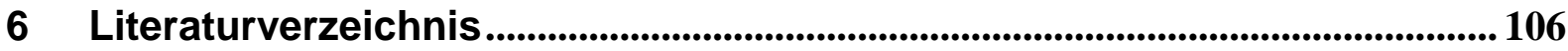

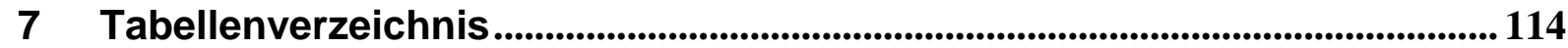

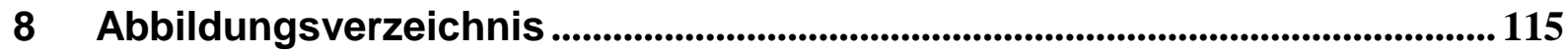




\section{Abkürzungsverzeichnis}

Abb.

AK

ap

$\mathrm{AZ}$

BMD

$\mathrm{BV} / \mathrm{TV}$

bzw.

${ }^{\circ} \mathrm{C}$

$\mathrm{ccm}$

CG

$\mathrm{cm}$

$\mathrm{cm}^{3}$

$\mathrm{CO}_{2}$

Co.KG

3-D

DFG

DXA

ER

et al.

e.V.

g

$\mathrm{GHz}$

$\mathrm{GmbH}$

HRT

$\mathrm{Hz}$

IE

Inc.

ISO

$\mathrm{K}$

KG
Abbildung

Alizarin-Komplexon

anterior-posterior (Strahlengang)

Aktenzeichen

Bone Mineral Density

Bone Volume/Total Volume = "bone volume fraction"

beziehungsweise

Grad Celsius

siehe $\mathrm{cm}^{3}$

Calcein-Grün

Zentimeter

Kubikzentimeter

Kohlendioxid

Compagnie Kommanditgesellschaft

dreidimensional

Deutsche Forschungsgemeinschaft

Dual Energy X-ray Absorptiometry

Estrogen Receptor

et alii = und andere (Autoren)

eingetragener Verein

Gramm

Gigahertz

Gesellschaft mit beschränkter Haftung

Hormone Replacement Therapy

Hertz

Internationale Einheit

Incorporated

International Organization for Standardization

Kelvin

Körpergewicht 


\begin{tabular}{|c|c|}
\hline $\mathrm{kg}$ & Kilogramm \\
\hline $\mathrm{kV}$ & Kilovolt \\
\hline$k \vee p$ & Kilo Voltage Peak \\
\hline $\mathrm{mA}$ & Milliampere \\
\hline $\mathrm{mg}$ & Milligramm \\
\hline Micro-CT & Micro-Computed Tomography \\
\hline $\min$ & Minute \\
\hline $\mathrm{ml}$ & Milliliter \\
\hline $\mathrm{mm}$ & Millimeter \\
\hline $\mathrm{mm}^{2}$ & Quadratmillimeter \\
\hline $\mathrm{mm}^{3}$ & Kubikmillimeter \\
\hline MMA & Methylmethacrylat \\
\hline MORE & Multiple Outcomes of Raloxifene Evaluation \\
\hline $\mathrm{ms}$ & Millisekunde \\
\hline MW & Mittelwert \\
\hline$\mu \mathrm{m}$ & Mikrometer \\
\hline$\mu$ Strain & Mikrostrain \\
\hline $\mathrm{N}$ & Newton \\
\hline $\mathrm{NIH}$ & National Institutes of Health \\
\hline $\mathrm{nm}$ & Nanometer \\
\hline OVX & Ovarektomie \\
\hline PTH & Parathormon \\
\hline s. & siehe \\
\hline SD & Standard Deviation \\
\hline SERMs & Selective Estrogen Receptor Modulators \\
\hline SHAM & Scheinoperation \\
\hline SOTI & Spinal Osteoporosis Therapeutic Intervention \\
\hline $\mathrm{TC}$ & Tetracyclin \\
\hline TROPOS & Treatment of Peripheral Osteoporosis \\
\hline UV & Ultraviolettstrahlung \\
\hline WBV & Whole-Body Vibration \\
\hline WHI & Women's Health Initiative \\
\hline
\end{tabular}


WHO

XO

z.B.

ZTE
World Health Organization

Xylenol-Orange

zum Beispiel

Zentrale Tierexperimentelle Einrichtung

(der Universitätsmedizin Göttingen) 


\section{$1 \quad$ Einleitung}

\subsection{Osteoporose}

\subsubsection{Definition}

"Osteoporose ist als Skeletterkrankung definiert, die durch eine unzureichende Knochenfestigkeit charakterisiert ist, welche zu einem erhöhten Frakturrisiko prädisponiert. Die Knochenfestigkeit spiegelt dabei primär das Zusammenwirken von Knochendichte und Knochenqualität wider" (NIH Consensus Statement 2000, Übersetzung aus Pfeilschifter 2006, S. 22). Deutlich pragmatischer als die zitierte "Consensus-Definition" ist die Osteoporose-Definition der Weltgesundheitsorganisation (WHO 1994). Nach ihr liegt eine Osteoporose dann vor, wenn die Knochendichte mehr als 2,5 Standardabweichungen (SD) unterhalb des Mittelwerts der Knochendichte skelettgesunder Erwachsener zwischen dem 20. und dem 40. Lebensjahr liegt. Diese gemessene Standardabweichung wird als T-Score bezeichnet. Grundlage der Knochendichtemessung ist dabei die duale Röntgenabsorptiometrie (Dual Energy X-ray Absorptiometry = DXA).

\subsubsection{Sozioökonomische Bedeutung}

Unabhängig von der gewählten Definition hat die Osteoporose in jedem Fall ein erhöhtes Frakturrisiko zur Folge. Die osteoporosebedingten Frakturen manifestieren sich vor allem an der Wirbelsäule, am proximalen Femur und am distalen Radius. Für die Betroffenen haben sie Schmerzen, Immobilität und nicht selten auch Invalidität zur Folge, auch eine frakturbedingt deutlich erhöhte Mortalität steht außer Frage (Johnell et al. 2004). Allein nach osteoporoseassoziierten Hüftfrakturen liegt die Einjahresmortalität zwischen 20 und 24 \% (Cooper et al. 1993; Leibson et al. 2002).

Von der Weltgesundheitsorganisation WHO wird die Osteoporose zu den zehn gravierendsten Erkrankungen überhaupt gezählt (Bartl $R$ und Bartl C 2008). Die 
sozioökonomische Dimension der Erkrankung wird durch Schätzungen verdeutlicht, nach denen weltweit rund 200 Millionen Menschen an Osteoporose und ihren Folgen leiden (Lane 2006). Man geht davon aus, dass 30-50 \% aller Frauen und 15-30 \% aller Männer im Laufe ihres Lebens zumindest einen osteoporosebedingten Knochenbruch erleiden (Randell et al. 1995). Weltweit sind im Jahr 2000 rund 9 Millionen osteoporoseassoziierte Frakturen aufgetreten, die meisten davon (34,8\%) wurden in Europa registriert (Johnell und Kanis 2006).

Allein in Deutschland waren im Jahr 2003 7,8 Millionen Patienten von der Osteoporose betroffen, 6,5 Millionen davon Frauen. Die hierzulande durch das Leiden verursachten Behandlungskosten werden auf 5,4 Milliarden Euro pro Jahr geschätzt (Häussler et al. 2007). Die steigende Lebenserwartung und veränderte Lebensgewohnheiten lassen für die Zukunft eine weitere Zunahme der Inzidenz der Erkrankung und der damit verbundenen Behandlungskosten erwarten. So geht ein Bericht der Europäischen Kommission aus dem Jahr 1998 von einer Zunahme der Inzidenz von Hüftfrakturen in Deutschland von 117.000 im Jahr 2000 auf 240.000 im Jahr 2040 aus (Häussler et al. 2007). Die europaweiten Folgekosten der Osteoporose werden nach Berechnungen von Kanis und Johnell (2005) von 31,7 Milliarden Euro im Jahr 2000 auf 76,7 Milliarden Euro im Jahr 2050 ansteigen.

\subsubsection{Klassifikation}

Traditionell wird die Osteoporose primär als Erkrankung postmenopausaler Frauen angesehen. Tatsächlich stellt die postmenopausale Osteoporose, die auch als Typ-1Osteoporose bezeichnet wird und sich 10-15 Jahre nach der Menopause manifestiert, die häufigste Form der Erkrankung dar. Rund 30 \% aller Frauen sind im entsprechenden Alter von ihr betroffen (Melton et al. 1993; Jones et al. 1994). Der aus der nachlassenden endokrinen Funktion der Ovarien resultierende Östrogenmangel führt dabei zu einem vermehrten Abbau des spongiösen Knochens, wovon insbesondere die Wirbelkörper und das proximale Femur betroffen sind. Deutlich seltener tritt die Typ-1-Osteoporose auch bei Männern auf, bei denen der spongiosabetonte Knochenverlust analog auf einen Testosteronmangel zurückgeführt wird (Stěpán et al. 1989). 
Mit Erreichen des 70. Lebensjahrs geht die Typ-1- oder postmenopausale Osteoporose definitionsgemäß in die Typ-2- oder senile Osteoporose über. Als Ursache dieser Form der Erkrankung werden neben anderen physiologischen Alterungsprozessen ein Mangel an Bewegung, Calcium und Vitamin D angenommen. Der Knochenmasseverlust ist dabei nicht mehr auf die Spongiosa beschränkt, sondern manifestiert sich zunehmend auch in der Kortikalis und damit an den Röhrenknochen der Extremitäten. Auch die senile Osteoporose betrifft bevorzugt Frauen im Verhältnis 2:1 (Bartl R und Bartl C 2008).

Im klinischen Alltag ist die Differenzierung zwischen Typ-1- und Typ-2-Osteoporose kaum relevant, beide Formen der Erkrankung werden zusammen mit der seltenen, idiopathischen juvenilen Osteoporose unter dem Begriff der idiopathischen oder primären Osteoporosen subsumiert. Ihnen gegenüberzustellen sind die sekundären Osteoporosen, bei denen sich der Knochenmasseverlust auf eine Grunderkrankung, auf Medikamente oder auf Immobilisation zurückführen lässt. Die zumeist laborchemische Identifizierung einer sekundären Osteoporose ist von klinischer Bedeutung, da sie in der Regel vordringlich eine Behandlung der Grunderkrankung erfordert. Die sekundären Formen machen jedoch nur $5 \%$ aller Osteoporosefälle aus (Bartl R und Bartl C 2008).

\subsubsection{Frakturheilung bei Osteoporose}

Pathognomonisch für die Osteoporose ist nicht nur das erhöhte Frakturrisiko, sondern auch eine deutliche Verzögerung und qualitative Beeinträchtigung der Frakturheilung. Im Tierexperiment wurde das vielfach belegt:

So konnten Lill und Mitarbeiter am Tibiaschaft eines Osteoporose-Schafsmodells eine erhebliche Verzögerung der Frakturheilung im Vergleich zur nichtovarektomierten Kontrollgruppe nachweisen (2003). Eine quantitative und qualitative Beeinträchtigung der Kallusbildung wurde auch am osteoporotischen Rattenmodell für den gesamten Verlauf der Frakturheilung belegt: Für die frühe Phase der Frakturheilung bis zum Ablauf der 3. postoperativen Woche konnten NamkungMatthai und Mitarbeiter am Femurschaft der ovarektomierten Ratte gegenüber der nichtovarektomierten Kontrollgruppe eine Verringerung von Kallusquerschnitt und 
-dichte nachweisen, außerdem im biomechanischen Test auch eine Beeinträchtigung der Kallusstabilität und -elastizität (2001). Für die mittlere und späte Phase der Frakturheilung zwischen 4. und 18. Woche zeigten Wang und Mitarbeiter am Tibiaschaft der ovarektomierten Ratte ebenfalls eine gegenüber der Kontrollgruppe verringerte Dichte des Kallus bei Verschlechterung seiner biomechanischen Eigenschaften (2005). Auch neuere Untersuchungen an einem metaphysären Frakturmodell der ovarektomierten Ratte fanden bei den osteoporotischen Tieren einen im Vergleich zur nichtovarektomierten Kontrollgruppe in seiner Ausdehnung reduzierten und in seiner Belastbarkeit beeinträchtigten Kallus (Komrakova et al. 2010; Stuermer et al. 2010 b).

Die Beeinträchtigung der Frakturheilung bei Osteoporose wird unter anderem auf eine Steigerung der Osteoklastenaktivität und auf eine Verzögerung der Mineralisationsvorgänge zurückgeführt. Das Risiko einer Pseudarthrose ist dadurch deutlich erhöht und die Osteosynthese wird beim osteoporotischen Patienten zur chirurgischen Herausforderung (Hobby und Lee 2013).

\subsection{Allgemeine Standards der Osteoporosetherapie}

\subsubsection{Basistherapie}

Als Basis einer medikamentösen Osteoporosetherapie empfiehlt der Dachverband Osteologie in seiner Leitlinie (Dachverband Osteologie e.V. 2009) eine Zufuhr von 1000 mg Kalzium täglich mit der Nahrung. Wenn diese Kalziumzufuhr über die Ernährung nicht erreicht werden kann, sollte eine Kalziumsupplementierung erfolgen.

Vitamin D, das die Kalziumresorption im Darm steigert, kann mit der Nahrung aufgenommen oder unter UV-Exposition der Haut im Körper synthetisiert werden. Grundsätzlich empfehlenswert sind deshalb eine orale Zufuhr über Fisch und Gemüse sowie eine regelmäßige Sonnenlichtexposition. Zumeist ist bei der Osteoporosetherapie zusätzlich eine orale Vitamin-D-Supplementierung mit „Gabe von 800-2000 IE Vitamin D pro Tag oder einer äquivalenten Menge an Vitamin D in 
größeren Einnahmeintervallen" erforderlich (Dachverband Osteologie e.V. 2009, S. 316).

Dawson-Hughes und Mitarbeiter konnten 1997 zeigen, dass die Kombinationstherapie aus Kalzium und Vitamin D bei über 65-jährigen Männern und Frauen den Knochenabbau senkt und zugleich das Risiko extravertebraler Frakturen reduziert.

\subsubsection{Sturzprävention}

Bei älteren Menschen sollte einer Reduktion des Sturzrisikos besondere Aufmerksamkeit gewidmet werden, da über $50 \%$ aller Hüftfrakturen auf ein Sturzereignis zurückzuführen sind (Woolf und Akesson 2003). Im Rahmen einer Optimierung des häuslichen Umfeldes sollten Stolperfallen, soweit möglich, beseitigt werden. Auch Hilfsmittel wie Gehhilfen und Hüftprotektoren sind geeignet, das Risiko einer sturzbedingten Fraktur zu reduzieren. Darüber hinaus sollte die Notwendigkeit einer Therapie mit sedierenden oder die orthostatische Regulation kompromittierenden Medikamenten bei älteren Patienten kritisch hinterfragt werden. $A b$ einem Alter von 70 Jahren wird eine jährliche Sturzanamnese empfohlen.

\subsubsection{Körperliches Training}

Neben einer ausreichenden Kalzium- und Vitamin-D-Zufuhr bzw. Supplementierung und gegebenenfalls spezifischen medikamentösen Therapie empfiehlt der Dachverband Osteologie in seiner Leitlinie eine konsequente Förderung der körperlichen Aktivität, die bei älteren Patienten das Risiko proximaler Femurfrakturen senkt (Pfeilschifter 2006). Ein regelmäßiges körperliches Training verbessert dabei sowohl den Gleichgewichtssinn als auch die Effektivität der neuromuskulären Reaktion, wodurch die Häufigkeit von Stürzen ebenso reduziert wird wie ihre traumatisierende Wirkung auf den Knochen (Melton und Riggs 1985). Darüber hinaus steht ein direkter Einfluss des körperlichen Trainings auf den Knochenstoffwechsel seit langem außer Frage (Smith und Gilligan 1991). 


\subsection{Medikamentöse Osteoporosetherapie}

\subsubsection{Hormonersatztherapie (HRT)}

Zusätzlich zu den genannten Basismaßnahmen werden zur Behandlung der Osteoporose seit Jahren spezielle Medikamente eingesetzt, die entweder den erkrankungsbedingten Knochenabbau behindern (antiresorptive Substanzen) oder aber die Knochenneubildung stimulieren (osteoanabole Substanzen):

Da der überwiegende Anteil der Osteoporosefälle bei Frauen auf einen Östrogenmangel zurückzuführen ist, stellt die Hormonsubstitution einen naheliegenden und früh genutzten therapeutischen Ansatz dar. Tatsächlich lindert die Östrogengabe nicht nur klimakterische Beschwerden, sondern hat auch einen deutlichen osteoanabolen Effekt (Spelsberg et al. 1999; Rossouw et al. 2002). Da die alleinige Östrogengabe die Entstehung von Endometriumkarzinomen begünstigt, ist eine zusätzliche Gestagensubstitution erforderlich, die diesem Risiko entgegenwirkt (Beral et al. 2005). Die in dieser Form etablierte Hormonersatztherapie (hormone replacement therapy, HRT) galt bis in die späten 90er Jahre als goldener Standard zur Behandlung und Prävention der postmenopausalen Osteoporose.

Zwischen 1993 und 1998 wurden die Folgen dieser Hormonersatztherapie im Rahmen einer randomisierten, placebokontrollierten Studie der "Womens Health Initiative" WHI an 161.809 postmenopausalen Frauen systematisch untersucht. Dabei konnte eine signifikante Senkung von Hüft- und Wirbelkörperfrakturen um ein Drittel durch die Östrogen-Gestagen-Kombinationstherapie nachgewiesen werden. Zugleich zeigte sich unter der Kombinationstherapie aber ein drastischer Anstieg des relativen Risikos, an Mammakarzinomen, Lungenembolien und koronarer Herzkrankheit zu erkranken (Rossouw et al. 2002). Unter der Östrogen-Monotherapie war dagegen ein deutlicher Anstieg von Schlaganfällen zu verzeichnen (Anderson et al. 2004), so dass beide Studienarme vorzeitig abgebrochen werden mussten.

2003 wurden die Ergebnisse einer Befragung von 1.084.110 Frauen im Alter von 50 bis 64 Jahren veröffentlicht, von denen etwa die Hälfte zum Zeitpunkt der Befragung 
oder zuvor Hormone eingenommen hatten. Wie schon die WHI-Studie belegte auch diese "Million Women Study" ein insbesondere durch die Kombinationstherapie erhöhtes Risiko, an einem Mammakarzinom zu erkranken oder zu versterben (Beral 2003). Auch ein unter Hormonersatztherapie gesteigertes Risiko für das Auftreten von Ovarialkarzinomen wurde im Rahmen der Studie nachgewiesen (Beral et al. 2007).

Die im Rahmen der WHI-Studie und der Million Women Study eindrucksvoll belegten Risiken der Hormonersatztherapie hatten Ende der 90er Jahre eine grundsätzliche Neubewertung dieser Therapieform zur Folge, die heute aufgrund ihres ungünstigen Nutzen-Risikoverhältnisses nur noch in Ausnahmefällen zur Behandlung der Osteoporose eingesetzt wird (Pfeilschifter 2006).

\subsubsection{Selektive Östrogen-Rezeptor-Modulatoren (SERMs)}

Als naheliegende Alternative zur Hormonersatztherapie rückten Ende der 90er Jahre die selektiven Östrogen-Rezeptor-Modulatoren (SERMs) in den Fokus des therapeutischen Interesses. Es handelt sich dabei um Substanzen, deren östrogenagonistische oder -antagonistische Wirkung sich auf einzelne Organsysteme beschränkt, indem sie z.B. einen östrogenen Effekt auf den Knochen und das Fettgewebe haben, nicht aber auf Mamma und Uterus. Die SERMs entfalten dabei ihre organselektive Wirkung durch direkte Interaktion mit den Östrogenrezeptoren $(E R) \alpha$ und $\beta$, die in unterschiedlichen, gewebsspezifischen Konzentrationen in den verschiedenen Körpergeweben anzutreffen sind, wobei zusätzliche Coregulatoren eine Rolle spielen (Dutertre und Smith 2000).

Prototyp und einziges zur Prävention und Therapie der postmenopausalen Osteoporose weltweit zugelassenes Präparat dieser Substanzgruppe ist das Raloxifen (Gennari et al. 2007), das die Knochendichte postmenopausaler Frauen innerhalb von 2 Jahren um 2-3\% verbessert, ohne dabei die Brustdrüse oder das Endometrium zu stimulieren (Ettinger et al. 1999). Unter einer Raloxifen-Behandlung scheint sich das Mammakarzinomrisiko sogar zu reduzieren, das Thromboembolierisiko entspricht allerdings dem einer Östrogentherapie (Cummings et al. 1999). Die internationale MORE-Studie (Multiple Outcomes of Raloxifene Evaluation) 
konnte zeigen, dass Raloxifen das Risiko für das Erstauftreten einer Wirbelkörperfraktur bei postmenopausalen Frauen halbiert, ohne allerdings die Inzidenz von extravertebralen Frakturen zu beeinflussen (Cummings et al. 1998; Ettinger et al. 1999).

\subsubsection{Bisphosphonate}

Die mit Abstand wichtigste Rolle in der Behandlung der Osteoporose spielen derzeit die seit 30 Jahren zu diesem Zweck in Deutschland zugelassenen Bisphosphonate. Es handelt sich dabei um synthetische Analoga des natürlich vorkommenden Pyrophosphats, die sich auf der Knochenoberfläche und dort vor allem in den Resorbtionslakunen anreichern und dabei durch Inhibition der Osteoklastenaktivität und Reaktivierung inaktiver Osteoblasten eine osteoprotektive Wirkung entfalten (Bartl R und Bartl C 2008). Zur Zeit kommen aus dieser antiresorptiven Substanzgruppe vor allem die Aminobisphosphonate Alendronat, Risedronat und Ibandronat zum Einsatz.

Bei postmenopausalen Frauen mit Osteoporose ist für Alendronat und Risedronat eine signifikante Senkung des Risikos für vertebrale und nichtvertebrale Frakturen um ca. 50 \% vielfach belegt (Black et al. 1996; Cummings et al. 1998; Harris et al. 1999; Pols et al. 1999; McClung et al. 2001). Auch lbandronat senkt das Risiko, eine vertebrale Fraktur zu erleiden um über 50 \% (Chesnut et al. 2004), ein Effekt auf das Risiko nichtvertebraler Frakturen ist für diese Substanz derzeit aber nicht nachzuweisen (Grey und Reid 2006).

Die Bisphosphonate gelten als die derzeit effektivsten Medikamente in der Behandlung aller Formen der Osteoporose, sowohl bei Frauen als auch bei Männern (Bartl R und Bartl C 2008).

\subsubsection{Parathormon}

Das in der Nebenschilddrüse synthetisierte Parathormon (PTH) setzt physiologischerweise Kalzium aus dem Knochen frei, um so die Serumkalziumkonzentration zu erhöhen. Bei kontinuierlicher Zufuhr führt es entsprechend zu 
Knochenverlust und Osteolysen (Canalis et al. 2007; Gao et al. 2008). Wird es dagegen unphysiologischerweise pulsatil verabreicht, so hat es einen deutlich osteoanabolen Effekt.

Neer und Mitarbeiter konnten 2001 zeigen, dass das rekombinante humane Parathormonfragment Teriparatid (PTH (1-34)) bei postmenopausalen Osteoporosepatientinnen unter pulsatiler Applikation über 21 Monate die Knochendichte am Schenkelhals um $3 \%$ und an der Wirbelsäule um $9 \%$ steigert und die Anzahl neu aufgetretener Wirbelfrakturen um $65 \%$ senkt.

Zurzeit sind die Parathormonfragmente $\mathrm{PTH}(1-34)$ und $\mathrm{PTH}(1-84)$ zur Behandlung der postmenopausalen Osteoporose mit hohem bis sehr hohem Frakturrisiko zugelassen.

\subsubsection{Strontiumranelat}

Ein relativ neues Präparat zur Behandlung der postmenopausalen Osteoporose ist das seit 2004 auf dem deutschen Markt zugelassene Strontiumranelat. Es handelt sich dabei um das knochenaffine Element Strontium aus der Gruppe der Erdalkalimetalle, das zur Verbesserung der Resorption und Verträglichkeit an Ranelicsäure gebunden ist. Strontiumranelat wirkt osteoanabol durch Steigerung der Präosteoblastenreplikation und der Kollagensynthese, zudem auch antiresorptiv durch Hemmung der Osteoklasten und ihrer Vorläuferzellen (Reginster et al. 2005).

Im Rahmen der SOTI-Studie (Spinal Osteoporosis Therapeutic Intervention) konnte 2004 gezeigt werden, dass die tägliche Einnahme von 2 g Strontium-Ranelat über 3 Jahre bei postmenopausalen Osteoporosepatientinnen zu einem Anstieg der Knochendichte am Schenkelhals um 8,3 \% und an der Wirbelsäule um 14,4 \% führt und zugleich das Risiko für des Erstauftreten einer Wirbelfraktur um $49 \%$ reduziert (Meunier et al. 2004).

In derselben Dosierung senkt Strontiumranelat innerhalb von 5 Jahren auch das Risiko für nichtvertebrale Frakturen um $15 \%$, wie die TROPOS-Studie (Treatment of Peripheral Osteoporosis) 2008 belegen konnte (Reginster et al. 2008). 


\subsection{Vibration}

\subsubsection{Wolffs Gesetz und das Utah-Paradigma}

Auf der Suche nach nichtmedikamentösen Behandlungsoptionen für die Osteoporose geriet in den letzen zwei Jahrzehnten die mechanische Knochenstimulation durch Vibration in den Fokus des wissenschaftlichen Interesses.

In seinem "Gesetz der Transformation der Knochen" hatte Julius Wolff schon 1892 die Anpassung des Knochengewebes an mechanische Druck- und Zugbelastungen beschrieben. Wolff hatte erkannt, dass der Knochen durch Modifikation seiner Trabekelstruktur mit geringstmöglicher Knochenmasse eine höchstmögliche mechanische Stabilität zu erreichen versucht. Das Wolff'sche Gesetz wurde weiterentwickelt und maßgeblich ergänzt durch die Arbeiten des amerikanischen Orthopäden und Chirurgen Harold M. Frost, der erstmals 1960 sein "Utah Paradigm of Skeletal Physiology" formulierte.

Frost beschreibt darin die kontinuierliche Anpassung von Knochenmasse und -festigkeit an die auf den Knochen einwirkenden Maximalkräfte als Regelkreis, für den er den Begriff "Mechanostat" prägte. Als Ursache für Knochenwachstum und Knochenabbau erkannte er dabei insbesondere die auf den Knochen einwirkenden, kurzzeitigen Maximalkräfte, die eine reversible Verformung des Knochens ("strain") bewirken. Das Maß für die Verformung des Knochens definierte er als $\mu$ Strain, wobei $1000 \mu$ Strain einer kraftinduzierten Längenänderung von 0,1 \% entsprechen. Für die meisten Tierarten variiert die Strainintensität zwischen unter $1000 \mu$ Strain beim Gehen bis zu $3200 \mu$ Strain bei anstrengenden Aktivitäten, bei galoppierenden Rennpferden wurden bis zu $5000 \mu$ Strain gemessen (Ehrlich und Lanyon 2002). An der menschlichen Tibia sind Strainintensitäten zwischen 800 und $1500 \mu$ Strain für eine ausgeglichene Knochenbilanz erforderlich. Unterhalb dieses Bereichs ist eine negative Knochenbilanz mit Abbau von Knochenmasse zu erwarten, bei Überschreiten von $1500 \mu$ Strain dagegen eine positive Bilanz mit Zunahme der Knochenmasse. Wird schließlich die Belastungsgrenze von $15000 \mu$ Strain überschritten, so ist mit einer Fraktur der Tibia zu rechnen (Frost 1960; Frost 2000). 
Vor dem Hintergrund dieser Erkenntnisse lag es nahe, eine mechanische Stimulation des Knochens zum Aufbau von Knochenmasse einzusetzen. Als einfaches Verfahren für eine derartige mechanische Stimulation des Knochens steht seit den neunziger Jahren die Ganzkörpervibration (Whole-Body Vibration, WBV) zur Verfügung (Fritton et al. 1997; Rubin et al. 2006).

\subsubsection{Ganzkörpervibration (WBV)}

Das Ganzkörpervibrationstraining war ursprünglich zur Behandlung der Muskelatrophie russischer Kosmonauten entwickelt worden und wurde später auch zur Muskelkräftigung bei Sportlern eingesetzt (Nazarov und Spivak 1985). In den neunziger Jahren wurden dann mobile Vibrationsplattformen zur vertikalen Ganzkörpervibration entwickelt, von denen man sich neben einer Stimulation der Muskulatur durch vibrationsinduzierte, reflektorische Verkürzung des Muskels (Delank und Gehlen 2006) speziell auch eine osteoanabole Wirkung durch elastische Verformung des Knochens erwartete (Fritton et al. 1997; Rubin et al. 2006). Mit den mobilen Geräten konnte die Wirksamkeit der Vibrationsbehandlung als nichtmedikamentöse, osteoanabole Therapieoption in tierexperimentellen Untersuchungen an verschiedenen Spezies nachgewiesen werden:

Xie und Mitarbeiter (2006) untersuchten den Einfluss der Ganzkörpervibration, indem sie 8 Wochen alte Mäuse über 3 Wochen täglich für 45 Minuten einer Vibration von $45 \mathrm{~Hz}$ aussetzten. Sie konnten dabei eine im Vergleich zur Kontrollgruppe um $30 \%$ gesteigerte Knochenbildungsrate bei entsprechend reduzierter Osteoklastenaktivität nachweisen. In einer zweiten Untersuchung führten sie an ausgewachsenen Mäusen über 6 Wochen täglich für 15 Minuten eine Vibrationsbehandlung mit $45 \mathrm{~Hz}$ durch, wodurch sich an der Tibia eine Zunahme des trabekulären Knochenvolumens um $14 \%$ erzielen ließ (Xie et al. 2008).

Rubinacci und Mitarbeiter (2008) vibrierten ovarektomierte Ratten über 8 Wochen jeweils 5 Tage für 20 Minuten und konnten dadurch eine signifikante Knochendichtezunahme erreichen. Bei der nichtovarektomierten Kontrollgruppe hatte die Vibrationsbehandlung keinen Effekt, woraus die Autoren auf eine Sensibilisierung des Knochens gegenüber der Vibration durch den Östrogenmangel schlossen. 
Sehmisch und Mitarbeiter (2009) beobachteten dagegen bei ovarektomierten und nichtovarektomierten Ratten gleichermaßen eine Zunahme der Knochendichte durch Vibration mit $90 \mathrm{~Hz}$ über 35 Tage.

Goodship und Mitarbeiter (2009) konnten an der osteotomierten Tibia von Schafen unter einer 10-wöchigen Vibrationsbehandlung mit jeweils 5 Behandlungen à 17 Minuten und einer Frequenz von $30 \mathrm{~Hz}$ einen gegenüber der nichtstimulierten Kontrollgruppe um 29 \% verstärkten Kallus und einen um 52 \% erhöhten Knochenmineralgehalt nachweisen. Die Schafe waren allerdings nicht ovarektomiert, weshalb der von Goodship nachgewiesene osteoanabole Effekt der Vibrationsbehandlung nur bedingt auf die in qualitativer und quantitativer Hinsicht verschlechterte Frakturheilung bei Osteoporose übertragen werden kann.

Beck und Mitarbeiter (2006) behandelten in einer unkontrollierten klinischen Studie 5 postmenopausale Frauen über 12 Monate zweimal täglich für 10 Minuten mit einer Vibration von $30 \mathrm{~Hz}$ und konnten damit am proximalen Femur der nichtdominanten Seite eine signifikante Zunahme des Knochenmineralgehalts um 2,03 $\pm 0,33 \%$ erreichen. Auch Verschueren und Mitarbeiter (2004) konnten in einer randomisierten Studie an 70 postmenopausalen Frauen mit einem Vibrationstraining von $35-40 \mathrm{~Hz}$ eine gegenüber der Kontrollgruppe signifikant gesteigerte Knochendichte am proximalen Femur nachweisen.

\subsubsection{Fragestellung und Zielsetzung}

Der derzeitige Stand der Wissenschaft lässt erwarten, dass die mechanische Stimulation des Knochens durch vertikale Ganzkörpervibration eine aussichtsreiche, nichtmedikamentöse Therapieoption für die Osteoporose darstellt. Vielfältige Unterschiede in den Studiendesigns hinsichtlich der eingesetzten Knochenanalysemethodik sowie insbesondere auch hinsichtlich Frequenz, Amplitude und Dauer der Vibration erschweren aber einen Vergleich der Studien.

Der osteoanabole Effekt der Vibration konnte schon für niedrige Amplituden nachgewiesen werden (Eisman 2001) und scheint nicht primär von der Amplitude abzuhängen (Rubin et al. 2006). Eine Abhängigkeit dieses Effekts von der 
Vibrationsfrequenz ist dagegen gut vorstellbar und naheliegend. Die vorliegende Arbeit soll daher klären, ob bestimmte Vibrationsfrequenzen zur mechanischen Stimulation des osteoporotischen Knochens geeigneter sind als andere.

Insbesondere soll dabei auch die Wirksamkeit einer Vibrationsbehandlung unterschiedlicher Frequenzen auf die Frakturheilung bei Osteoporose untersucht werden, die sich wie schon dargelegt (s. 1.1.4) von der normalen Frakturheilung in qualitativer und quantitativer Hinsicht erheblich unterscheidet. Der Effekt einer Vibrationsbehandlung auf den intakten Knochen osteoporotischer Tiere ist ebenso belegt (Rubinacci 2008; Sehmisch 2009) wie der auf die Frakturheilung nichtosteoporotischer Tiere (Goodship 2009). Ob die Vibrationsbehandlung auch in der Lage ist, die verschlechterte Frakturheilung bei Osteoporose zu beeinflussen, wurde bisher aber von anderen Arbeitsgruppen nicht untersucht. Ziel dieser Arbeit ist es daher insbesondere auch zu klären, ob sich die Frakturheilung bei Osteoporose durch eine Vibrationsbehandlung verbessern lässt und welche Frequenzen dazu gegebenenfalls besonders geeignet sind.

Um die vorangehend formulierten Fragen experimentell zu beantworten, soll mit dieser Arbeit die Frakturheilung an einem osteoporotischen Tiermodell unter einer vertikalen Vibrationsbehandlung mit Frequenzen von 35, 50, 70 und $90 \mathrm{~Hz}$ vergleichend untersucht werden.

Die ovarektomierte Ratte gilt als etabliertes Tiermodell für die postmenopausale Osteoporose (Kalu 1991; Wronski und Yen 1991; Frost und Jee 1992) und wird auch in den Richtlinien der amerikanischen Food and Drug Administration für erste Studien zur Prävention und Therapie der Osteoporose empfohlen (Thompson et al. 1995). Die proximale Tibiametaphyse der ovarektomierten Ratte gehört zu den Skelettarealen, an denen sich der östrogenmangelinduzierte, primär spongiöse Knochensubstanzverlust vorrangig manifestiert - bereits 3 Monate nach Ovarektomie ist hier ein Knochenmasseverlust von 50 \% zu erwarten (Wronski et al. 1985) - und gilt deshalb für Studien zur Wirksamkeit potentieller Osteoporosetherapeutika als besonders geeignet (Thompson et al. 1995). Trotzdem wurden Studien am osteoporotischen Rattenmodell bisher vorwiegend an den Diaphysen der Rattentibia (Nakajima et al 2002; Xu et al. 2004; Melhus et al. 2007) bzw. des Rattenfemurs 
(Kubo et al. 1999; Cao et al. 2002; Xu et al. 2003; Shi et al. 2010) durchgeführt. In der vorliegenden Arbeit soll die Frakturheilung der osteoporotischen Ratte dagegen an der Tibiametaphyse untersucht werden, an der sich die Osteoporose auch tatsächlich manifestiert.

Um die osteoanabole Potenz unterschiedlicher Vibrationsfrequenzen zu vergleichen und um zu klären, ob bestimmte Vibrationsfrequenzen zur Verbesserung der Frakturheilung bei Osteoporose geeigneter sind als andere, soll in dieser Arbeit die Frakturheilung an der proximalen Tibiametaphyse der ovarektomierten Ratte unter einer vertikalen Vibrationsbehandlung mit Frequenzen von 35, 50, 70 und $90 \mathrm{~Hz}$ untersucht werden. Die Frakturheilung soll dabei anhand eines biomechanischen Belastungstests qualitativ bewertet werden. Eine quantitative Bewertung der Frakturheilung soll primär mit Hilfe der Mikroradiographie erfolgen, da histologische Untersuchungsverfahren als Goldstandard der strukturellen Knochenanalytik gelten (Particelli et al. 2012). Ergänzend soll zur quantitativen Analyse auch die MicroComputertomographie (Micro-CT) herangezogen werden, die als verhältnismäßig neues Untersuchungsverfahren von unserer Arbeitsgruppe hier erstmals eingesetzt wird. Eine polychrome Sequenzmarkierung soll schließlich eine chronologische Analyse mit Aussagen zum zeitlichen Ablauf der Knochenkonsolidierung ermöglichen. 


\section{Material und Methoden}

\section{$2.1 \quad$ Versuchsaufbau}

\subsubsection{Zeitlicher Ablauf}

Der Versuch erstreckte sich insgesamt über 91 Tage. Zu Beginn wurden 90 weibliche Ratten randomisiert in sechs Gruppe von je 15 Tieren aufgeteilt. Die Ratten der Gruppen 2 bis 6 wurden ovarektomiert mit dem Ziel, die Entwicklung einer Osteoporose zu induzieren. Die Ratten der Gruppe 1 wurden dagegen als Kontrollkollektiv scheinoperiert (SHAM, siehe Tab. 1). Zwei der Tier starben bei der Ovarektomie, so dass das Kollektiv von diesem Zeitpunkt an aus lediglich 88 Ratten bestand.

Nach acht Wochen wurde bei allen Tieren eine Osteotomie und Osteosynthese beider proximaler Tibiametaphysen durchgeführt. 61 Tage nach Ovarektomie beziehungsweise SHAM-Operation wurde mit der mechanischen Stimulation durch die Vibrationsbehandlung begonnen, indem die Ratten täglich über 15 Minuten einer vertikalen Ganzkörpervibration (WBV) mit der ihrer Gruppe zugeordneten Frequenz ausgesetzt wurden. 15 der ovarektomierten Ratten wurden als OVX-Gruppe von der Vibrationsbehandlung ausgeschlossen. Auch die Tiere der nichtovarektomierten SHAM-Gruppe wurden nicht vibriert.

Tabelle 1: Gruppenzuordnung der Tiere

\begin{tabular}{|c|c|c|}
\hline \hline Tiernummer & Käfignummer & Behandlung \\
\hline \hline $1-15$ & $1-3$ & SHAM \\
\hline $16-30$ & $4-6$ & OVX \\
\hline $31-45$ & $7-9$ & OVX + WBV $35 \mathrm{~Hz}$ \\
\hline $46-59$ & $10-12$ & OVX + WBV $50 \mathrm{~Hz}$ \\
\hline $60-73$ & $13-15$ & OVX + WBV $70 \mathrm{~Hz}$ \\
\hline $74-88$ & $16-18$ & OVX + WBV $90 \mathrm{~Hz}$ \\
\hline
\end{tabular}

SHAM = Scheinoperation, $\quad$ OVX = Ovarektomie, $\quad$ WBV = Ganzkörpervibration 
Um spätere Rückschlüsse auf den zeitlichen Ablauf des Knochenumbaus unter der Vibrationsbehandlung zu ermöglichen, wurde während des Vibrationszeitraumes eine polychrome Sequenzmarkierung nach Rahn durchgeführt (Rahn 1976). Zu diesem Zweck wurden den Tieren am 13. Tag nach Osteotomie $90 \mathrm{mg} / \mathrm{kg} \mathrm{KG}$ Xylenol-Orange (XO) subkutan injiziert, am 18. Tag $10 \mathrm{mg} / \mathrm{kg} \mathrm{KG}$ Calcein-Grün (CG), am 24. und am 26. Tag jeweils $30 \mathrm{mg} / \mathrm{kg} \mathrm{KG} \mathrm{Alizarin-Komplexon} \mathrm{(AK)} \mathrm{sowie} \mathrm{am} 35$. Tag 30 mg/kg KG Tetracyclin (TC). Am selben Tag, also 35 Tage nach Osteotomie bzw. 91 Tage nach Versuchsbeginn, wurden die Tiere mit $\mathrm{CO}_{2}$ narkotisiert und durch Dekapitation getötet.

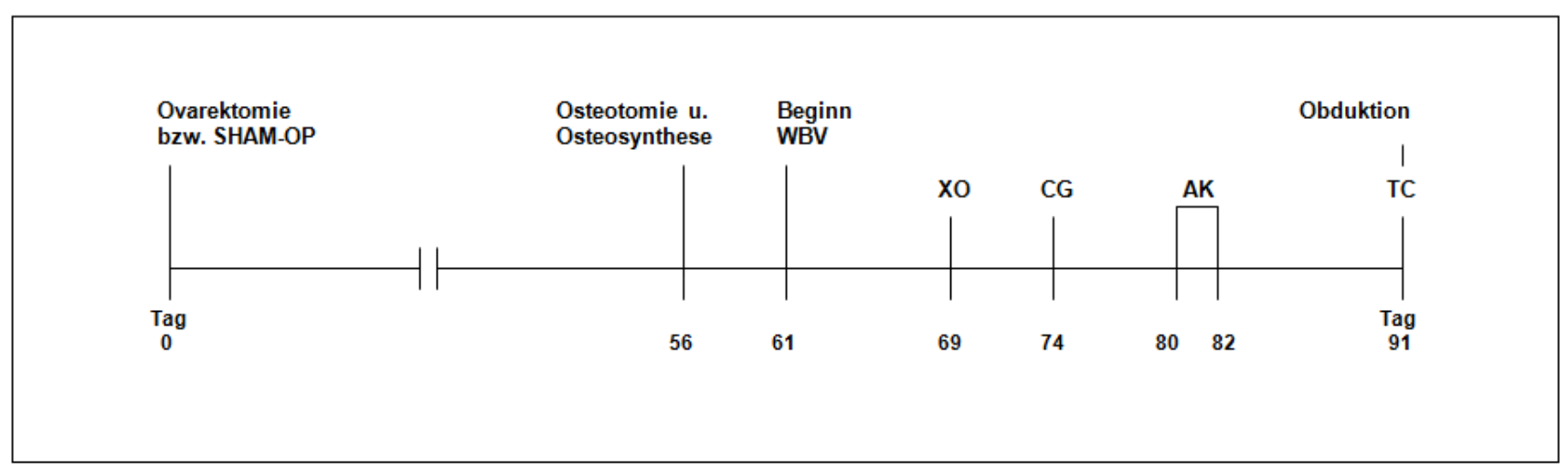

Abbildung 1: Zeitlicher Versuchsablauf. WBV=Ganzkörpervibration, XO=Xylenol-Orange, CG=Calcein-Grün, AK=Alizarin-Komplexon, TC=Tetracyclin

\subsubsection{Versuchstiere und Versuchstierhaltung}

Die Versuche wurden mit 90 weiblichen Sprague-Dawley-Ratten der Firma Harlan Laboratories, Indianapolis, durchgeführt. Über den gesamten Versuchszeitraum wurden die Ratten in der Zentalen Tierexperimentellen Einrichtung (ZTE) der Universitätsmedizin Göttingen gehalten, wobei durch das dort angestellte Fachpersonal aus Tierpflegerinnen und -pflegern unter Aufsicht von Dr. Schunck (Tierarzt ZTE) eine artgerechte Haltung gewährleistet wurde. 
Die Haltung der Ratten erfolgte in Käfigen vom Typ Makrolon ${ }^{\circledR}$ IV, die mit jeweils vier bis fünf Tieren besetzt wurden. Alle drei Tage wurden die Tiere in desinfizierte Austauschkäfige umgesetzt. Die Umgebungstemperatur betrug konstant $20{ }^{\circ} \mathrm{C}$ bei einer relativen Luftfeuchtigkeit von 55 \%. Zur Imitation eines möglichst natürlichen Tagesrhythmus wurde eine alternierende Hell-Dunkel-Periodik von jeweils 12 Stunden eingehalten. Futter wurde in Gestalt $10 \mathrm{~mm}$ großer Pellets (ssniff SM R/M, ssniff Spezialitäten $\mathrm{GmbH}$, Soest, Deutschland) ebenso wie Wasser ad libitum bereitgestellt. Aus dem am Ende einer jeden Woche für jede Gruppe protokollierten Futterverbrauch wurde der durchschnittliche Tagesverbrauch der Tiere berechnet. Am Anfang jeder Woche wurden die Ratten gewogen. Das durchschnittliche Körpergewicht (KG) der Tiere betrug zum Zeitpunkt der Ovarektomie $239 \mathrm{~g}$, wobei die leichteste Ratte $215 \mathrm{~g}$ und die schwerste $280 \mathrm{~g}$ wog.

Die hier beschriebenen Versuche waren von der Bezirksregierung Braunschweig unter dem Aktenzeichen AZ 33.9.42502-04/011/07 genehmigt worden.

\subsubsection{Ovarektomie}

Zur Durchführung der Ovarektomie wurden die Ratten zunächst mit $\mathrm{CO}_{2}$ sediert und dann gewogen. Die Anästhesie erfolgte anschließend durch intraperitoneale Applikation eines Gemisches aus Ketamin (Hostaket ${ }^{\circledR}$, Hoechst, Bad Soden, Deutschland) und Xylazin (Rompun®, Bayer, Leverkusen, Deutschland) im Verhältnis 5:3, von dem jeweils 0,01 $\mathrm{ml} / \mathrm{g} \mathrm{KG}$ verabreicht wurde.

Die Hautpartie distal des Rippenbogens wurde anschließend beidseits rasiert und desinfiziert. Durch einen paravertebralen Hautschnitt wurde bis zum Peritoneum in die Tiefe präpariert und die Bauchhöhle eröffnet. Nach Darstellung der Adnexe wurde die Tuba uterina ligiert und das Ovar abgesetzt. Der Wundverschluss erfolgte durch Vicryl-Naht des Peritoneums (Ethicon, Johnson \& Johnson, Norderstedt, Deutschland) und Klammern der Haut (Michel wound brackets, 12 × 3 mm, Gebrüder Martin $\mathrm{GmbH}$ \& Co.KG, Tuttlingen). Im Anschluss daran wurde auf der kontralateralen Seite in gleicher Weise verfahren.

Um eine einfache Identifizierung der einzelnen Tiere im weiteren Versuchsablauf zu ermöglichen, wurde jeder Ratte ein Transponder (Uno Micro-Id-System, Iso- 
Transponder 12 mm, UNO Roestvaststaal BV, Zevenaar, Niederlande) mit einem Injektor ins subkutane Nackenfettgewebe injiziert.

Zum Abschluss des Eingriffs wurden jedem Tier $3 \mathrm{ml}$ isotone Kochsalzlösung subkutan injiziert, um einem postoperativen Volumenmangel entgegenzuwirken. Die Tiere wurden dann in einen mit Zellstoff ausgekleideten Käfig gelegt und bis zum Erwachen beaufsichtigt.

\subsubsection{Osteotomie und Osteosynthese}

Acht Wochen nach der Ovarektomie wurde eine Osteotomie und Osteosynthese beider Tibiae durchgeführt. Die Anästhesie erfolgte dabei wie unter 2.1 .3 beschrieben. Nach Rasieren und Desinfizieren beider Hinterläufe wurde die Haut über der ventromedialen Tibia auf einer Länge von $3 \mathrm{~cm}$ eröffnet und die Streckmuskulatur unter Schonung des Periosts scharf abgesetzt. Mithilfe einer Schablone wurde die Osteotomiehöhe exakt $7 \mathrm{~mm}$ unterhalb des Kniegelenkspaltes markiert. Anschließend wurde eine 5-Loch-T-Platte nach Leibinger aus Titan (5705140 XS-Titanfixationsplatte T-Form 90, Stryker Trauma, Selzach, Schweiz) über der medialen Tibia so ausgerichtet, dass die beiden oberen Plattenlöcher in Höhe der Epiphyse lagen. Zunächst wurden dann der proximal-cephale und der am weitesten distal gelegene Schraubenkanal vorgebohrt und provisorisch mit je einer Schraube besetzt. Der proximal-kaudale und der zweite distale Schraubenkanal wurden lediglich vorgebohrt, in Höhe des verbleibenden Plattenlochs über der geplanten Osteotomielinie wurde keine Bohrung durchgeführt.

Nach temporärer Entfernung des Osteosynthesematerials wurde die Tibiaosteotomie in Höhe der Markierung unter Schonung der umgebenden Weichteile mit gepulstem Ultraschall (OT 7 Piezosurgery ${ }^{\circledR}$, Mectron Medical Technology, Carasco, Italien) senkrecht zur Längsachse der Tibia durchgeführt. Anschließend wurde die Titanplatte refixiert, wobei die proximalen Bohrkanäle mit zwei $7 \mathrm{~mm}$ 1.1er Schrauben besetzt wurden, die distalen Kanäle dagegen mit jeweils einer $5 \mathrm{~mm}$ und einer $4 \mathrm{~mm}$ 1.1er Schraube.

Durch das Festlegen der Plattenposition und Bohren der Schraubenkanäle vor Durchführung der Osteotomie entstand dabei jeweils ein standardisierter Osteotomiespalt von 0,5 mm entsprechend der Dicke des verwendeten Sägeblattes. 


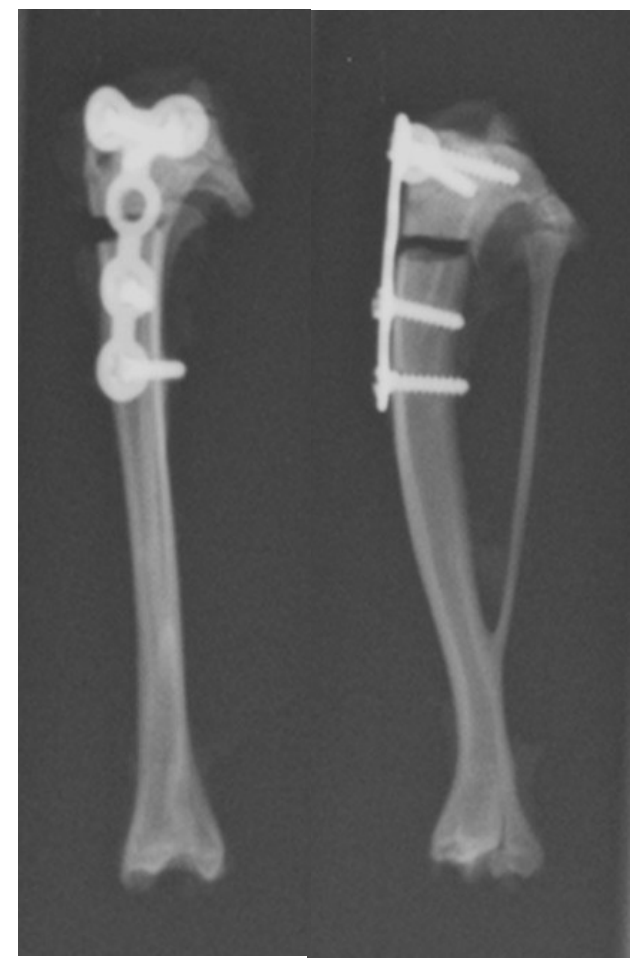

Abbildung 2:

Osteotomierte Rattentibia im

Röntgenbild ap und seitlich

mit Osteosyntheseplatte und

Schrauben

Nach Festziehen der Schrauben wurde die Streckmuskulatur reponiert und mit 4.0 Vicryl-Naht readaptiert. Der Hautverschluss erfolgte durch Klammern. Mit dem kontralateralen Hinterlauf wurde in gleicher Weise verfahren.

Nach Abschluss des Eingriffs wurde den Ratten zur Beruhigung einmalig Decentan ${ }$ (Merck, Darmstadt, Deutschland) in einer Dosierung von $5 \mathrm{mg} / \mathrm{kg} \mathrm{KG}$ subkutan verabreicht, zur Volumensubstitution außerdem $3 \mathrm{ml}$ isotone Kochsalzlösung. Wie schon nach der Ovarektomie wurden die Tiere dann in einen mit Zellstoff gepolsterten Käfig gelegt und bis zum Abklingen der Narkosewirkung überwacht.

Zur Analgesie erhielten die Ratten am OP-Tag und den folgenden zwei Tagen je 2mal Rimadyl ${ }^{\circledR}$ (Pfizer, Karlsruhe, Deutschland) in einer Dosierung von $4 \mathrm{mg} / \mathrm{kg} \mathrm{KG}$ subkutan.

Zur räumlichen Orientierung wird im nachfolgenden Text der Begriff „Plattenebene“ verwendet, der sich jeweils auf die Auflagefläche der 5-Loch-T-Platte auf der ventromedialen Tibia bezieht. Der Begriff „Schraubenebene“ bezeichnet dagegen die imaginäre Ebene, die durch alle Schraubenkanäle am Längsschenkel der T-Platte verläuft. Die beiden so definierten Ebenen stehen senkrecht aufeinander. 


\subsubsection{Vertikale Ganzkörpervibration}

5 Tage nach Osteotomie und Osteosynthese und damit 61 Tage nach der Ovarektomie wurde mit der vertikalen Ganzkörpervibration (whole-body vibration, WBV) begonnen. Die Tiere wurden von diesem Zeitpunkt an über insgesamt 30 Tage täglich jeweils nachmittags für 15 Minuten der Vibration ausgesetzt. Zum Einsatz kam dabei ein Vibrationstisch (Vibriertisch VTG, Drehstrom-Vibrationsmotor Typ HVL/HVE, Vibra Schultheis, Offenbach, Deutschland), auf dem ein aus dünnem Kunststoff-Polstermaterial bestehender Käfig befestigt war. Der Käfig wurde mit jeweils 7 bis 8 Tieren besetzt. Um eine Abschwächung des Vibrationseffekts zu vermeiden, wurde darauf geachtet, dass sich die Ratten nicht übereinander legten und jedes Tier stets Bodenkontakt hielt. Ansonsten konnten sich die Tiere während der Vibration frei im Käfig bewegen.

Die Ratten waren zu Versuchsbeginn randomisiert in 6 Gruppen von jeweils 14 bis 15 Tieren aufgeteilt worden. Die 4 zur Vibration vorgesehenen Gruppen wurden nun mit der gruppenspezifischen Frequenz von 35, 50, 70 oder 90 Hertz vibriert, um so potentiell unterschiedliche Effekte der verschiedenen Frequenzen zu erfassen (siehe Tab. 1). Die Amplitude der Vibration betrug dabei stets $0,5 \mathrm{~mm}$.

\subsubsection{Präparation der Tibiae}

Die Obduktion der Tiere erfolgte 14 Wochen nach der Ovarektomie und damit 6 Wochen nach der Osteosynthese, da die Frakturheilung zu diesem Zeitpunkt noch nicht vollständig abgeschlossen war und der zu untersuchende Kallus damit noch keinen peripheren Resorptionsvorgängen unterworfen war. Die Ratten wurden zu diesem Zweck mit $\mathrm{CO}_{2}$ narkotisiert und durch Dekapitation getötet. Das Blut wurde aufgefangen und für laborchemische Analysen aufbewahrt. Das Körpergewicht der Tiere lag zu diesem Zeitpunkt bei durchschnittlich $314 \mathrm{~g}$ (272 - $370 \mathrm{~g}$ ).

Eine der beiden randomisiert ausgewählten Tibiae wurde im Kniegelenk und im oberen Sprunggelenk exartikuliert und sorgfältig freipräpariert, wobei darauf geachtet wurde, den Verbund aus Tibia und Fibula intakt zu lassen und den Frakturkallus nicht zu beschädigen. Nach Entfernen des Osteosythesematerials wurde das Präparat in ein mit der entsprechenden Versuchstiernummer gekennzeichnetes Kunststoff- 
schraubgefäß gelegt und in flüssigem Stickstoff eingefroren, um dann bei $-20 \mathrm{C}^{\circ}$ gelagert zu werden.

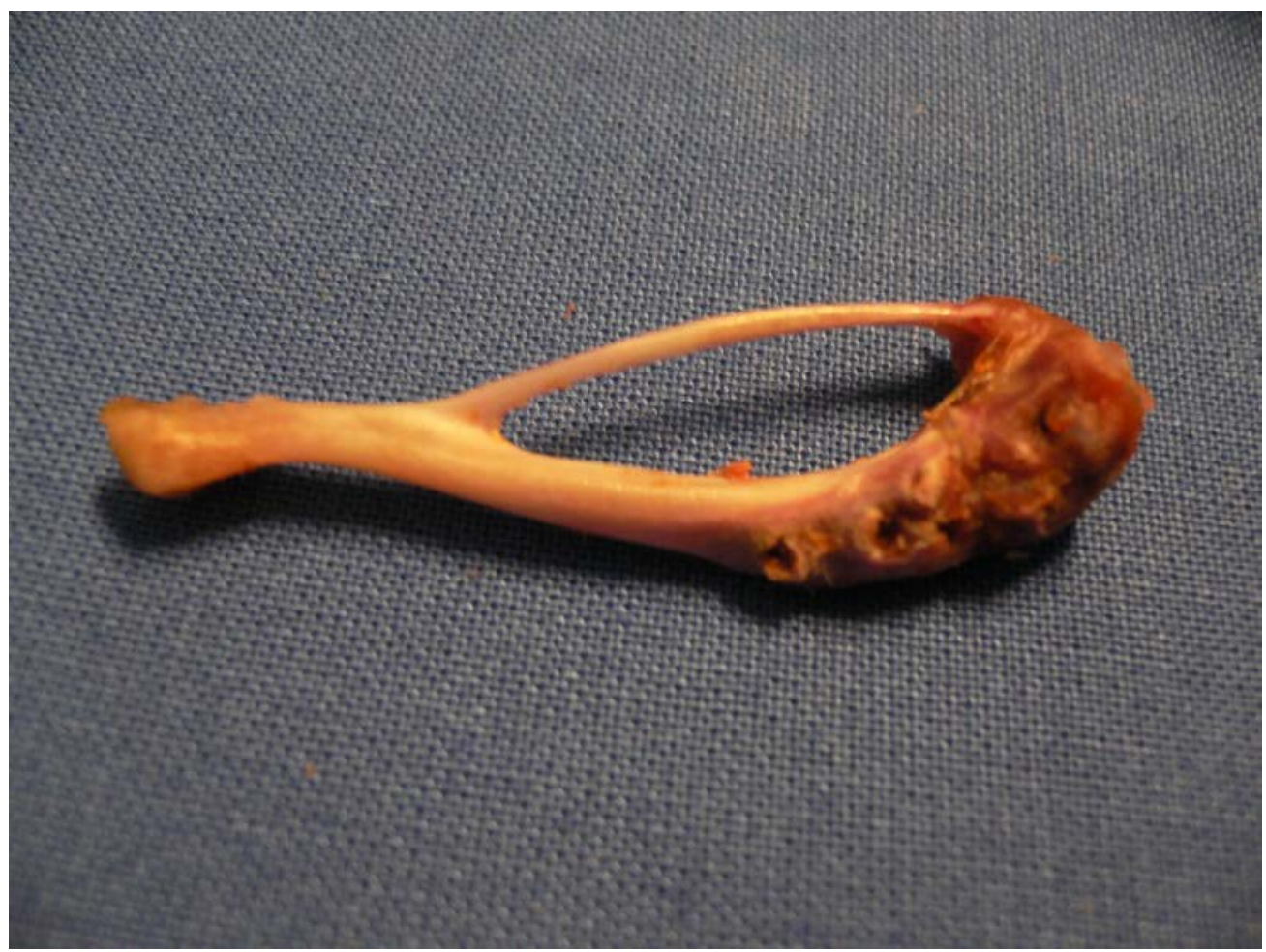

Abbildung 3: Tibia-Fibulapräparat

Die kontralaterale Tibia wurde ebenfalls freigelegt und vom Osteosynthesematerial befreit. Der kallustragende Knochenabschnitt wurde mit einer Zange isoliert und für spätere genanalytische Untersuchungen sofort in flüssigem Stickstoff bei $-80 \mathrm{C}^{\circ}$ eingefroren.

Im Anschluss wurde der Uterus entnommen und gewogen. Schließlich wurden auch die Femora, die Lendenwirbelkörper sowie Teile der Muskulatur entfernt und für weitere Versuche bereitgestellt. 


\subsection{Röntgenuntersuchung}

\subsubsection{Technische Durchführung der Röntgenuntersuchung}

Die Tibia-Fibulapräparate wurden anschließend vor Durchführung des biomechanischen Biegetests einer Röntgenuntersuchung unterzogen. Der gesamte TibiaFibula-Verbund wurde dabei in der Platten- und in der Schraubenebene und damit in zwei aufeinander senkrecht stehenden Ebenen geröntgt. Bei einer Röhrenspannung von $40 \mathrm{kV}$ und einer Stromstärke von 0,3 mA betrug die Belichtungszeit 6 Minuten. Die Untersuchung wurde mit einem Faxitron-Röntgengerät (Modell-Nr. 43855A, IL 60089, Faxitron X-ray System, Hewlett-Packard, San Diego, USA) durchgeführt unter Verwendung eines AGFA-Films (AGFA Structurix D4 DW ETE ISO 9001, AGFA Graphics Germany GmbH \& Co. KG, Düsseldorf, Deutschland).

\subsubsection{Kriterien der röntgenologischen Befundung}

Bei der Befundung der Röntgenaufnahmen wurde zunächst die Qualität der Tibiafrakturheilung begutachtet und eventuelle Pseudarthrosen wurden dokumentiert. Anschließend erfolgte eine Prüfung auf Integrität der Fibula, bei der eventuelle Fibulafrakturen oder -defekte ebenfalls protokolliert wurden. Die Bilder wurden schließlich auf das Vorliegen röntgenologischer Infektzeichen hin begutachtet.

Alle Präparate, bei denen eine der obenstehenden Auffälligkeiten festgestellt werden konnte, wurden vom weiteren Versuchsablauf ausgeschlossen. 


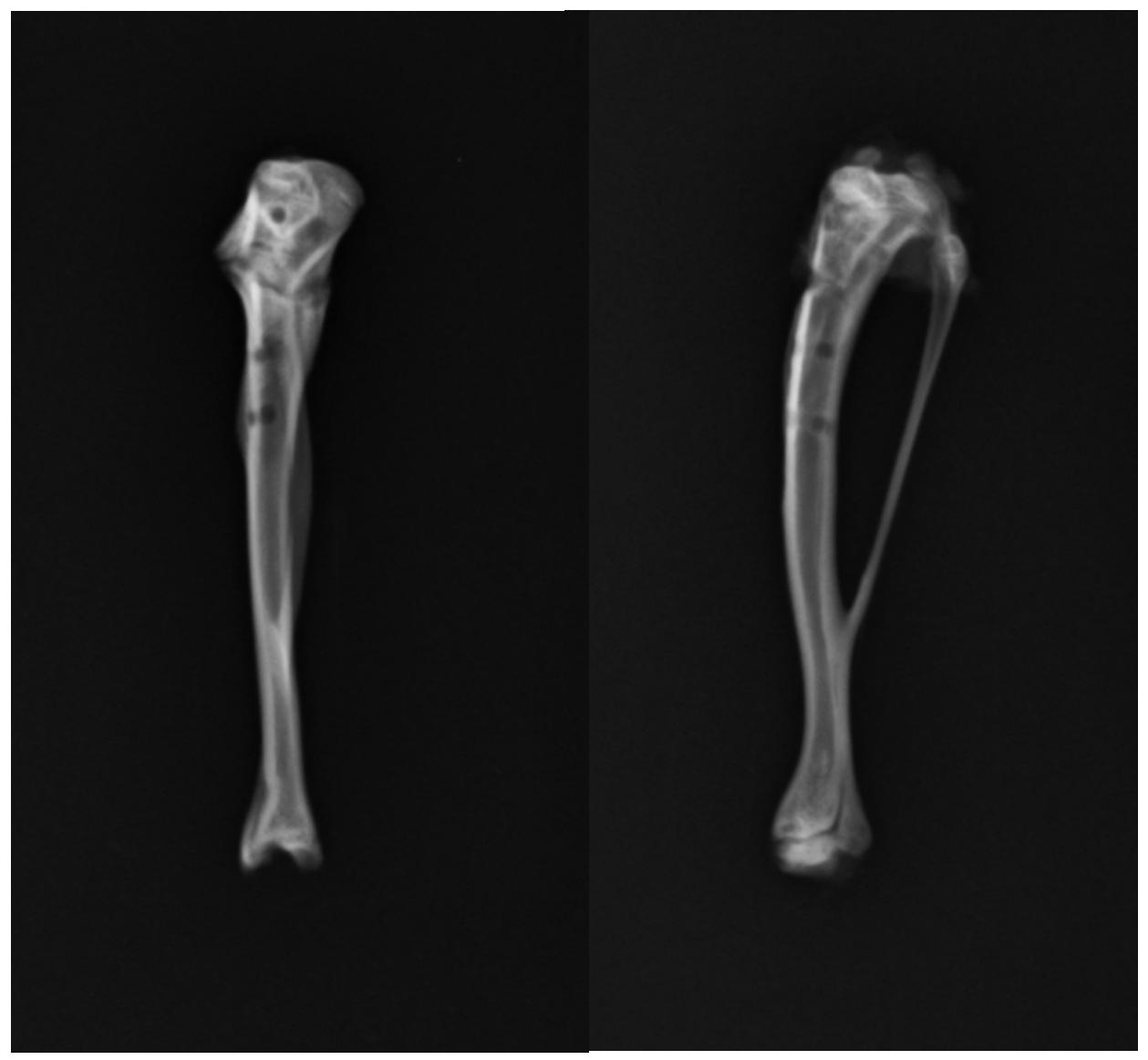

Abbildung 4: Röntgenbild einer Rattentibia ap und seitlich

\subsection{Biomechanischer Biegetest}

\subsubsection{Prinzip des biomechanischen Biegetests}

Vor Durchführung weiterer bildgebender Untersuchungen wurden die Tibiapräparate einem standardisierten Biegetest unterzogen, um ein biomechanisches Stabilitätsprofil des Frakturkallus mit Aussagen über dessen Elastizität und Bruchfestigkeit zu gewinnen. Bei dieser Untersuchung wurde mit Hilfe einer Werkstoffprüfmaschine eine senkrecht zur Längsachse wirkende Kraft auf die ventrale Tibiakante ausgeübt und die Durchbiegung des Knochens in Abhängigkeit von der auf inn einwirkenden Kraft gemessen. 
Dieser Test wurde vor der Micro-Computertomographie durchgeführt, um eine Veränderung der biomechanischen Eigenschaften des Knochens, zum Beispiel durch Austrocknung während der zeitaufwendigen CT-Untersuchung, zu vermeiden.

\subsubsection{Versuchsaufbau des biomechanischen Biegetests}

Vor Durchführung des Biegetests wurden die Tibiapräparate schonend aufgetaut, indem sie zunächst über 4 bis 6 Stunden im Kühlschrank bei $4{ }^{\circ} \mathrm{C}$ gelagert wurden und anschließend jeweils 10 Minuten bei Raumtemperatur. Es wurden dann zunächst die Fibulae der vollständig aufgetauten Präparate auf das Vorliegen von Frakturen untersucht, wobei ein Frakturnachweis den Ausschluss von weiteren Untersuchungen zur Folge hatte.

Um eine standardisierte, stabile Lagerung der Tibia für den Biegetest zu ermöglichen, wurde die Fibula anschließend mit einer Knochenzange behutsam entfernt. Die Stabilität der Tibiae wurde dann vorsichtig manuell geprüft, eventuelle Pseudarthrosen wurden dokumentiert und die entsprechenden Knochen ebenfalls von den weiteren Tests ausgeschlossen.

Alle anderen Tibiae wurden einzeln in einer für diesen Versuch entwickelten Bruchvorrichtung gelagert (Stuermer et al. 2006). Diese besteht aus einem $65 \times 25 \times$ $10 \mathrm{~mm}$ großen Aluminiumblock, der auf einer Aluminiumplatte fixiert ist (s. Abb. 5). Eine $3 \mathrm{~mm}$ breite, quer in den Block gefräste Nut ermöglicht eine stabile Lagerung der dorsalen Tibiakondylen, während eine mittig in Längsrichtung des Blocks verlaufende zweite Nut von $6 \mathrm{~mm}$ Breite der Aufnahme des distalen Tibiaendes dient. Vier vertikale Lagerungsbolzen in Form frei rotierender Aluminiumzylinder ermöglichen eine seitliche Abstützung des Präparates ohne dieses zu fixieren und damit in seinem Biegeverhalten zu beeinflussen. 


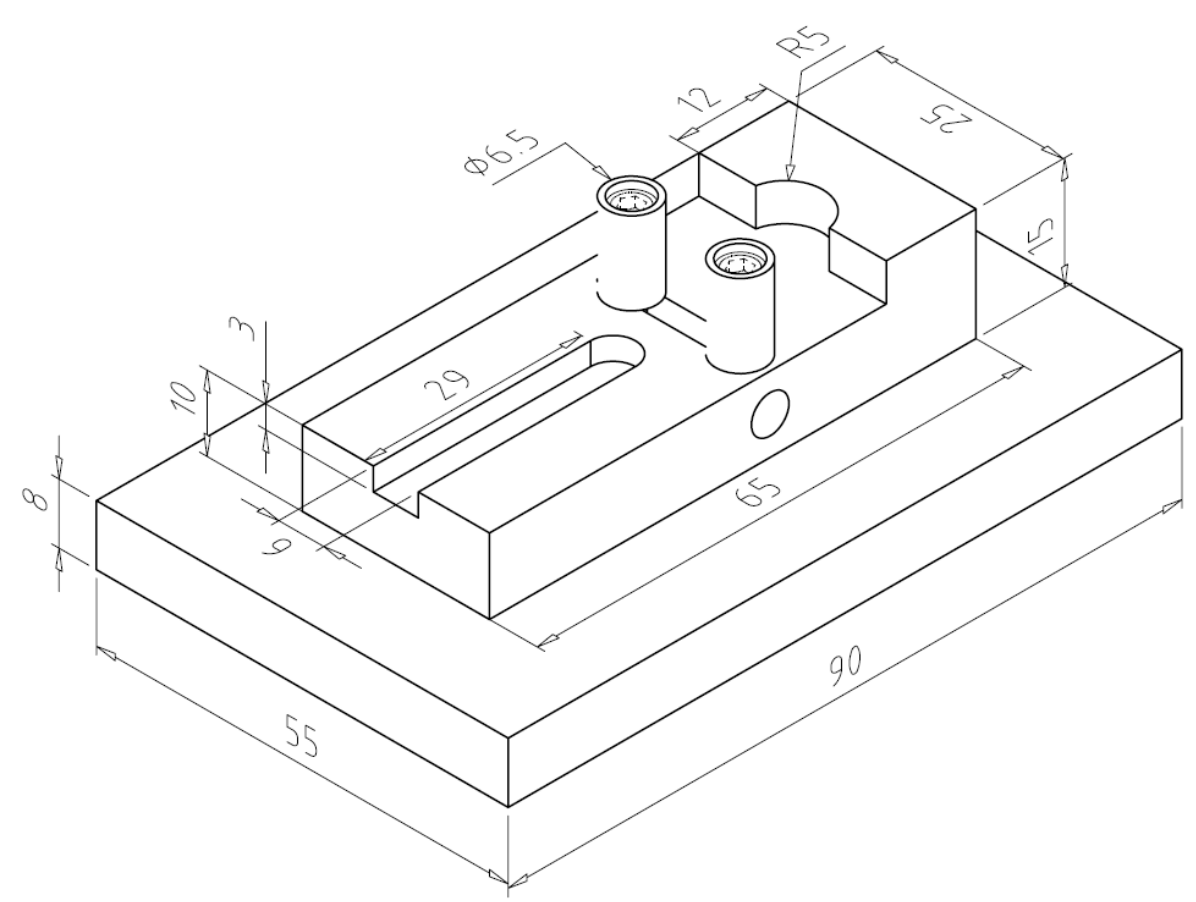

Abbildung 5: Lagerungsblock der Bruchvorrichtung, alle Angaben in mm

Der Lagerungsblock wurde dann mitsamt der Tibia in einer Werkstoffprüfmaschine platziert (Typ 145660 Z020/TND Zwick/Roell, Ulm, Deutschland). Durch die Werkstoffprüfmaschine wurde ein Druck auf die Vorderkante der Tibia ausgeübt, wobei die Kraftübertragung über einen Rollstempel mit zirkulärer Nut erfolgte (Stuermer et al. 2006). Dieser Stempel wurde $2 \mathrm{~mm}$ distal der Osteotomielinie platziert und auf die Tibiavorderkante abgesenkt, bis eine Vorkraft von $1 \mathrm{~N}$ erreicht war. Nach Überprüfen und erforderlichenfalls Korrigieren der korrekten Position von Rollenstempel und Präparat wurde der Kompressionstest gestartet.

Das „testXpert"-Programm (Zwick/Roell, Ulm, Deutschland) senkte dann den Rollenstempel mit einer Geschwindigkeit von $50 \mathrm{~mm} / \mathrm{min}$ weiter hinab, wodurch die Tibia zunehmend verbogen wurde. Die für eine Verbiegung von 0,1 mm Wegstrecke erforderliche Kraft wurde von der Software jeweils automatisch gemessen und als Kraft-Weg-Diagramm aufgezeichnet. Der Messbereich lag dabei zwischen 2 und 500 $\mathrm{N}$ bei einer relativen Genauigkeit von 0,2-0,4\%. Bei Erreichen der Maximalkraft wurde der Test von der Software automatisch abgebrochen, so dass eine Fraktur des Kallus in allen Fällen vermieden werden konnte. 


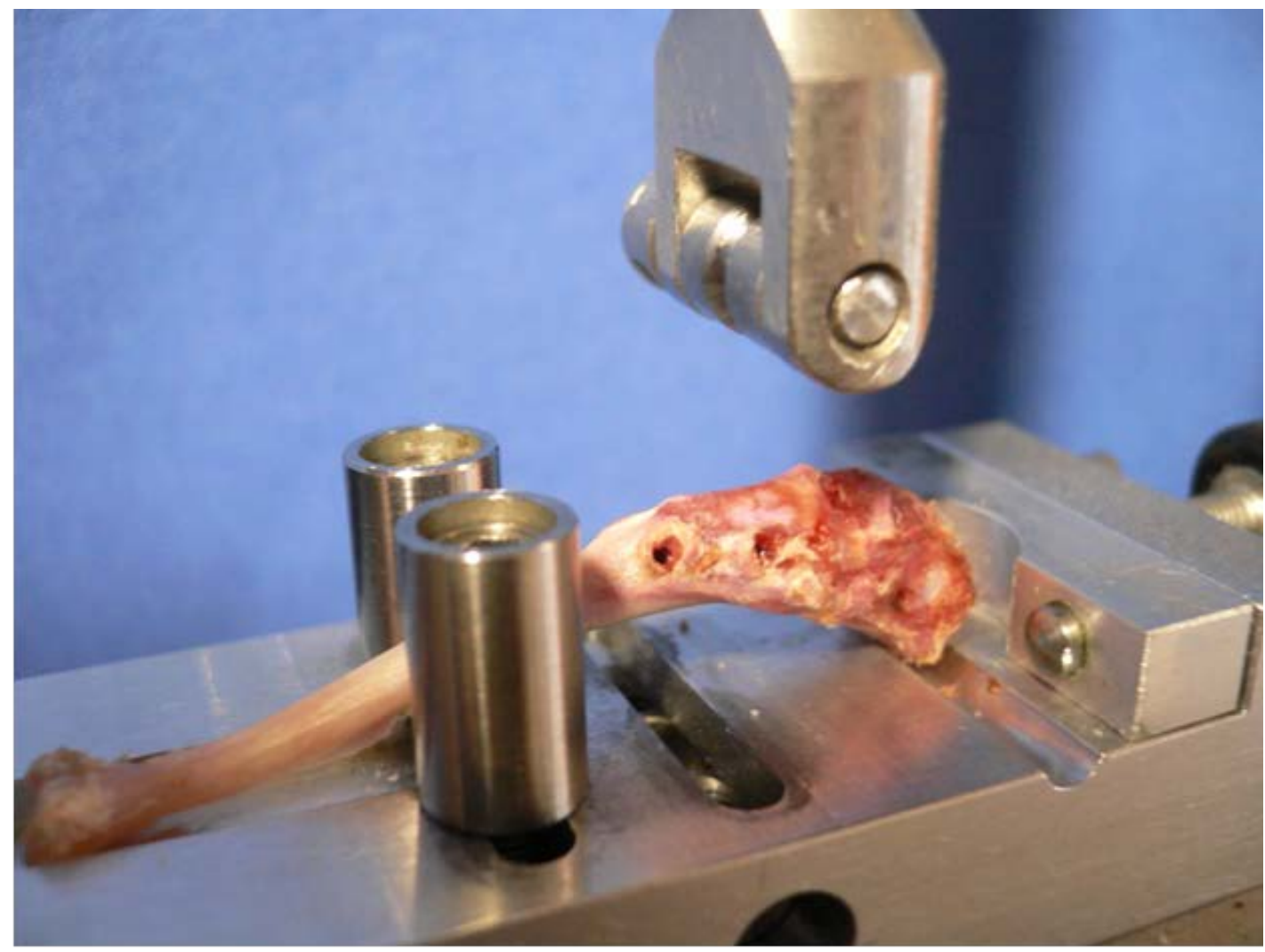

Abbildung 6: Versuchsaufbau des Biegetests mit Lagerungsblock, Rollstempel und Tibia. Zwei der vier Lagerungsbolzen wurden zur Verbesserung der Übersicht entfernt.

\subsubsection{Messparameter des biomechanischen Biegetests}

\subsubsection{Steifheit des Kallus}

Die in dem beschriebenen Biegetest ermittelten Kraft-Weg-Diagramme weisen einen charakteristischen, schon 2006 von Stuermer et al. beschriebenen Kurvenverlauf auf: Mit zunehmender Kraft kommt es zunächst zu einem linearen Anstieg der Kurve als Ausdruck einer elastischen Verformung des Knochens, die im Wesentlichen auf eine reversible Dehnung kollagener Fasern zurückzuführen ist. Wird die Belastung in dieser Phase beendet, so nimmt er seine ursprüngliche Form wieder an. Die Steigung der Kurve entspricht dabei der Steifheit bzw. Biegefestigkeit des Knochens: Je steiler sie verläuft, desto mehr Kraft muss aufgewendet werden, um eine definierte Verbiegung der Tibia zu bewirken. 


\subsubsection{Maximale Belastbarkeit des Kallus}

Mit weiterer Zunahme der Kraft kommt es zu Mikrofrakturen und Trabekelbrüchen und damit zu einer plastischen, irreversiblen Deformierung des Knochens. Die für eine definierte Verformung der Tibia erforderliche Kraft wird kleiner, der anfangs lineare Kurvenverlauf flacht sich ab. Wie Stuermer et al. 2006 zeigen konnten, ist von einer irreversiblen, plastischen Verformung des Knochens auszugehen, wenn der Kurvenverlauf den Bereich der doppelten Standardabweichung der Regressionsgeraden verlässt. Dieser Punkt im Kurvenverlauf wurde als Streckgrenze definiert (engl.: Yield Load), die damit der Kraft entspricht, die ein Knochen maximal ertragen kann ohne strukturelle, bleibende Schäden davonzutragen.

Wird die Kraft nach Überschreiten der Streckgrenze weiter gesteigert, so erreicht der Kurvenverlauf ein Maximum, das einem abrupten Abfall des Graphen als Ausdruck einer kompletten Fraktur des Knochens vorausgeht. Vor Erreichen dieser Maximalkraft wurde der Biegeversuch von der Software unseres Programms, wie schon oben erwähnt, automatisch abgebrochen, so dass eine Fraktur des Kallus in allen Fällen vermieden werden konnte.

\subsubsection{Validierung des biomechanischen Biegetests}

Vor Durchführung des biomechanischen Biegetests war dessen Reliabilität in einem Vorversuch validiert worden:

Im Rahmen dieses Vorversuchs wurde der beschriebene biomechanische Biegetest an jeweils beiden Tibiae einer Gruppe von 10 Ratten durchgeführt, die in Größe und Gewicht den Versuchstieren der Hauptuntersuchung glichen.

Nach Bestimmung von Steifheit und maximaler Belastbarkeit beider Tibiae wurden die intraindividuellen Abweichungen beider Parameter errechnet. Die Abweichungen lagen bei beiden Parametern im Mittel unter $15 \%$, was im Rahmen eines intraindividuellen Rechts-Links-Vergleichs als physiologisch anzusehen ist (Fisk und Baigent 1975; Budsberg et al. 1993). 


\section{$2.4 \quad$ Micro-CT}

\subsubsection{Scan der Tibiae}

Aus jeder der sechs Versuchsgruppen wurden randomisiert neun zufällig ausgewählte Tibiae einer Micro-CT-Untersuchung unterzogen.

Zu diesem Zweck wurden jeweils drei der insgesamt 54 Knochen, mit dem Tibiakopf nach unten, in einem in drei Kompartimente unterteilten zylindrischen Probenhalter positioniert und mit Schaumstoffkeilen fixiert (s. Abb. 7). In den Lagerungseinsatz eingefräste Markierungen erlaubten dabei eine Identifizierung der einzelnen Knochen.

Zur späteren Kalibrierung wurde in den Zylinder außerdem ein Dichtephantom gelegt, welches ein Knochen-, ein Luft- und ein Wasseräquivalent enthielt.

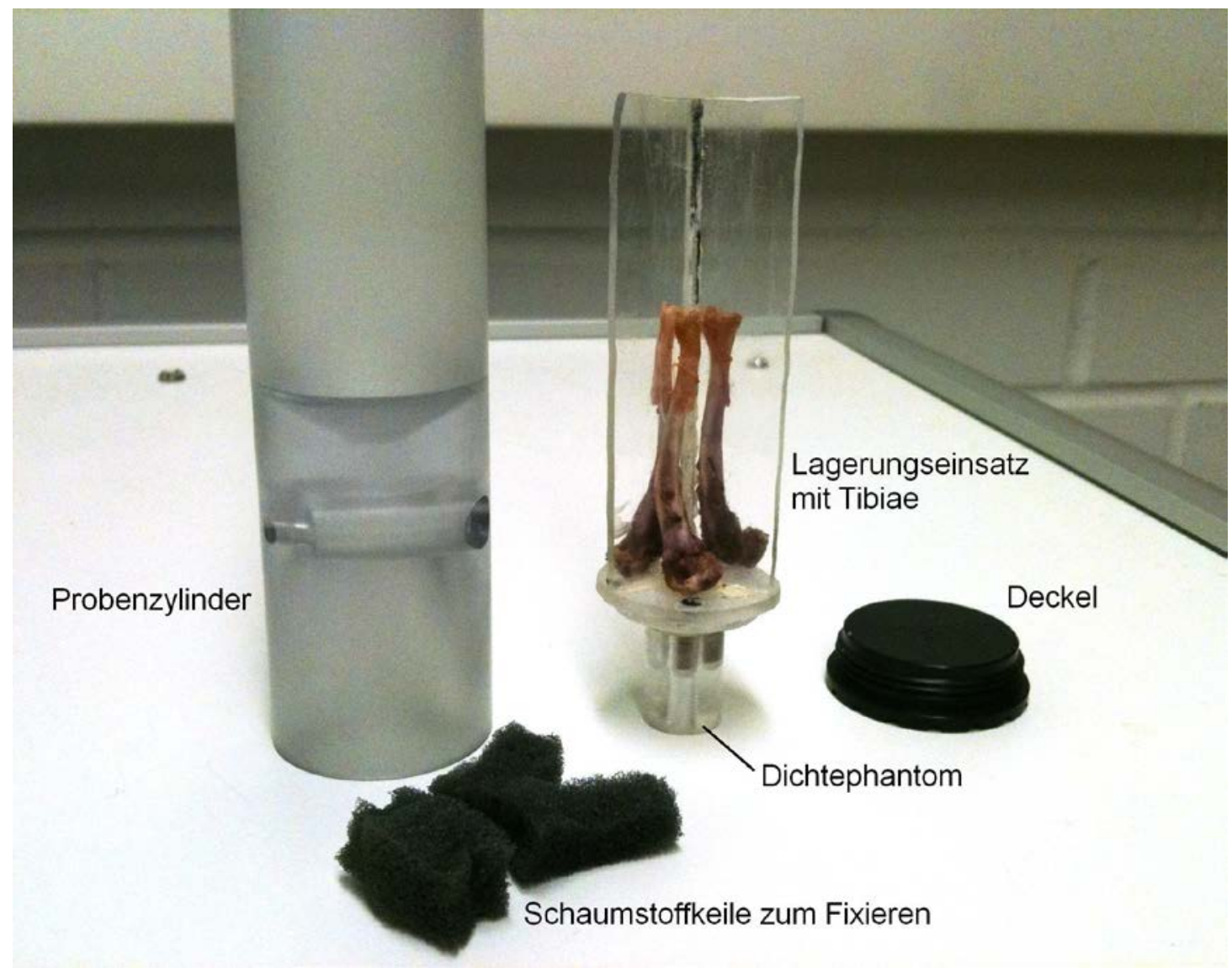

Abbildung 7: Geöffneter Probenhalter mit Tibiae und Dichtephantom 
Die Untersuchung erfolgte anschließend in einem eXplore Locus SP-Scanner (GE Healthcare, London, Ontario, Canada) (McNamara et al. 2005; Johnson et al. 2006). Das Scanprotocoll war mit 360 Grad Scan, 900 Projektionen pro Rotation, 72 kVp, 90 $\mathrm{mA}$, binning 2 und $1600 \mathrm{~ms}$ Belichtungszeit für alle Aufnahmen identisch.

Nach abgeschlossenem Scan wurden zur Planung der Detailrekonstruktion 3-D Bilder der einzelnen Tibiae mit dem „MicroView“-Programm v2.1.2 der Firma GE Healthcare erstellt.

Die 3-D Rekonstruktion erfolgte anschließend mit der dem System zugehörigen Software (evsbeam) unter Verwendung des Filtered Backprojektion Algorithm (FBP). Die rekonstruierte Auflösung der Datensätze betrug $0,03 \mathrm{~mm}$ isotrop.

\subsubsection{Morphometrische Auswertung der Micro-CT-Untersuchung}

Nach Rekonstruktion der Daten wurden diese mit dem 2010 im Rahmen eines DFGProjektes (STU 478/3-1) entwickelten „3DOsteoAnalyze“-Programm v1.00.3 zunächst hinsichtlich Knochendichte und Knochenvolumen ausgewertet, wobei die genannten Parameter in der von Ann-Lieber 1987 beschriebenen Weise berechnet wurden.

Das dreidimensionale Bild der Tibia wurde dazu im Raum jeweils so ausgerichtet, dass ein definierter Messrahmen exakt von dorsal an die Tibia angelegt werden konnte. Breite und Tiefe des Messrahmens wurden dabei mit jeweils $9 \mathrm{~mm}$ so gewählt, dass der gesamte Tibiaquerschnitt im Rahmen erfasst wurde. Die Höhe des Messfeldes wurde auf jeweils 2,5 mm proximal und distal des Osteotomiespaltes begrenzt. Durch Zentrierung des Rahmens auf den Frakturspalt wurde damit de facto eine $5 \mathrm{~mm}$ hohe Knochenscheibe analysiert, durch deren Mitte der Spalt verlief (s. Abb. 8). 


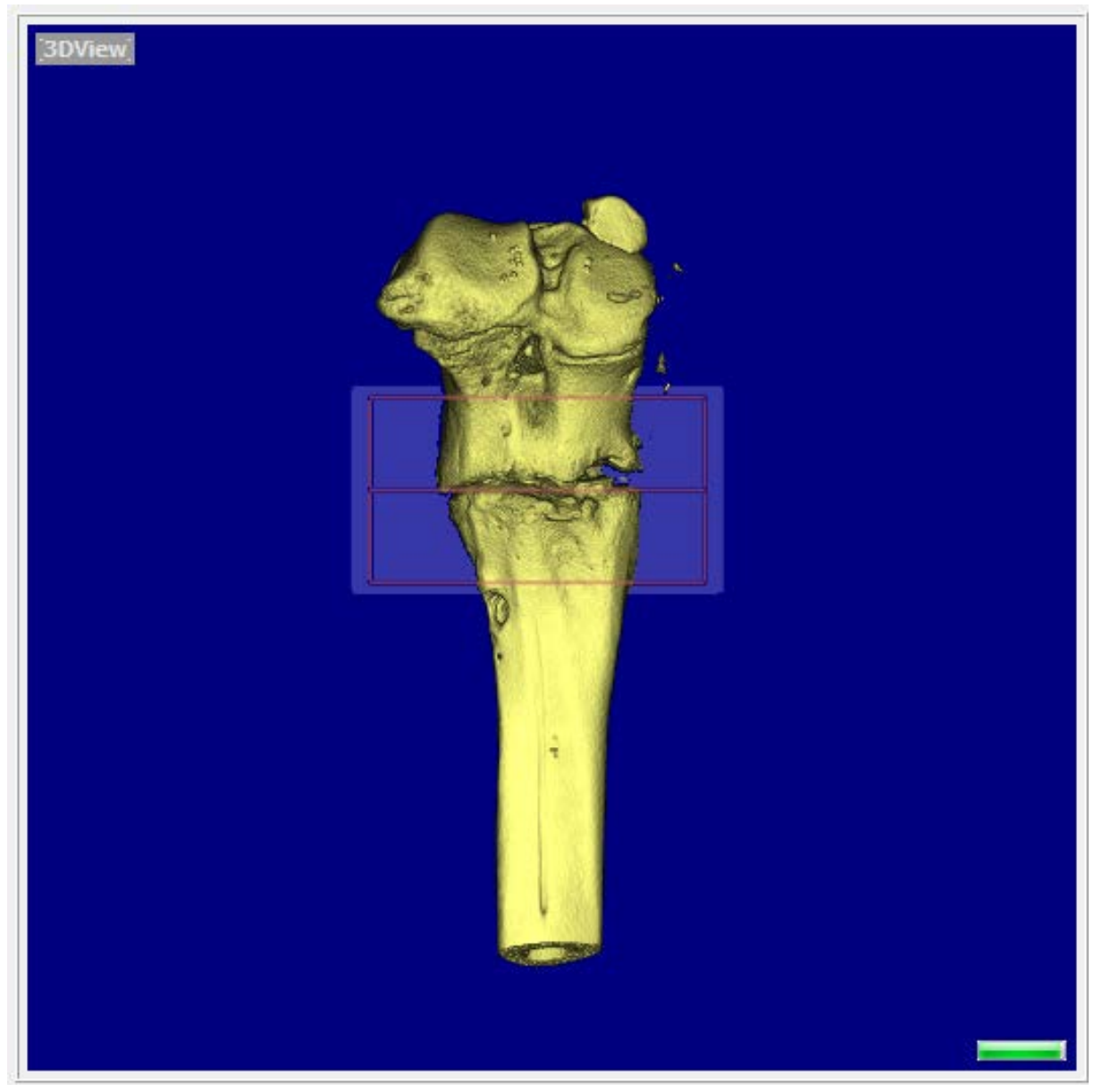

Abbildung 8: Rattentibia mit Messrahmen im 3DOsteoAnalyze-Programm

Mit dem Programm konnte anschließend von dieser Knochenscheibe ein Grauwerthistogramm erstellt werden, das auf der x-Achse die Helligkeitsstufen der Micro-CT und auf der y-Achse die Anzahl der zugehörigen Bildpunkte darstellte. Aus den unterschiedlichen Helligkeitsstufen konnte auf die Mineralisierung des Knochens geschlossen werden, weil diese mit der Abschwächung der Röntgenstrahlung in der CT korreliert. Der im Wesentlichen viergipflige Kurvenverlauf des Histogramms (s. Abb. 9a) entsprach dabei den sich überlappenden Grauwertbereichen von Luft, Weichteilgewebe, nichtkortikalem Knochen und kortikalem Knochen. Nach Subtraktion des ersten, der Luft entsprechenden Kurvengipfels verblieben die Grauwertbereiche von Weichteilgewebe, nichtkortikalem und kortikalem Knochen als jetzt dreigipfliges Histogramm (s. Abb. 9b). 


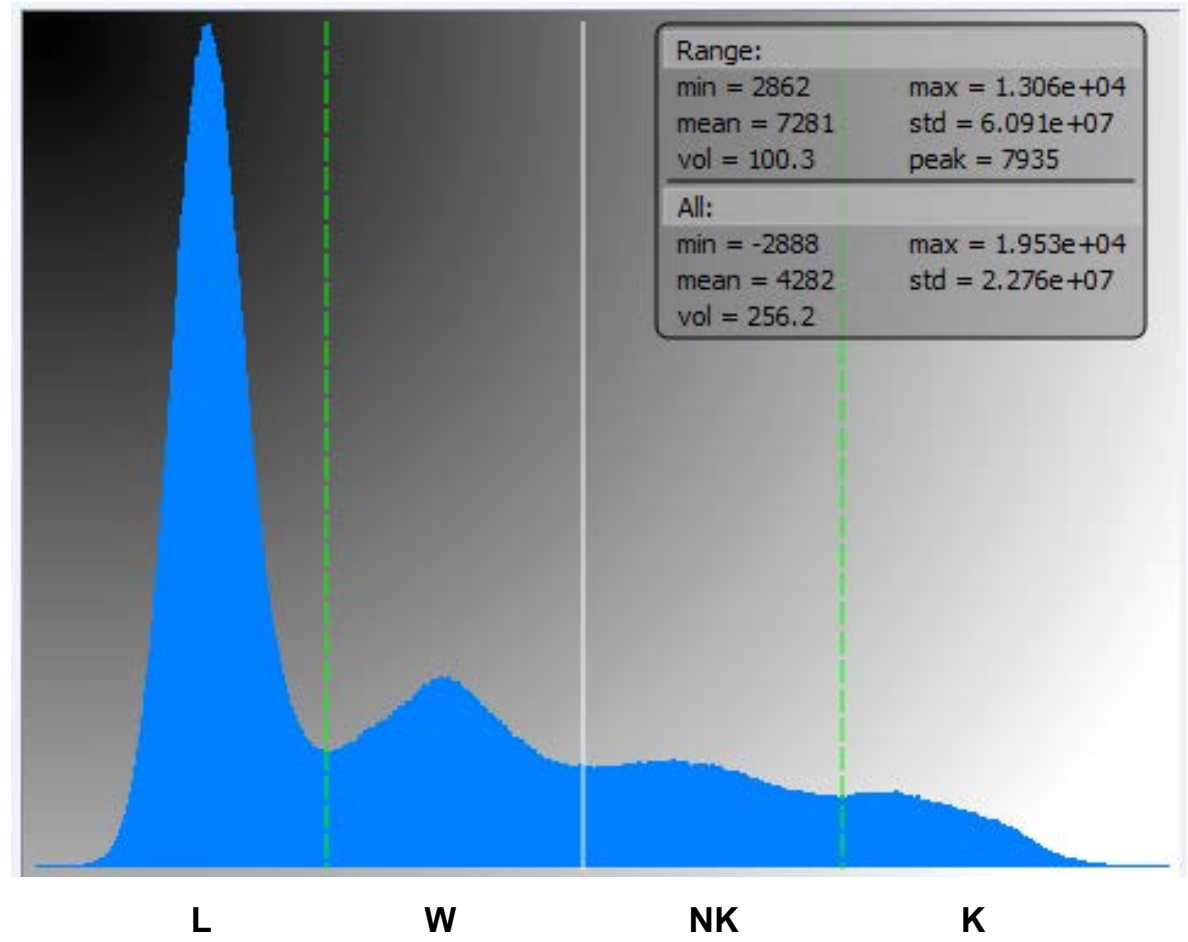

Abbildung 9a: Viergipfliges Histogramm mit den Grauwertbereichen von Luft (L), Weichteilgewebe (W), nichtkortikalem Knochen (NK) und kortikalem Knochen (K)

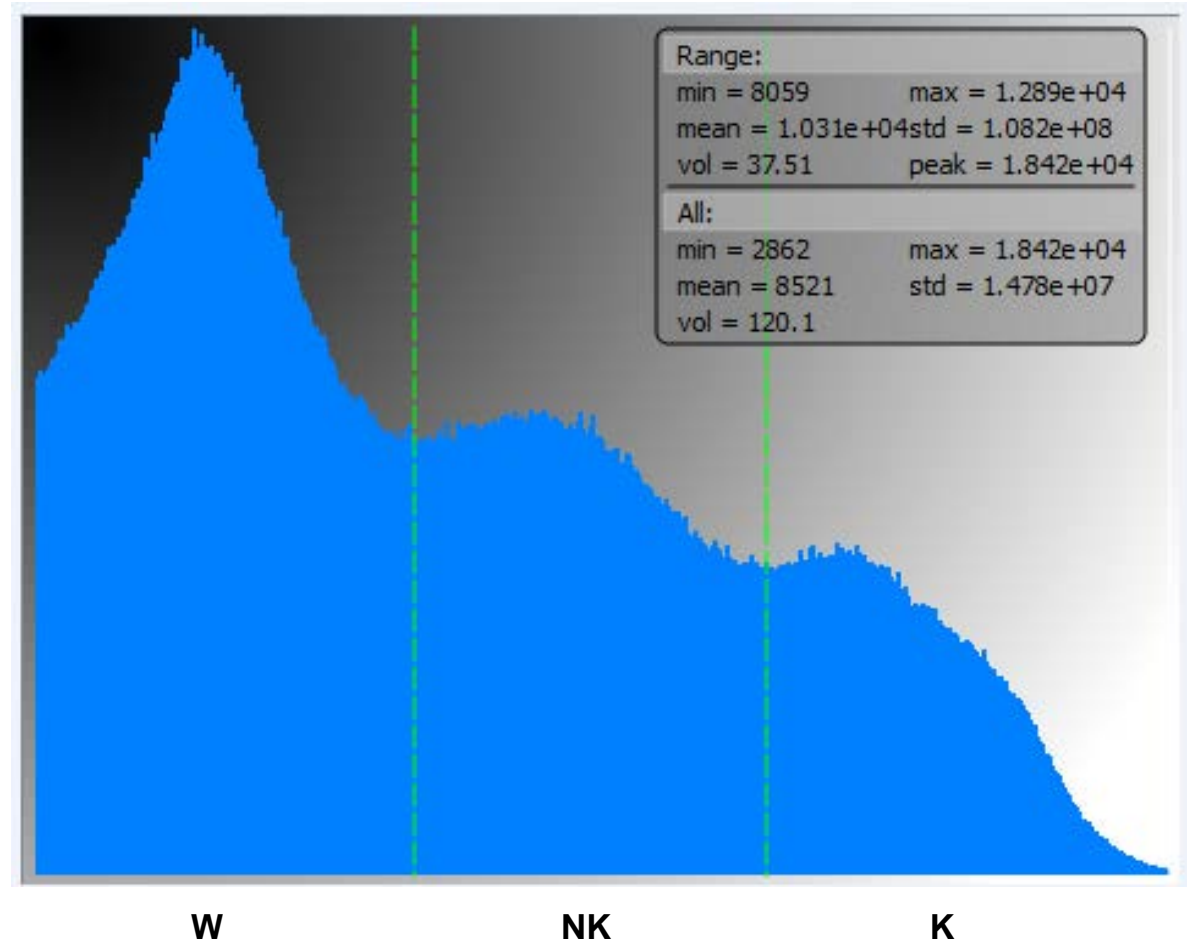

Abbildung 9b: Dreigipfliges Histogramm mit den Grauwertbereichen von Weichteilgewebe (W), nichtkortikalem (NK) und kortikalem Knochen (K) 
Der nichtkortikale Knochen ist dabei als Kallus und Trabekelgewebe anzusehen. Da wir in unmittelbarer Frakturnähe keine größeren Mengen trabekulären Gewebes erwarteten und im Micro-CT-Bild auch nicht visualisieren konnten, haben wir uns entschlossen, den nichtkortikalen Knochen vereinfachend als Kallusgewebe zu betrachten.

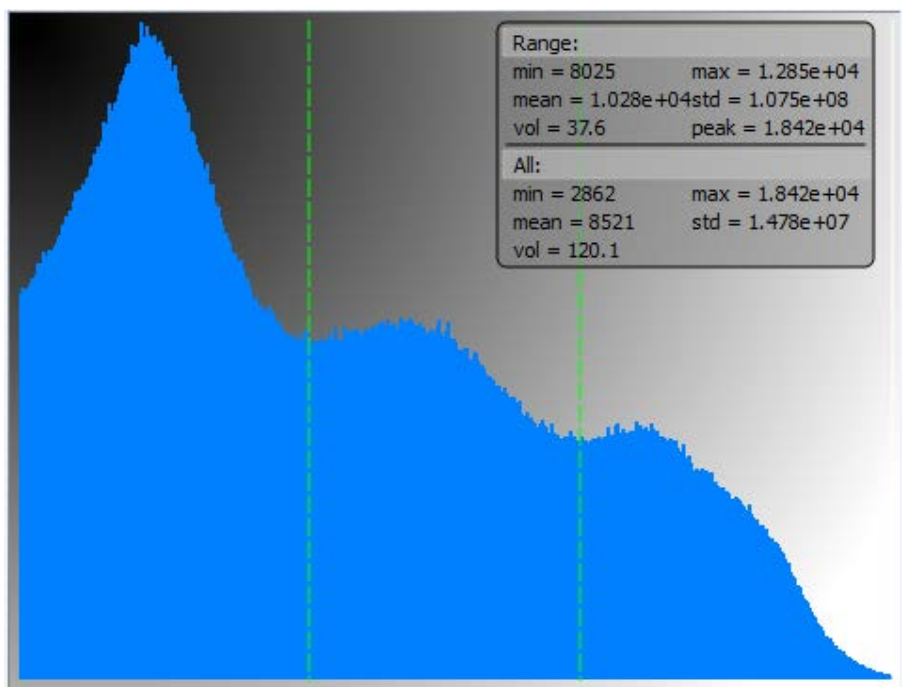

NK

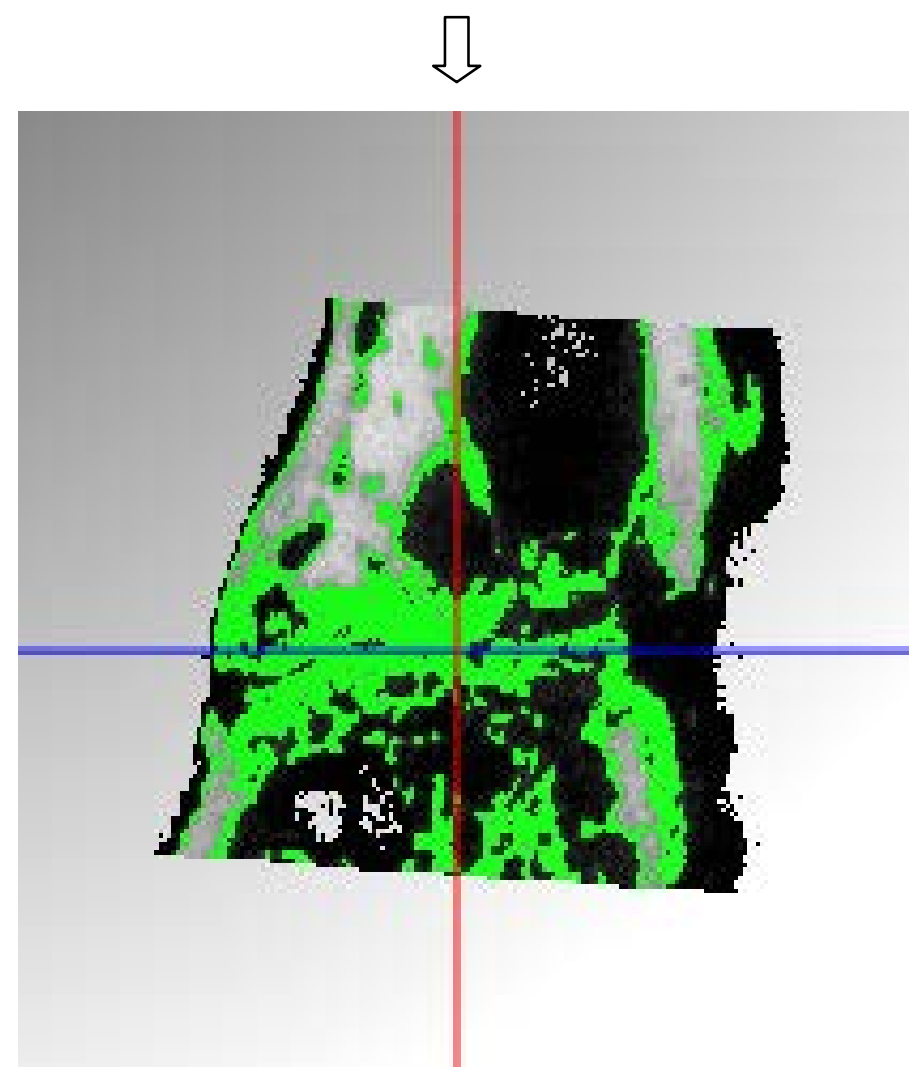

Abbildung 10: Der im Histogramm zwischen den Linien markierte Grauwertbereich des nichtkortikalen Knochens (NK) wird vom OsteoAnalyze-Programm im unteren Bild farbkodiert in grün dargestellt. Es wird deutlich, dass der nichtkortikale Knochen im Wesentlichen dem Kallusgewebe entspricht. 
Da die Abschwächung der Röntgenstrahlung zwar mit dem Grad der Mineralisierung des Knochens korreliert, nicht aber direkt mit dessen Massendichte, war zur Ermittlung der Knochendichte der Einsatz eines Dichtephantoms aus knochenäquivalentem Material definierter Dichten erforderlich (Valencia et al. 2006).

\subsubsection{Messparameter der Micro-CT-Untersuchung}

Von Kallusgewebe, Kortikalis und Gesamtknochen als Summe der beiden erstgenannten Bereiche wurden schließlich jeweils Knochendichte und Volumen bestimmt. Dazu wurde der Mittelwert des jeweils zugehörigen Histogrammbereichs mit Hilfe des Phantoms in Knochendichtewerte (BMD) umgerechnet und das Volumen als Produkt von Voxelanzahl und Voxelvolumen angegeben.

Zusätzlich wurde auch das Volumen des Gesamtgewebes (Gesamtknochen + Weichteilgewebe) ermittelt, das als Rechengröße für den nächsten Parameter diente: Der Knochenanteil am Gesamtgewebevolumen konnte damit anschließend als Quotient aus Gesamtknochenvolumen und Gesamtgewebevolumen berechnet und als „bone volume fraction“ (BV/TV) in Prozent angegeben werden. 
Tabelle 2: Messparameter der Micro-CT-Untersuchung

\begin{tabular}{|c|c|}
\hline Messparameter [Einheit] & Berechnungsgrundlage \\
\hline Gesamtknochendichte $\left[\mathrm{g} / \mathrm{cm}^{3}\right]$ & $\begin{array}{l}\text { arithmetisches Mittel aus Kortikalisdichte } \\
\text { und Kallusdichte }\end{array}$ \\
\hline Kortikalisdichte $\left[\mathrm{g} / \mathrm{cm}^{3}\right]$ & $\begin{array}{l}\text { Knochendichte des kortikalen } \\
\text { Knochenanteils }\end{array}$ \\
\hline Kallusdichte $\left[\mathrm{g} / \mathrm{cm}^{3}\right]$ & $\begin{array}{l}\text { Knochendichte des nichtkortikalen } \\
\text { Knochenanteils }\end{array}$ \\
\hline Gesamtknochenvolumen [mm $\left.{ }^{3}\right]$ & $\begin{array}{l}\text { Summe von Kortikalisvolumen und } \\
\text { Kallusvolumen }\end{array}$ \\
\hline Kortikalisvolumen $\left[\mathrm{mm}^{3}\right]$ & Volumen des kortikalen Knochenanteils \\
\hline Kallusvolumen [mm $\left.{ }^{3}\right]$ & $\begin{array}{l}\text { Volumen des nichtkortikalen } \\
\text { Knochenanteils }\end{array}$ \\
\hline Gesamtgewebevolumen [mm $\left.{ }^{3}\right]$ & $\begin{array}{l}\text { Summe von Gesamtknochenvolumen } \\
\text { und Weichteilgewebevolumen }\end{array}$ \\
\hline bone volume fraction (BV/TV) [\%] & $\begin{array}{l}\text { Quotient aus Gesamtknochenvolumen } \\
\text { und Gesamtgewebevolumen }\end{array}$ \\
\hline
\end{tabular}




\section{$2.5 \quad$ Mikroradiographie}

\subsubsection{Histologische Aufarbeitung und Anfertigung der Mikroradiographien}

Nach Abschluss der Micro-CT-Untersuchung wurden dieselben Tibiae in einer aufsteigenden Alkoholreihe entwässert und entfettet, indem sie zweimal 3 Tage in 40\%-, zweimal 5 Tage in 70\%-, 5 Tage in 80\%- und 2 Tage in 96\%igem Ethanol gelagert wurden. Die folgenden 2 Tage wurden die Tibiae einem 1:1 Gemisch aus 96\%igem Ethanol und Methylmethacrylat (MMA) ausgesetzt, 2 weitere Tage schließlich reinem Methylmethacrylat. Abschließend wurden die Tibiae einzeln in ein Gemisch aus $1000 \mathrm{ml}$ Methylmethacrylat, $200 \mathrm{ml}$ Dibutylphthalat und $29 \mathrm{~g}$ Benzoylperoxid eingebettet, wobei für jede Tibia eine $80 \times 30 \mathrm{~mm}$ große Rollrandflasche mit einem Volumen von $40 \mathrm{ml}$ verwendet wurde. Nach dem mehrere Wochen in Anspruch nehmenden Aushärten der MMA-Blöcke wurden diese aus den Flaschen gelöst und mit einer Innenlochsäge (Leica SP 1600 Sägemikrotom, Leica Instruments $\mathrm{GmbH}$, Nussloch, Deutschland) in 8 bis 12 jeweils $150 \mu \mathrm{m}$ dicke Schnitte zertrennt. Die Schnitte wurden dabei als Längsschnitte der Tibia im rechten Winkel zur Plattenebene ausgeführt und verliefen damit parallel zur unter 2.1.4 definierten Schraubenebene.

Aus den so erstellten sagittalen Schnitten jeder Tibia wurden jeweils 3 zentrale, aufeinander folgende histologische Präparate ausgewählt und mit einem FaxitronRöntgengerät (Model-Nr. 43855A, Faxitron X-ray system, Hewlett Packard, San Diego, USA) auf Kodak Professional Film geröntgt (Kodak Industrex SR 45, ISO 9002, Rochester, New York, USA). Bei einer Röhrenspannung von 10 kV und einer Stromstärke von 0,3 mA betrug die Belichtungszeit dabei 3 Minuten (Stuermer et al. 2010 b). Die Filme wurden nach abgeschlossener Fixierung getrocknet, beschriftet und archiviert.

Für die spätere Auswertung der polychromen Sequenzmarkierung (siehe 2.6, S. 43) wurden die radiographierten Präparate dann mit Eukitt ${ }^{\circledR}$ (Kindler, Freiburg, Deutschland) auf $76 \times 52 \mathrm{~mm}$ großen Objektträgern fixiert und archiviert. 


\subsubsection{Digitalisierung der Mikroradiographien}

Die Mikroradiographien wurden anschließend mit einem Leica Stereomakroskop (Leica MZ 7-5, Bensheim, Deutschland) analysiert, wobei eine Kaltlichtlampe als Lichtquelle diente (Leica KL 1500 LCD, Bensheim, Deutschland). Mithilfe einer Kamera (Leica DC 300F, Bensheim, Deutschland) konnten die Bilder dann in den Computer (Intel Pentium 4, 2.6 GHz) eingelesen werden. In Vorversuchen waren die dafür möglichen Einstellungen von Makroskop, Kamera und Software ausgetestet worden, wobei sich die folgende Konfiguration als ideal erwiesen hatte:

Am Makroskop wurde stets ein 1,0er Objektiv verwendet, welches bei guter Ausnutzung der Monitorgröße eine vollständige Abbildung der auszuwertenden Strukturen gestattete.

Die Temperatur der Halogenlampe betrug konstant $3000 \mathrm{~K}$.

Für die mechanische Blende der Kaltlichtlampe wurde die Schalterposition B gewählt, wodurch eine mäßige Helligkeit erreicht wurde (A: minimale Blendenweite, E: maximale Blendenweite).

Die Belichtungszeit wurde für jedes Bild individuell angepasst, um so die unterschiedliche Helligkeit der Mikroradiographien auszugleichen, die auf minimale Unterschiede in der Schnittdicke der histologischen Präparate zurückzuführen war. Dabei wurde der Bereich zwischen 345 und 460 ms nicht verlassen.

Alle Bilder wurden bei der Digitalisierung so positioniert, dass die proximale Tibia am Monitor oben, die distale Tibia unten, die ventromediale Tibiaseite links und die dorsale Tibiaseite rechts abgebildet wurde (s. Abb. 11). Ein standardisierter, $5 \mathrm{~mm}$ hoher Messrahmen mit einer genau mittig verlaufenden, horizontalen Ziellinie wurde so über dem Bild ausgerichtet, dass die horizontale Ziellinie exakt auf der Kortikalis des distalen Tibiasegmentes zu liegen kam.

Die digitalisierten Bilder konnten anschließend mit der Software Leica Quantimet QWin 2003 (Leica, Bensheim, Deutschland) bearbeitet und nach einem standardisierten, eigens für diese Aufgabe programmierten Algorithmus histomorphometrisch ausgewertet werden. 


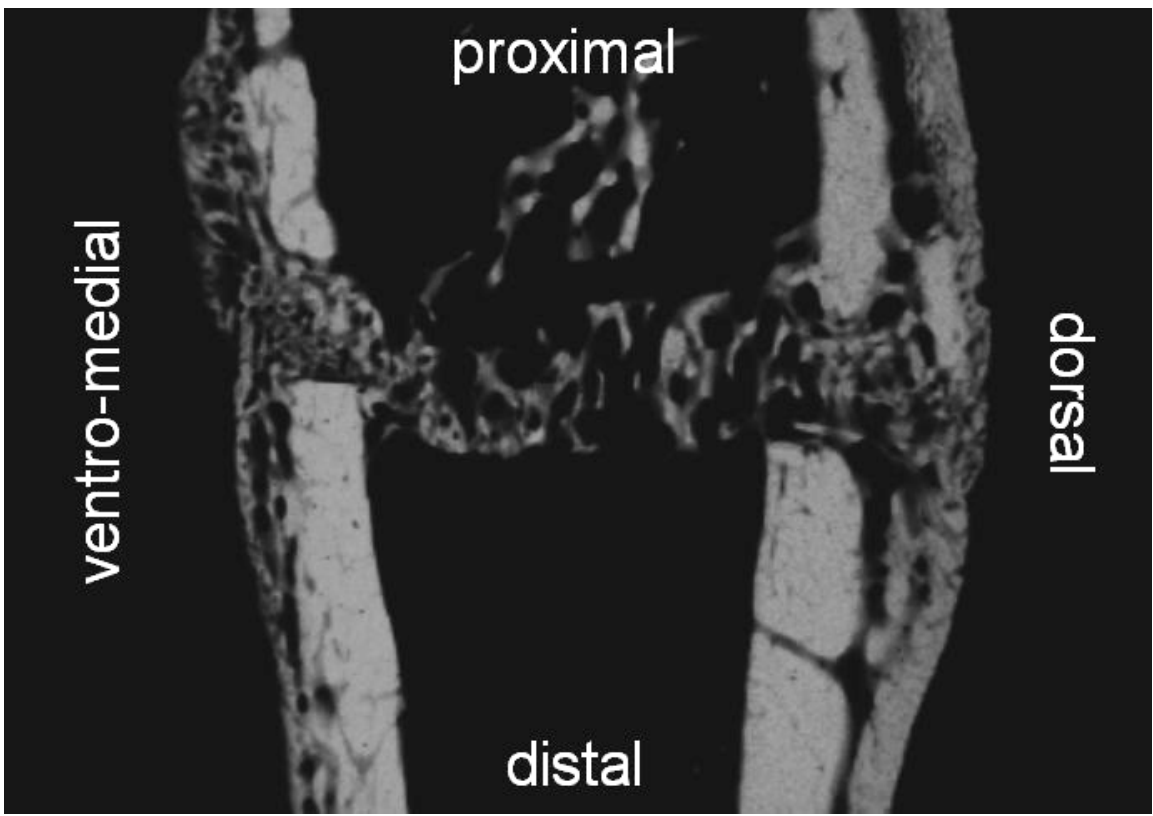

Abbildung 11: Digitalisierte Mikroradiographie mit Kennzeichnung der räumlichen Ausrichtung

\subsubsection{Morphometrische Auswertung der Mikroradiographien}

\subsubsection{Graudetektion}

Die histomorphometrische Auswertung erfolgte in zufälliger Reihenfolge verblindet, so dass dem Untersucher die Gruppenzugehörigkeit der jeweils analysierten Mikroradiographie nicht bekannt war.

Vor Identifizierung aller zu vermessenden Knochenanteile wurde zunächst eine automatische Graudetektion durchgeführt, bei der die verwendete Software alle von ihr als Knochen erkannten Bereiche blau anfärbte. Die Detektionsempfindlichkeit wurde anschließend durch den Untersucher manuell so lange korrigiert, bis alle knöchernen Strukturen blau dargestellt waren und damit von der Software als Knochen erfasst wurden. Eine Fehlinterpretation durch Über- oder Unterdetektion des Programms konnte so vermieden werden. 

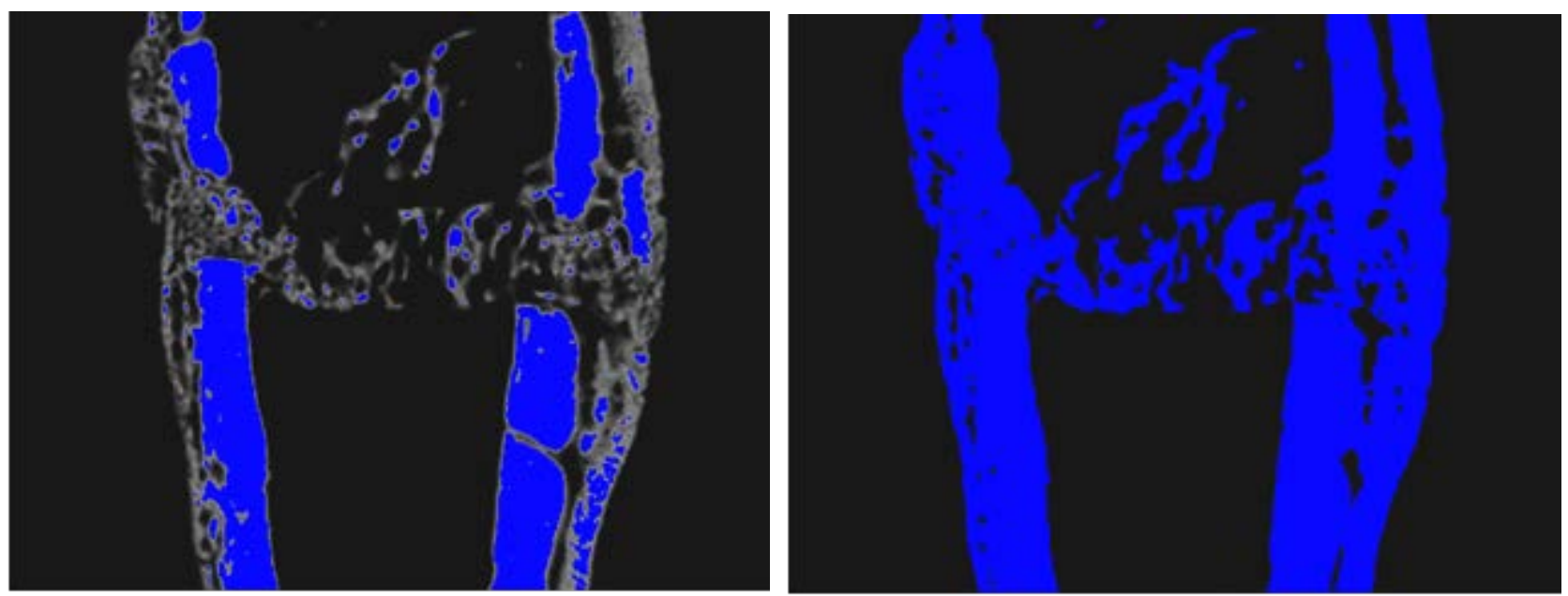

Abbildung 12: Graudetektion vor und nach manueller Korrektur. Nach automatischer Graudetektion erscheinen die als Knochen erkannten Bereiche blau, nicht detektierter Knochen wird grau dargestellt (linkes Bild). Nach manueller Korrektur sind alle knöchernen Stukturen blau markiert (rechtes Bild).

\subsubsection{Flächendefinition}

Im nächsten Arbeitsschritt wurden die im mikroradiographischen Bild differenzierbaren Knochenanteile definiert, indem sie mit dem Cursor manuell umfahren wurden. Die gewählte Flächenbegrenzung wurde dabei als Linie dargestellt, so dass bedarfsweise eine spätere Korrektur des Linienverlaufs stets vorgenommen werden konnte. Eine Mehrfachzuordnung desselben Areals zu verschiedenen Knochenflächen wurde dabei durch das Programm verhindert.

Auf diese Weise wurde zunächst die gesamte Knochenfläche einschließlich des Kallus über den Messrahmen hinaus mit dem Cursor umfahren und damit detektiert, wobei abgesprengte und dislozierte Knochenanteile von der Messung ausgeschlossen wurden.

Anschließend wurde die Kortikalis, die durch ihre hohe Dichte leicht vom restlichen Knochen abgegrenzt werden kann, mit dem Cursor umrandet. Dabei wurden proximale ventromediale Kortikalisfläche, proximale dorsale Kortikalisfläche, distale 
ventromediale Kortikalisfläche und distale dorsale Kortikalisfläche jeweils getrennt voneinander umfahren und damit definiert.

Im nächsten Schritt wurde die Kallusfläche definiert, wobei ventromediale, dorsale und endostale Kallusfläche wieder einzeln erfasst wurden (s. Abb. 13). Die Abgrenzung des periostalen Kallus war dabei schon in den vorausgehenden Arbeitsschritten bei Festlegung der Knochengesamtfläche und der Kortikalisfläche erfolgt. Auch der endostale Kallus war bereits durch die innere Kortikalisbegrenzung partiell eingegrenzt. Durch seine höhere Knochendichte und kompaktere Struktur war er leicht von der proximal und distal an ihn angrenzenden spongiösen Trabekelfläche abzugrenzen. Die Trennung zwischen periostalem und endostalem Kallus erfolgte durch eine mittige Verbindungslinie zwischen proximaler und distaler Kortikalis.

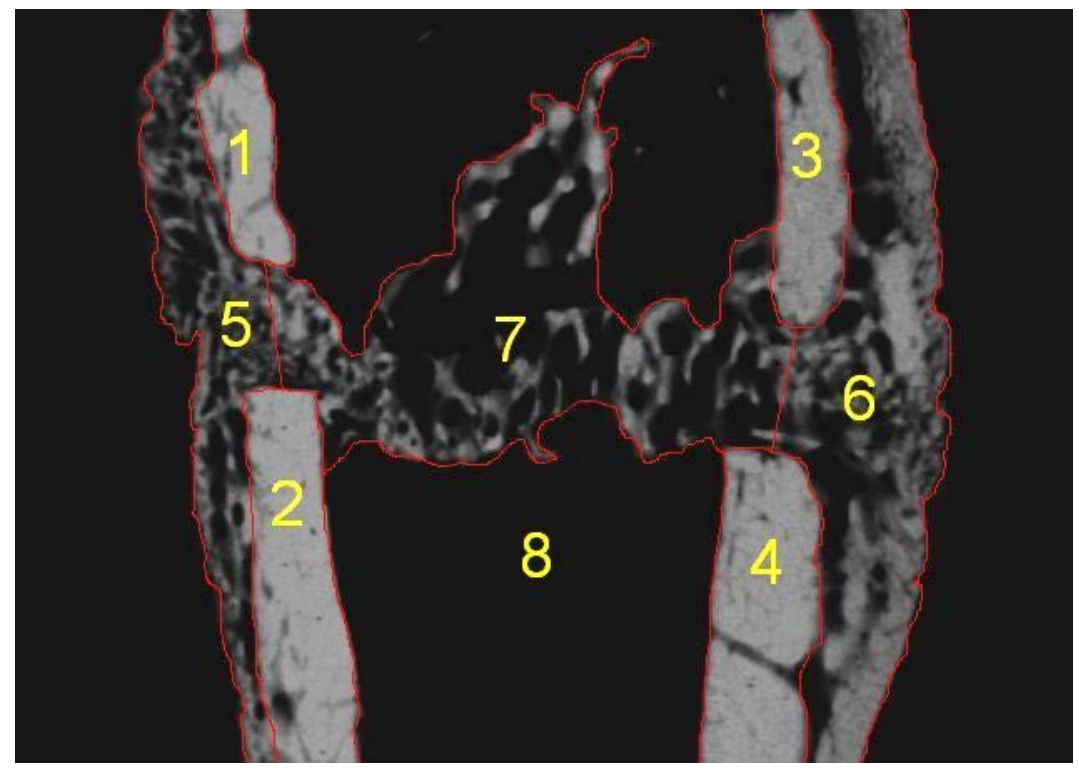

Abbildung 13: Flächendefinition im mikroradiographischen Bild. 1=Kortikalisfläche proximal ventromedial, $2=$ Kortikalisfläche distal ventromedial, $3=$ Kortikalisfläche proximal dorsal, 4=Kortikalisfläche distal dorsal, 5=Kallusfläche ventromedial, 6=Kallusfläche dorsal, 7= Kallusfläche endostal, 8=Trabekelfläche distal

Die Definition der Trabekelfläche ergab sich schließlich aus den schon festgelegten Abgrenzungen zur Kortikalis und zum endostalen Kallus hin. Soweit sich innerhalb der Trabekelfläche abgesprengte oder dislozierte Knochenanteile befanden, wurden diese von der Detektion ausgeschlossen. 


\subsubsection{Vektorgestützte Knochendickemessung}

Nach Differenzierung der einzelnen Knochenanteile wurde die Dicke von Kortikalis und Kallus in den verschiedenen Bildregionen ermittelt. Zu diesem Zweck wurden vom Untersucher zunächst jeweils 5 Vektoren manuell so über die osteotomienahe Kortikalis gelegt, dass sie senkrecht zur Knochenlängsachse ausgerichtet waren und die Kortikalis beidseits überragten (s. Abb. 14 links). Die Länge der Vektoren wurde dann von der eingesetzten Software auf die zuvor definierte Kortikalisfläche begrenzt, wobei die computergesteuerte Einkürzung der Vektoren vom Untersucher visuell überprüft- und bei Bedarf korrigiert wurde. Auf diese Weise wurde die Dicke der distalen ventromedialen- und der distalen dorsalen Kortikalis exakt bestimmt.

Anschließend wurden 10 Vektoren orthogonal zur Knochenlängsachse durch den ventromedialen periostalen Kallus beidseits der Osteotomie gelegt, deren Länge dann von der Software auf die zuvor ermittelte Kallusdicke eingekürzt wurde (s. Abb. 14 rechts). Nach Messung der ventromedialen Kallusdicke wurde die dorsale Kallusdicke in gleicher Weise ermittelt.
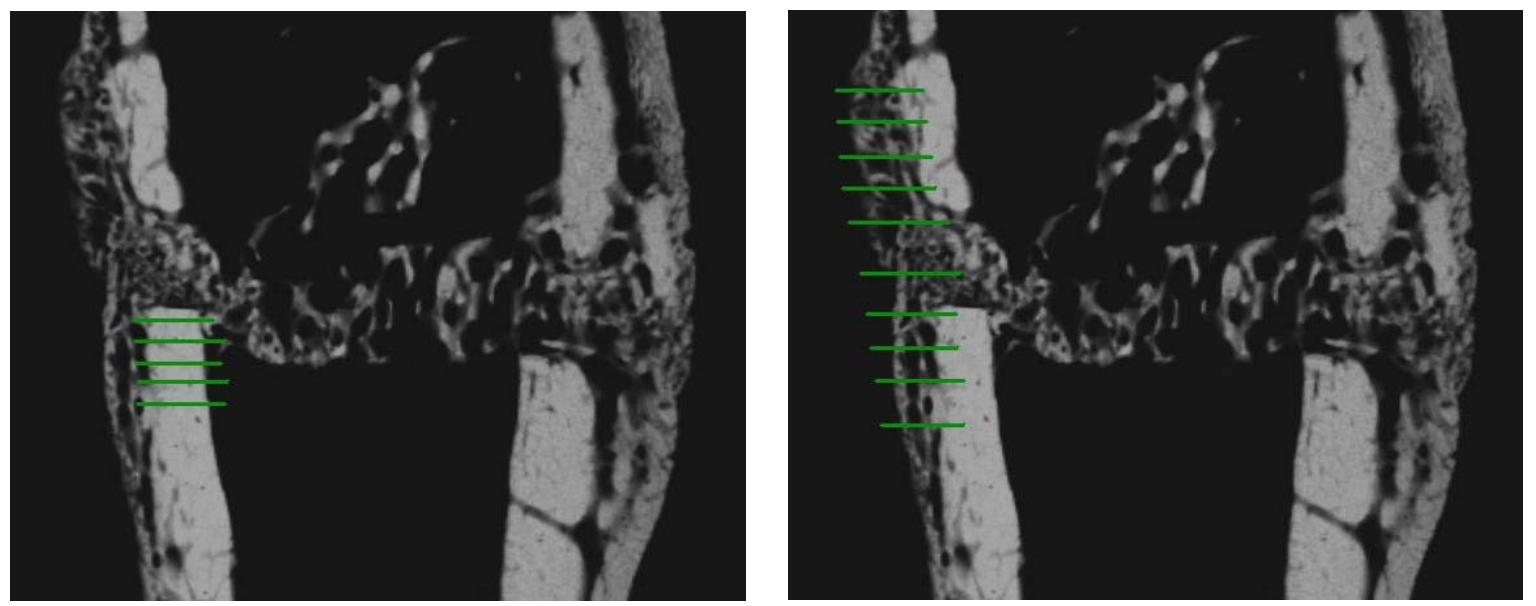

Abbildung 14: Vektorgestützte Knochendickemessung. Kortikalisdicke distal ventromedial (linkes Bild), Kallusdicke ventromedial (rechtes Bild) 


\subsubsection{Validierung der Auswertung}

Die morphometrische Auswertung der Mikroradiographien umfasst zahlreiche Arbeitsschritte, die vom Untersucher manuell durchgeführt oder visuell überprüft werden und wird dadurch in hohem Maße durch den Untersucher beeinflusst. Um untersucherbedingte Messschwankungen soweit wie möglich zu reduzieren, wurden alle Mikroradiographien durch denselben Untersucher ausgewertet.

Vor der Untersuchung wurde die Reproduzierbarkeit der Messergebnisse zudem evaluiert, indem die Auswertung von fünf Mikroradiographien an fünf aufeinander folgenden Tagen jeweils wiederholt wurde. Die Messergebnisse durften dabei um nicht mehr als $\pm 10 \%$ vom jeweiligen Mittelwert abweichen, was für alle Messparameter erreicht werden konnte.

\subsubsection{Messparameter der Mikroradiographie}

Aus den im Rahmen der morphometrischen Auswertung gewonnenen Daten berechnete die Software unseres Programms für jeden einzelnen Tibiaschnitt die durchschnittliche Dicke von Kortikalis und Kallus. Außerdem wurde die Dichte von Kortikalis, Kallus und Trabekelfläche ermittelt, indem für jede der entsprechend definierten Flächen der prozentuale Knochenanteil berechnet wurde. Schließlich bestimmte die Software die Anzahl der Trabekelkreuzungen absolut und pro $\mathrm{mm}^{2}$ sowie die mittlere Trabekeldicke. Von den die Trabekelflächen und die Kortikalis betreffenden Parametern, die proximal und distal der Frakturebene erfasst werden konnten, wurden zur Vereinfachung jeweils nur die distal gemessenen Werte ausgewertet. Die einzelnen Messparameter und ihre Berechnung sind in Tabelle 3 aufgeführt. 
Tabelle 3: Mikroradiographisch ermittelte Messparameter und ihre Berechnung

\begin{tabular}{|c|c|}
\hline Messparameter [Einheit] & Berechnungsgrundlage \\
\hline Kortikalisdicke ventromedial [mm] & $\begin{array}{l}\text { arithmetisches Mittel der } 5 \text { durch die distale } \\
\text { ventromediale Kortikalis gelegten Vektoren }\end{array}$ \\
\hline Kortikalisdicke dorsal [mm] & $\begin{array}{l}\text { arithmetisches Mittel der } 5 \text { durch die distale } \\
\text { dorsale Kortikalis gelegten Vektoren }\end{array}$ \\
\hline Kallusdicke ventromedial [mm] & $\begin{array}{l}\text { arithmetisches Mittel der } 5 \text { durch den ventro- } \\
\text { medialen Kallus gelegten Vektoren }\end{array}$ \\
\hline Kallusdicke dorsal [mm] & $\begin{array}{l}\text { arithmetisches Mittel der } 5 \text { durch den dorsalen } \\
\text { Kallus gelegten Vektoren }\end{array}$ \\
\hline Kortikalisdichte ventromedial [\%] & $\begin{array}{l}\text { prozentualer Knochenanteil der als "Kortikalis } \\
\text { distal ventromedial" definierten Fläche }\end{array}$ \\
\hline Kortikalisdichte dorsal [\%] & $\begin{array}{l}\text { prozentualer Knochenanteil der als "Kortikalis } \\
\text { distal dorsal" definierten Fläche }\end{array}$ \\
\hline Kallusdichte ventromedial [\%] & $\begin{array}{l}\text { prozentualer Knochenanteil der als "Kallus } \\
\text { ventromedial" definierten Fläche }\end{array}$ \\
\hline Kallusdichte dorsal [\%] & $\begin{array}{l}\text { prozentualer Knochenanteil der als "Kallus } \\
\text { dorsal" definierten Fläche }\end{array}$ \\
\hline Kallusdichte endostal [\%] & $\begin{array}{l}\text { prozentualer Knochenanteil der als "Kallus } \\
\text { endostal“ definierten Fläche }\end{array}$ \\
\hline Trabekelflächendichte [\%] & $\begin{array}{l}\text { prozentualer Knochenanteil der als "Trabekel- } \\
\text { fläche distal" definierten Fläche }\end{array}$ \\
\hline Anzahl Trabekelkreuzungen absolut & $\begin{array}{l}\text { absolute Anzahl der Trabekelkreuzungen im } \\
\text { Bereich der distalen Trabekelfläche }\end{array}$ \\
\hline Anzahl Trabekelkreuzungen pro $\mathrm{mm}^{2}\left[1 / \mathrm{mm}^{2}\right]$ & $\begin{array}{l}\text { Anzahl der Trabekelkreuzungen pro } \mathrm{mm}^{2} \mathrm{im} \\
\text { Bereich der distalen Trabekelfläche }\end{array}$ \\
\hline Mittlere Trabekeldicke $[\mu \mathrm{m}]$ & $\begin{array}{l}\text { arithmetisches Mittel der Trabekeldurchmesser } \\
\text { im Bereich der distalen Trabekelfläche }\end{array}$ \\
\hline
\end{tabular}




\subsection{Polychrome Sequenzmarkierung}

\subsubsection{Prinzip der polychromen Sequenzmarkierung}

Während die unter 2.2, 2.4 und 2.5 beschriebenen radiologischen Verfahren lediglich statische Momentaufnahmen des Knochenpräparates liefern, ermöglicht die polychrome Sequenzmarkierung eine Darstellung der Dynamik des Knochenumbaus während der Frakturheilung. Mit ihrer Hilfe lassen sich Informationen über den zeitlichen Ablauf der Knochenneubildung unter der Vibrationsbehandlung gewinnen.

Zu diesem Zweck waren den Tieren zu definierten Zeitpunkten der Frakturheilung verschiedenfarbige Fluorochrome wie unter 2.1.1 beschrieben injiziert worden. Diese Farbstoffe verbinden sich mit Kalzium zu fluoreszierenden Chelatkomplexen, die in den aktuell mineralisierten Knochen eingelagert werden, im vorliegenden Fall vor allem in den Frakturkallus. Durch die zeitlich versetzte Applikation der verschiedenfarbigen Fluoreszenzfarbstoffe bilden sich im Kallus farblich entsprechend markierte Banden aus. Nach der unter 2.5.1 beschriebenen Aufarbeitung und Fixierung der Tibiae können diese Banden dann im Rahmen einer quantitativen fluoreszenzmikroskopischen Analyse identifiziert und einer definierten Zeitspanne der Frakturheilung zugeordnet werden (Benneteau-Pelissero et al. 2000).

\subsubsection{Fluorochrome}

In Anlehnung an Rahn (1976) wurden für die polychrome Sequenzmarkierung die Fluorochrome Xylenolorange-Tetranatriumsalz (XO), Calcein-Grün (CG), Alizarinkomplexon (AK) und Tetracyclinhydrochlorid (TC) eingesetzt (alle: Merck, Darmstadt, Deutschland).

Die Fluorochrome wurden in destilliertem Wasser (XO, CG, AK) bzw. isotoner Kochsalzlösung (TC) gelöst und den Tieren dann zu definierten Zeitpunkten subkutan injiziert. Die jeweiligen Dosierungen und Applikationstage der verschiedenen Farbstoffe sind Tabelle 4 zu entnehmen. Tabelle 4 gibt auch Auskunft 
über die zu erwartende Farbe der einzelnen Fluorochrome und über die Zeitspanne der Frakturheilung, die durch jede der Farben repräsentiert wird. Diese Zeitspanne entspricht jeweils dem Zeitraum zwischen zwei aufeinander folgenden Fluorochromapplikationen, da der Farbstoff immer nur in den Knochenanteil eingelagert werden kann, der sich zum Zeitpunkt der Injektion neu gebildet hat und der noch nicht durch einen anderen Fluoreszenzfarbstoff besetzt ist.

Tabelle 4: Dosierung, Applikationszeitpunkt, markierte Zeitspanne und Farbe der einzelnen Fluorochrome

\begin{tabular}{|c|c|c|c|c|}
\hline \hline $\begin{array}{c}\text { Fluoro- } \\
\text { chrom }\end{array}$ & $\begin{array}{c}\text { Dosierung } \\
(\mathrm{mg} / \mathrm{kg} \mathrm{KG})\end{array}$ & $\begin{array}{c}\text { Applikationstag } \\
\text { nach Osteotomie }\end{array}$ & $\begin{array}{c}\text { Markierte Zeitspanne } \\
\text { (Tag nach Osteotomie })\end{array}$ & $\begin{array}{c}\text { Fluoreszenz- } \\
\text { farbe }\end{array}$ \\
\hline \hline XO & 90 & 13 & $0-13$ & Orange \\
\hline CG & 10 & 18 & $14-18$ & Grün \\
\hline AK & 30 & 24,26 & $19-26$ & Rot \\
\hline TC & 25 & 35 & $27-35$ & Gelb \\
\hline
\end{tabular}

$\mathrm{XO}=$ Xylenolorange-Tetranatriumsalz, CG = Calcein-Grün, AK= Alizarinkomplexon, TC $=$ Tetracyclinhydrochlorid

\subsubsection{Digitalisierung der polychromen Sequenzmarkierung}

Die wie unter 2.5.1 beschrieben präparierten und fixierten Knochenschnitte wurden mit einem Auflicht-Fluoreszenz-Stereomakroskop (Leica Stereomakroskop, MZ 7-5 mit FluoCombi III, Bensheim, Deutschland) unter Blaulicht betrachtet und ausgewertet, wobei eine Quecksilberhöchstdrucklampe mit einer Leistung von 50 Watt (Leica KL 1500 LCD, Bensheim, Deutschland) als Lichtquelle diente. Um die erforderliche Fluorochromanregung durch das Blaulicht zu gewährleisten, wurde zusätzlich ein Anregungsfilter für den Wellenlängenbereich von 450-490 nm eingesetzt. Mithilfe der schon zuvor verwendeten Kamera (Leica DC 300F, Bensheim, Deutschland) konnten die Bilder dann wieder in den Computer (Intel 
Pentium 4, 2.6 GHz) eingelesen werden. In Vorversuchen hatte sich die folgende Konfiguration von Makroskop, Kamera und Software als ideal erwiesen:

Am Makroskop wurde wieder ein 1,0er Objektiv verwendet, das eine vollständige Darstellung der Präparate bei guter Ausnutzung der Monitorgröße ermöglicht.

Die Belichtungszeit wurde wieder individuell angepasst, um so die unterschiedliche Helligkeit der auf dem Monitor dargestellten Bilder auszugleichen, die auf minimale Unterschiede in der Schnittdicke der Präparate zurückzuführen war. Dabei wurden stets Zeiten zwischen 9,7 und 12,9 s gewählt.

Mit Hilfe eines in der Software enthaltenen Gain-Reglers wurde die Bildhelligkeit um den Faktor 3 verstärkt.

Alle Präparate wurden bei der Digitalisierung wieder so positioniert, dass die proximale Tibia am Monitor oben, die distale Tibia unten, die ventromediale Tibiaseite links und die dorsale Tibiaseite rechts abgebildet wurde. Ein standardisierter, $5 \mathrm{~mm}$ hoher Messrahmen mit einer genau mittig verlaufenden, horizontalen Ziellinie wurde so über dem Bild ausgerichtet, dass die horizontale Ziellinie exakt auf der Kortikalis des distalen Tibiasegmentes zu liegen kam.

Die digitalisierten Bilder konnten anschließend mit der Software Leica Quantimet QWin 2003 (Leica, Bensheim, Deutschland) bearbeitet und nach einem standardisierten, eigens für diese Aufgabe programmierten Algorithmus morphometrisch ausgewertet werden.

\subsubsection{Morphometrische Auswertung der polychromen Sequenzmarkierung}

\subsubsection{Flächendefinition}

Die morphometrische Auswertung erfolgte wieder in zufälliger Reihenfolge verblindet, so dass dem Untersucher die Gruppenzugehörigkeit des jeweils analysierten Bildes nicht bekannt war.

Dabei wurden auch im fluoreszenzmikroskopischen Bild die differenzierbaren Knochenanteile wie unter 2.5.3.2 beschrieben definiert: 
Zunächst wurde wieder die gesamte Knochenfläche einschließlich des Kallus über den Messrahmen hinaus mit dem Cursor umfahren und damit detektiert, wobei abgesprengte Knochenstücke von der Detektion ausgeschlossen blieben.

Anschließend wurden proximale ventromediale Kortikalisfläche, proximale dorsale Kortikalisfläche, distale ventromediale Kortikalisfläche und distale dorsale Kortikalisfläche jeweils getrennt voneinander umfahren und damit definiert. Da in der Kortikalis kaum Umbauvorgänge stattfinden, zeigt sie im Gegensatz zum Kallus eine nur geringe Fluoreszenz.

Im nächsten Schritt wurde die für die polychrome Sequenzmarkierung letztlich relevante Kallusfläche definiert, wobei ventromediale, dorsale und endostale Kallusfläche wieder einzeln erfasst wurden. Die Abgrenzung des periostalen Kallus ergab sich dabei wieder schon aus den vorausgehenden Arbeitsschritten, auch der endostale Kallus war bereits durch die innere Kortikalisbegrenzung partiell eingegrenzt, durch seine höhere Knochendichte und vermehrte Fluoreszenz war er leicht von der proximal und distal an inn angrenzenden spongiösen Trabekelfläche abzugrenzen. Die Abgrenzung zwischen periostalem und endostalem Kallus erfolgte wieder durch eine mittig zwischen proximaler und distaler Kortikalis gezogene Grenzlinie.

\subsubsection{Flächen-Fluorochrom-Zuordnung}

Nach Definition des ventromedialen, des dorsalen und des endostalen Kallus wurden in diesem Gewebe mit Hilfe der unter 2.6.3 beschriebenen Apparatur die Areale mit identischen Fluoreszenzeigenschaften markiert und dem jeweiligen Fluorochrom zugeordnet. Die Zuordnung erfolgte dabei stets in der gleichen, von der Software vorgegebenen Reihenfolge ( $\mathrm{XO} \rightarrow \mathrm{CG} \rightarrow \mathrm{AK} \rightarrow \mathrm{TC}$ ). Soweit sich nicht fluoreszierende Bereiche innerhalb des Kallus fanden, wurden diese nicht berücksichtigt.

Bei der Auswertung der Präparate fanden sich nur kleine XO-markierte Areale, die zumeist von stark fluoreszierendem, CG-markiertem Knochen eingeschlossen waren. Diese XO-Areale konnten zwar bei hoher mikroskopischer Auflösung sichtbar gemacht werden, für eine Detektion und quantitative Analyse durch die eingesetzte 
Software war ihre Fluoreszenz aber zu schwach. Die quantitative, fluoreszenzmikroskopische Analyse konnte deshalb nur für CG-, AK- und TC-Areale durchgeführt werden.

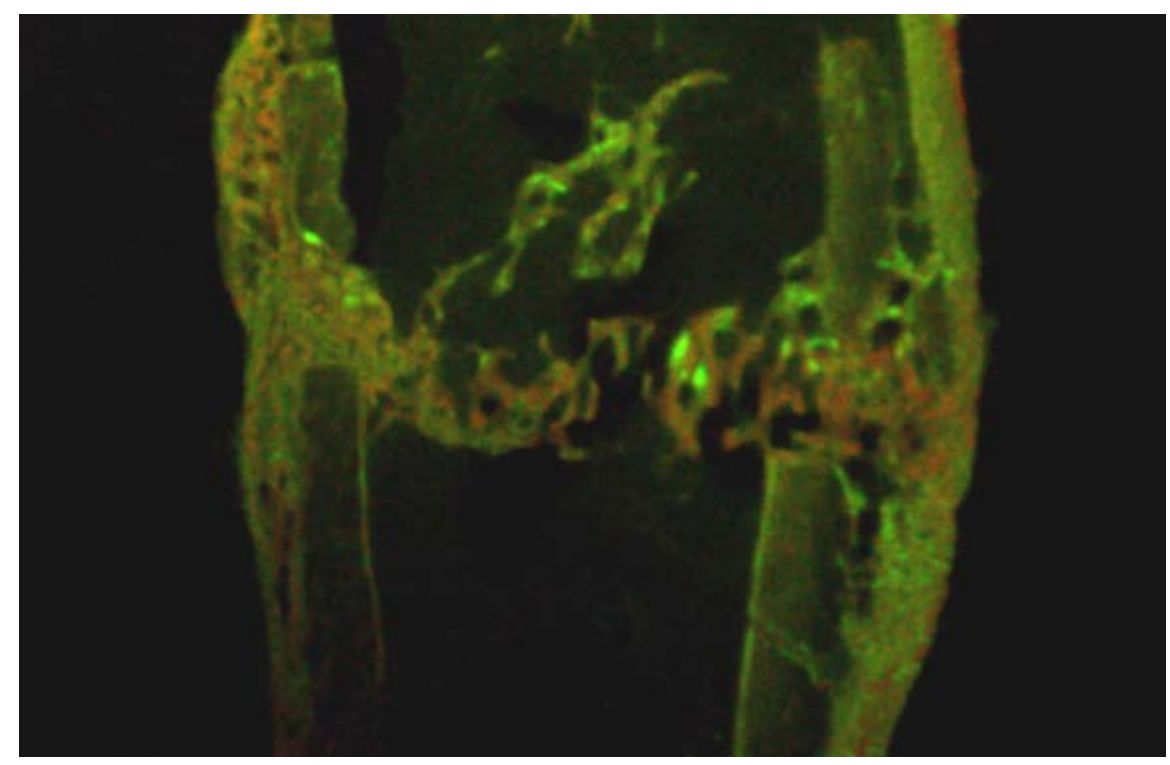

Abbildung 15: Fluoreszenzmikroskopisches Bild. CG-markierter Knochen wird grün-, AK-markierter Knochen rot- und TC-markierter Knochen gelb dargestellt.

\subsubsection{Validierung der Auswertung}

Die morphometrische Auswertung der polychromen Sequenzmarkierung beinhaltet zahlreiche Arbeitsschritte, die vom Untersucher manuell durchgeführt und damit durch ihn beeinflusst werden. Um untersucherbedingte Messschwankungen so weit wie möglich zu vermeiden, wurden alle Auswertungen durch denselben Untersucher durchgeführt.

Wie schon unter 2.5.3.4 beschrieben wurde die Reproduzierbarkeit der Messergebnisse zudem evaluiert, indem die Auswertung von fünf Präparaten an fünf aufeinander folgenden Tagen jeweils wiederholt wurde. Die Messergebnisse durften dabei um nicht mehr als $\pm 10 \%$ vom jeweiligen Mittelwert abweichen, was für alle Messparameter erreicht werden konnte. 


\subsubsection{Messparameter der polychromen Sequenzmarkierung}

Aus den im Rahmen der morphometrischen Auswertung gewonnenen Daten berechnete die Software unseres Programms für jeden einzelnen Tibiaschnitt die Gesamtkallusfläche und die verschiedenen fluorochrommarkierten Kallusflächen, jeweils in den Lokalisationen ventromedial, dorsal und endostal. Die einzelnen Messparameter und ihre Berechnung sind in Tabelle 5 aufgeführt.

Tabelle 5: Messparameter der polychromen Sequenzmarkierung

\begin{tabular}{|l|l|}
\hline \hline Messparameter [Einheit] & Berechnungsgrundlage \\
\hline \hline Gesamtfläche Kallus ventromedial $\left[\mathrm{mm}^{2}\right]$ & $\begin{array}{l}\text { absolute Fläche des gesamten } \\
\text { ventromedialen Kallus }\end{array}$ \\
\hline CG-Fläche Kallus ventromedial $\left[\mathrm{mm}^{2}\right]$ & $\begin{array}{l}\text { absolute Fläche des CG-markierten } \\
\text { ventromedialen Kallus }\end{array}$ \\
\hline AK-Fläche Kallus ventromedial $\left[\mathrm{mm}^{2}\right]$ & $\begin{array}{l}\text { absolute Fläche des AK-markierten } \\
\text { ventromedialen Kallus }\end{array}$ \\
\hline TC-Fläche Kallus ventromedial $\left[\mathrm{mm}^{2}\right]$ & $\begin{array}{l}\text { absolute Fläche des TC-markierten } \\
\text { ventromedialen Kallus }\end{array}$ \\
\hline Gesamtfläche Kallus dorsal $\left[\mathrm{mm}^{2}\right]$ & $\begin{array}{l}\text { absolute Fläche des gesamten } \\
\text { dorsalen Kallus }\end{array}$ \\
\hline CG-Fläche Kallus dorsal $\left[\mathrm{mm}^{2}\right]$ & $\begin{array}{l}\text { absolute Fläche des CG-markierten } \\
\text { dorsalen Kallus }\end{array}$ \\
\hline AK-Fläche Kallus dorsal $\left[\mathrm{mm}^{2}\right]$ & $\begin{array}{l}\text { absolute Fläche des AK-markierten } \\
\text { dorsalen Kallus }\end{array}$ \\
\hline TC-Fläche Kallus dorsal [mm²] & $\begin{array}{l}\text { absolute Fläche des TC-markierten } \\
\text { dorsalen Kallus }\end{array}$ \\
\hline Gesamtfläche Kallus endostal $\left[\mathrm{mm}^{2}\right]$ & $\begin{array}{l}\text { absolute Fläche des gesamten } \\
\text { endostalen Kallus }\end{array}$ \\
\hline CG-Fläche Kallus endostal $\left[\mathrm{mm}^{2}\right]$ & $\begin{array}{l}\text { absolute Fläche des CG-markierten } \\
\text { endostalen Kallus }\end{array}$ \\
\hline AK-Fläche Kallus endostal $\left[\mathrm{mm}^{2}\right]$ & $\begin{array}{l}\text { absolute Fläche des AK-markierten } \\
\text { endostalen Kallus }\end{array}$ \\
\hline TC-Fläche Kallus endostal $\left[\mathrm{mm}^{2}\right]$ & $\begin{array}{l}\text { absolute Fläche des TC-markierten } \\
\text { endostalen Kallus }\end{array}$ \\
\hline Additivkallus [mm²] & $\begin{array}{l}\text { Summe der gesamten ventromedialen, } \\
\text { dorsalen und endostalen Kallusflächen }\end{array}$ \\
\hline
\end{tabular}




\subsection{Statistik}

Alle Messergebnisse wurden einer statistischen Auswertung mit Hilfe des GraphPad Prism-Programms unterzogen (Version 4.00c, April 2003, GraphPad Software Inc., San Diego, USA).

Nach Testgruppen getrennt wurden dabei alle für die Messparameter des biomechanischen Biegetests (2.3.3), der Micro-CT-Untersuchung (2.4.3), der mikroradiographischen Analyse (2.5.4) und der polychromen Sequenzmarkierung (2.6.5) ermittelten Ergebnisse zunächst auf Gauß’sche Normalverteilung geprüft, die für alle Daten nachgewiesen werden konnte.

Zusätzlich wurden für alle Ergebnisse Mittelwert und Standardabweichung berechnet. Um die Unterschiede zwischen den ermittelten Daten der einzelnen Testgruppen auf Signifikanz zu überprüfen, wurden zunächst eine univariate Varianzanalyse (one-way-ANOVA) und anschließend ein Tukey-Kramer post-hocTest durchgeführt. Für alle Ergebnisse wurde ein Signifikanzniveau von $p<0,05$ als signifikant gewertet. 


\section{Ergebnisse}

\subsection{Futteraufnahme, Körpergewicht und Uterusgewicht der Tiere}

Nachfolgend sind zunächst die durchschnittliche tägliche Futteraufnahme und das Gewicht der Tiere während des Versuchsablaufs graphisch dargestellt, eine numerische Auflistung der einzelnen Werte findet sich zusätzlich in den Tabellen 7 und 8 im tabellarischen Anhang.

Zur Ermittlung der Werte war am Anfang jeder Versuchswoche zunächst das Gewicht der Ratten gemessen und am Ende der Woche der tägliche Futterverbrauch berechnet worden. Die Osteotomie der Rattentibiae war jeweils nach der achten Woche erfolgt.

\subsubsection{Futteraufnahme}

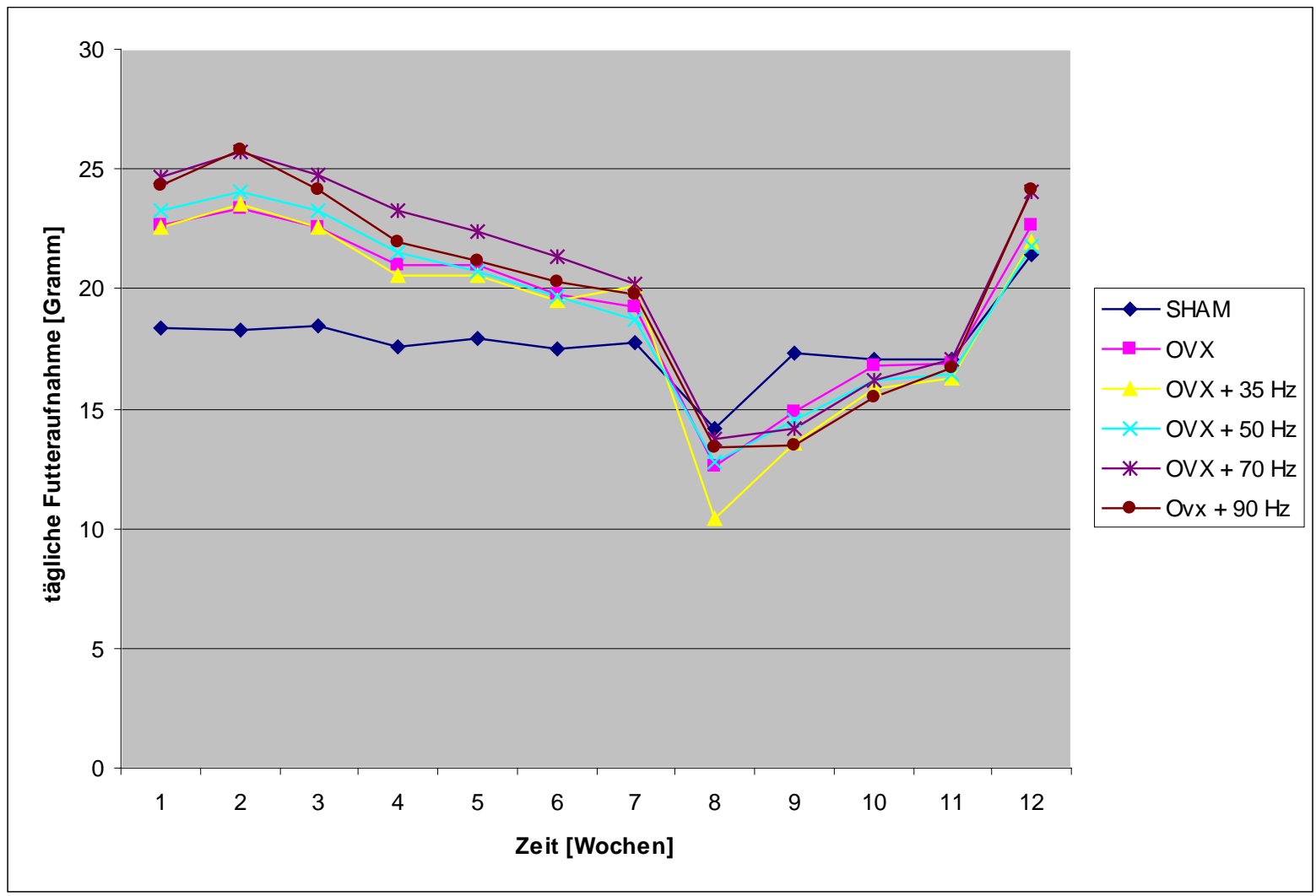

Abbildung 16: Durchschnittliche tägliche Futteraufnahme der Tiere im Versuchsverlauf 
Deutlich sichtbar ist die signifikant höhere Futteraufnahme der ovarektomierten Ratten im Vergleich zu den scheinoperierten, gesunden Tieren vor der Osteotomie. Nach dem Eingriff sinkt die Futteraufnahme der Ratten in allen Versuchsgruppen in ähnlicher Weise, um dann im Verlauf der nächsten 4 Wochen wieder anzusteigen, unabhängig davon, ob eine Vibrationsbehandlung durchgeführt wird oder nicht. Der schnellste Anstieg der Futteraufnahme auf ähnliche Werte wie vor der Osteotomie findet sich bei den SHAM-Tieren.

Die Futteraufnahme der vibrierten Ratten und die der nichtvibrierten Tiere der OVXGruppe unterscheiden sich nicht erkennbar.

\subsubsection{Körpergewicht}

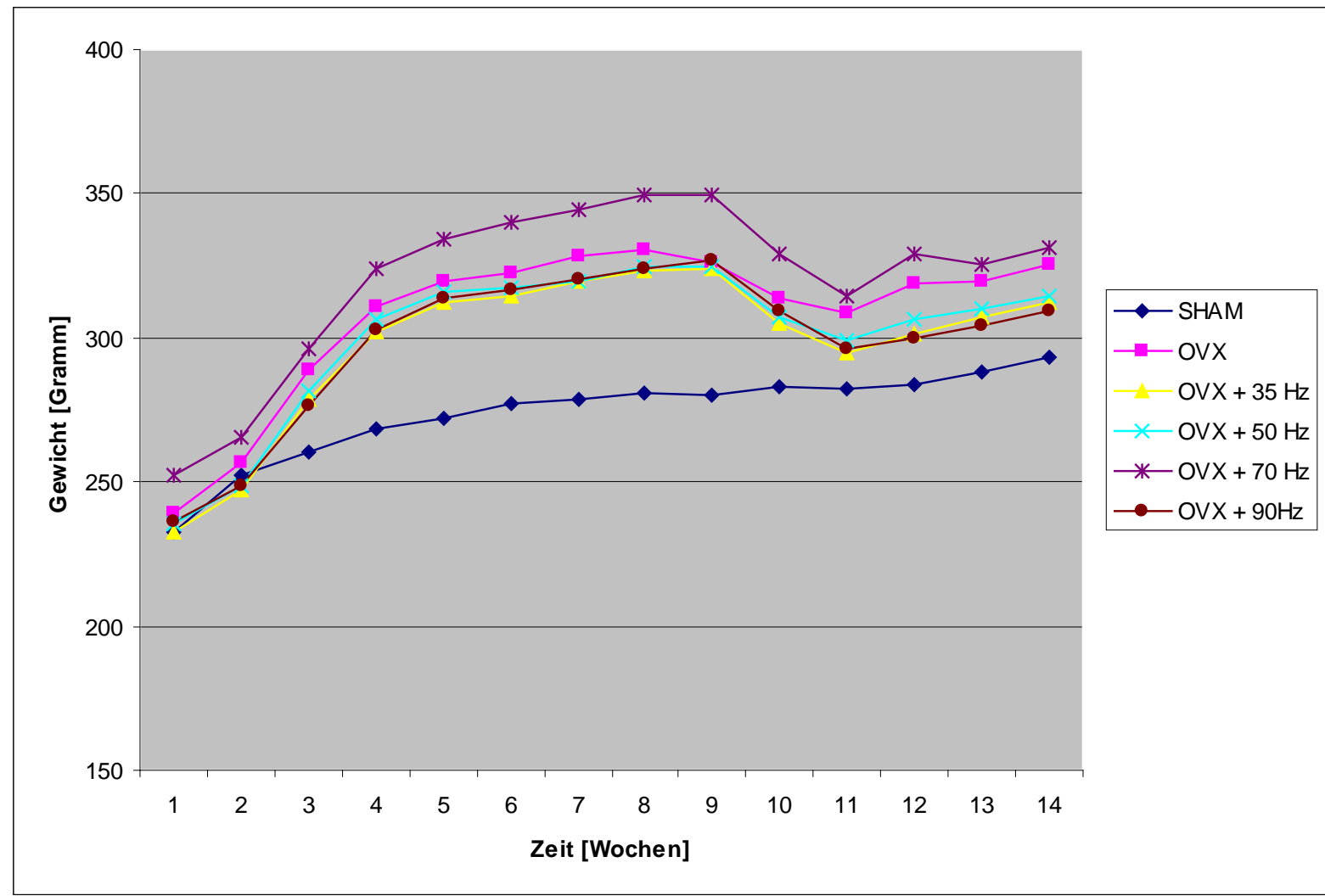

Abbildung 17: Durchschnittliches Körpergewicht der Tiere im Versuchsverlauf

Zu Beginn des Versuches bestehen keine signifikanten Unterschiede im durchschnittlichen Körpergewicht der Ratten der verschiedenen Versuchsgruppen. Während der ersten acht Versuchswochen steigt das Gewicht aller ovarektomierten Ratten deutlich stärker an als das der scheinoperierten, gesunden Tiere und erreicht 
schnell signifikant höhere Werte. Die unterschiedlichen Frequenzen bei der Vibrationsbehandlung haben dagegen keinen signifikanten Einfluss auf das Körpergewicht der Tiere, auch das Gewicht der vibrierten Ratten und das der nichtvibrierten Tiere der OVX-Gruppe unterscheidet sich nicht nennenswert. Nach der Osteotomie fällt das Körpergewicht der ovarektomierten Ratten zunächst auf signifikant niedrigere Werte, um sich gegen Ende des Versuches wieder dem Gewicht vor der Osteotomie anzunähern. Das Körpergewicht der SHAM-Tiere ändert sich nach der Osteotomie nicht signifikant bis zum Ende des Versuches.

Weder die Futteraufnahme noch das Körpergewicht der Tiere werden in der vorliegenden Untersuchung durch die Vibration beeinflusst, was als Indiz für eine gute Verträglichkeit der Vibrationsbehandlung gewertet werden kann.

\subsubsection{Uterusgewicht}

Tabelle 6: Uterusgewicht [mg] der scheinoperierten (SHAM), ovarektomierten (OVX) sowie ovarektomierten und mit verschiedenen Frequenzen vibrierten Tiere am Tag der Obduktion als Mittelwerte \pm Standardabweichungen

\begin{tabular}{|c||c|c|c|c|c|c|}
\hline & SHAM & OVX & $\begin{array}{c}\text { OVX } \\
+\mathbf{3 5 H z}\end{array}$ & $\begin{array}{c}\text { OVX } \\
\mathbf{+ 5 0 H z}\end{array}$ & $\begin{array}{c}\text { OVX } \\
\mathbf{+} \mathbf{7 0 H z}\end{array}$ & $\begin{array}{c}\text { OVX } \\
\mathbf{+ 9 0 H z}\end{array}$ \\
\hline \hline $\begin{array}{c}\text { Uterusgewicht } \\
{[\mathrm{mg}]}\end{array}$ & $\begin{array}{c}604,000 \\
\pm 192,27\end{array}$ & $\begin{array}{c}115,333 \\
\pm 15,98\end{array}$ & $\begin{array}{c}105,000 \\
\pm 19,51\end{array}$ & $\begin{array}{c}103,571 \\
\pm 14,99\end{array}$ & $\begin{array}{c}101,427 \\
\pm 17,03\end{array}$ & $\begin{array}{c}100,000 \\
\pm 11,09\end{array}$ \\
\hline
\end{tabular}

Tabelle 6 zeigt das durchschnittliche Uterusgewicht der Tiere zum Zeitpunkt der Obduktion. Wesentliche Unterschiede zwischen den ovarektomierten Versuchsgruppen finden sich nicht. Es wird aber deutlich, dass das Uterusgewicht aller ovarektomierten Ratten in etwa um den Faktor 6 und damit signifikant unter dem der hormonell intakten SHAM-Tiere liegt. 


\subsection{Röntgenbefunde}

\subsubsection{Tibiapseudarthrosen}

Bei der Röntgenbefundung anhand der unter 2.2.2 genannten Kriterien war zunächst die Qualität der Tibiaosteotomieheilung überprüft worden: Die Begutachtung der Röntgenaufnahmen in 2 Ebenen ergab dabei in 5 Fällen einen nicht vollständig kalzifizierten Frakturkallus im Sinne einer Pseudarthrose.

Anlässlich der anschließend im Rahmen des biomechanischen Biegetests durchgeführten Stabilitätsprüfung der Präparate (s. 2.3.2) konnten diese Pseudarthrosen bestätigt werden. Es fand sich außerdem in 6 weiteren Fällen ein manuell noch elastischer Frakturkallus, sodass sich die Gesamtzahl der nicht auswertbaren Tibiae auf 11 erhöhte.

Da ohne Konsolidierung der Osteotomie eine graphische Darstellung oder biomechanische Prüfung des knöchernen Kallus nicht sinnvoll erscheint, wurden diese 11 Präparate von allen weiteren Untersuchungen ausgeschlossen.

\subsubsection{Fibulafrakturen}

Als weiteres röntgenologisches Kriterium war die Integrität der Fibula begutachtet worden, wobei sich 16 Fibulafrakturen fanden. Da von einer vermehrten Instabilität und damit biomechanischen Beeinflussung der Osteotomieheilung durch diese zusätzlichen Frakturen auszugehen ist, wurden auch diese Präparate nicht weiter untersucht.

In 4 Fällen war eine Koinzidenz von Fibulafraktur und Tibiapseudarthrose zu beobachten, sodass insgesamt 23 Präparate von den weiteren Untersuchungen ausgeschlossen werden mussten.

Im Einzelnen wurden dadurch aus der SHAM-Gruppe 2, aus der OVX-Gruppe 5, aus der $35 \mathrm{~Hz}$ - sowie der $50 \mathrm{~Hz}-$ Gruppe jeweils 4, aus der $70 \mathrm{~Hz}-$ Gruppe 3 und aus der 90Hz-Gruppe 5 Präparate nicht in die Auswertung einbezogen.

\subsubsection{Infektzeichen}

Hinweise auf Infektionen fanden sich bei der röntgenologischen Befundung nicht. Auch im Rahmen der manuellen Untersuchung nach 2.3.2 waren keine Infektzeichen festzustellen. 


\subsection{Ergebnisse des biomechanischen Biegetests}

Mittelwerte und Standardabweichungen der im biomechanischen Biegetest ermittelten Ergebnisse für Steifheit und maximale Belastbarkeit des Kallus sind nachfolgend als Säulendiagramme dargestellt. Eine numerische Auflistung der einzelnen Werte findet sich zusätzlich in Tabelle 9 im tabellarischen Anhang.

\subsubsection{Steifheit des Kallus}

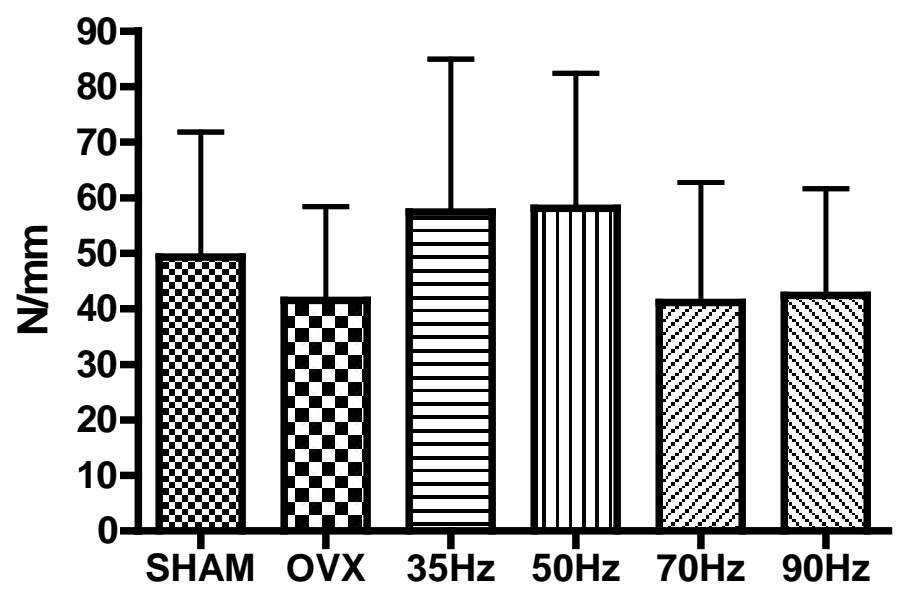

Abbildung 18: Steifheit des Tibiakallus repräsentiert durch die Steigung des Kurvenverlaufs im biomechanischen Biegetest

Dargestellt sind MW $\pm S D$, signifikante Unterschiede finden sich nicht $(p<0,05$, Tukey-Test)

Abbildung 18 stellt die Steigungen der im Biegetest ermittelten Kraft-WegDiagramme als Maß für die Steifheit bzw. Biegefestigkeit des Tibiakallus in den verschiedenen Versuchsgruppen dar. Signifikante Unterschiede zwischen den Gruppen finden sich nicht. Die Steifheit des Kallus ist aber bei der ovarektomierten OVX-Gruppe erkennbar geringer als bei der hormonell intakten SHAM-Gruppe. Unter allen Versuchsgruppen lassen die $50 \mathrm{~Hz}$ - und die $35 \mathrm{~Hz}-\mathrm{Gruppe}$ die tendenziell höchsten Werte für Steifheit bzw. Biegefestigkeit des Kallus erkennen. 


\subsubsection{Maximale Belastbarkeit des Kallus}

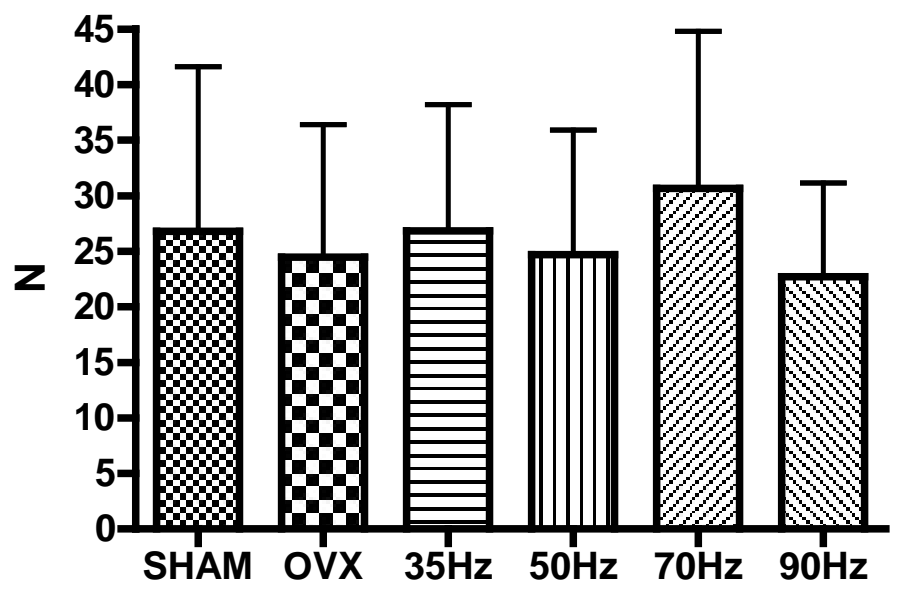

Abbildung 19: Maximale Belastbarkeit des Tibiakallus repräsentiert durch die im Kurvenverlauf des biomechanischen Biegetests ermittelte Streckgrenze Dargestellt sind $M W \pm S D$, signifikante Unterschiede finden sich nicht $(p<0,05$, Tukey-Test $)$

Die im Kurvenverlauf des biomechanischen Biegetests ermittelten Streckgrenzen (Yield Load) als Maß für die maximale Belastbarkeit des Tibiakallus zeigen keine signifikanten Unterschiede zwischen den einzelnen Versuchsgruppen. Die Werte der ovarektomierten OVX-Gruppe sind dabei etwas geringer als die der hormonell intakten SHAM-Gruppe. Die maximale Belastbarkeit des Kallus ist bei der $70 \mathrm{~Hz}$ Gruppe deutlich größer als bei allen anderen Untersuchungsgruppen und damit auch größer als bei der nichtovarektomierten SHAM-Gruppe. 


\subsection{Ergebnisse der Micro-CT-Untersuchung}

Im Folgenden sind Mittelwerte und Standardabweichungen der einzelnen Micro-CTErgebnisse in Form von Säulendiagrammen dargestellt. Soweit sich signifikante Unterschiede zwischen den Versuchsgruppen $(p<0,05)$ finden, werden diese durch unterschiedliche Buchstaben über den Säulen kenntlich gemacht. Die numerischen Werte der Messparameter sind wieder zusätzlich in Tabelle $10 \mathrm{im}$ tabellarischen Anhang aufgeführt.

\subsubsection{Gesamtknochendichte}

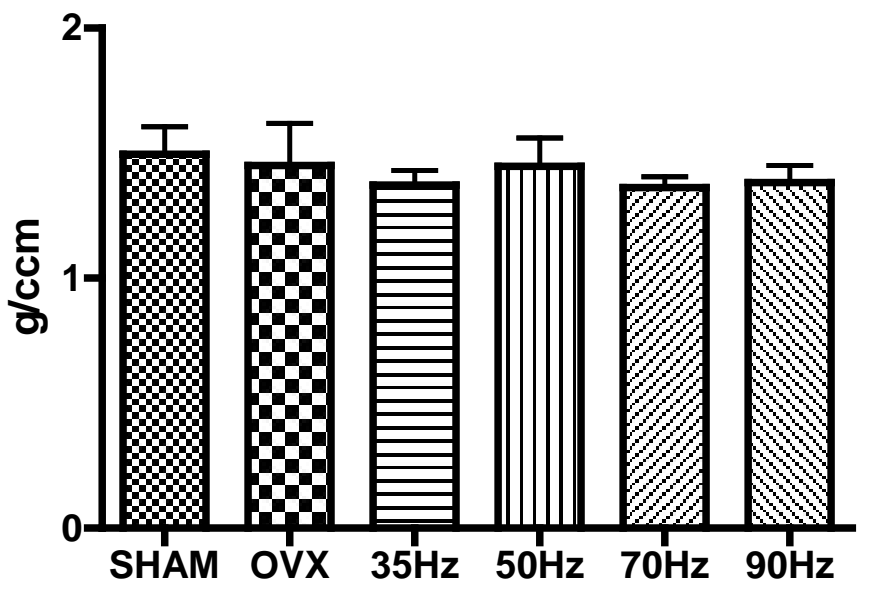

Abbildung 20: Micro-CT-Messparameter „Gesamtknochendichte“ Dargestellt sind $M W \pm S D$, signifikante Unterschiede finden sich nicht $(p<0,05$, Tukey-Test)

Die Gesamtknochendichte der OVX-Gruppe liegt nur geringfügig unter der der SHAM-Gruppe. Durch die Vibrationsbehandlung lässt sich keine Dichtezunahme gegenüber der OVX-Gruppe erreichen. Die Gesamtknochendichte der $50 \mathrm{~Hz}-\mathrm{Gruppe}$ liegt geringfügig über der der anderen Vibrationsgruppen, wesentliche oder gar signifikante Unterschiede zwischen den einzelnen Gruppen finden sich nicht. 


\subsubsection{Kortikalisdichte}

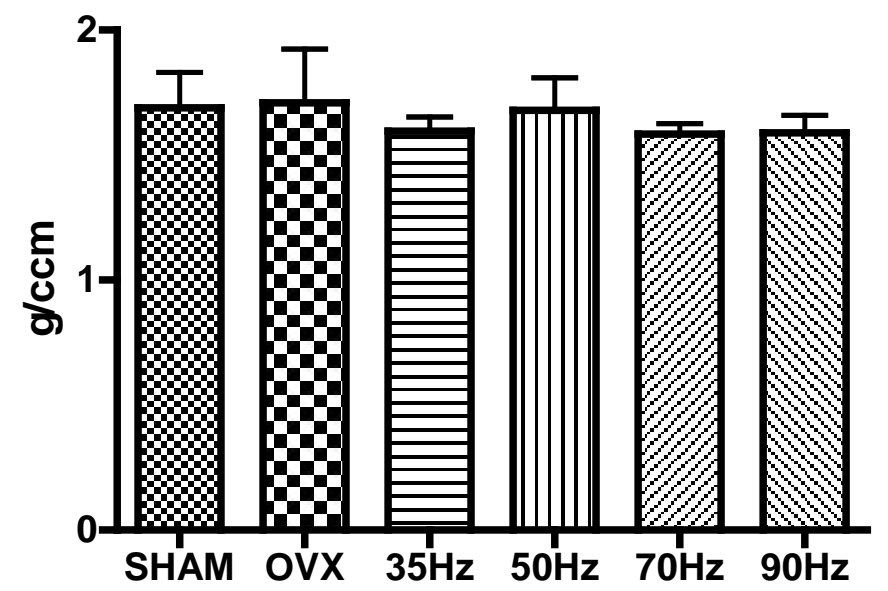

Abbildung 21: Micro-CT-Messparameter „Kortikalisdichte“

Dargestellt sind $M W \pm S D$, signifikante Unterschiede finden sich nicht $(p<0,05$, Tukey-Test $)$

Die Kortikalisdichte der hormonell intakten SHAM-Gruppe und die der ovarektomierten OVX-Gruppe unterscheiden sich nicht nennenswert. Die Kortikalisdichte der $50 \mathrm{~Hz}-G r u p p e$ übertrifft die der anderen Vibrationsgruppen geringfügig. Signifikante Unterschiede zwischen den einzelnen Gruppen finden sich nicht. 


\subsubsection{Kallusdichte}

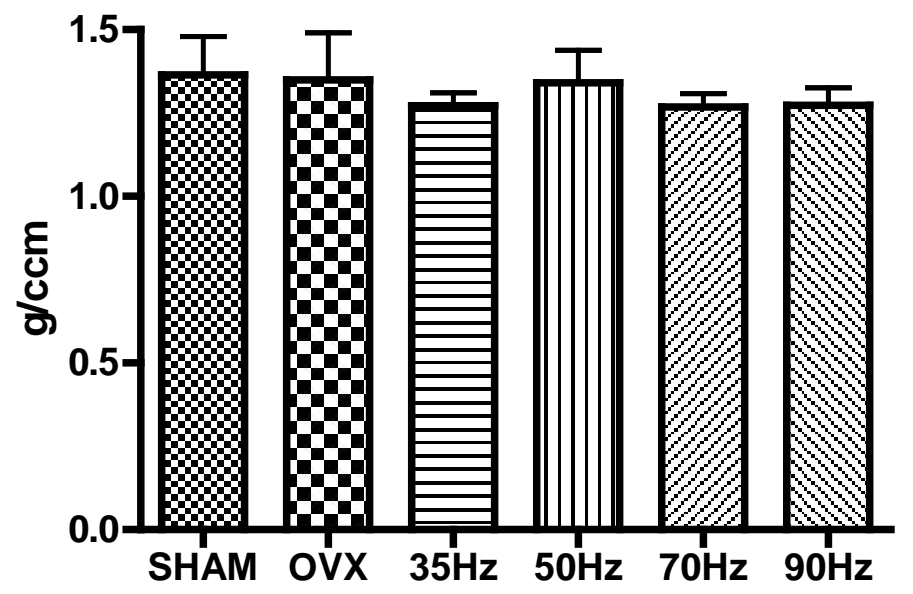

Abbildung 22: Micro-CT-Messparameter „Kallusdichte“

Dargestellt sind $M W \pm S D$, signifikante Unterschiede finden sich nicht $(p<0,05$, Tukey-Test)

Auch die Kallusdichte unterscheidet sich zwischen den einzelnen Versuchsgruppen nicht wesentlich. Die Kallusdichte der 50Hz-Gruppe übertrifft die der anderen Vibrationsgruppen geringfügig. 


\subsubsection{Gesamtknochenvolumen}

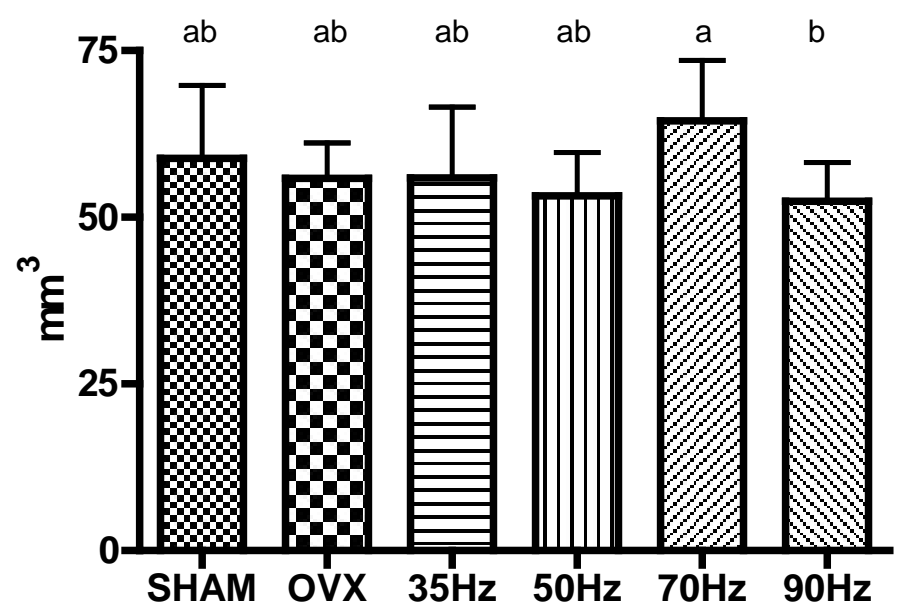

Abbildung 23: Micro-CT-Messparameter „Gesamtknochenvolumen“

Dargestellt sind MW \pm SD, MW mit verschiedenen Buchstaben unterscheiden sich signifikant ( $p<0,05$, Tukey-Test)

Das Gesamtknochenvolumen der ovarektomierten OVX-Gruppe liegt geringfügig unter dem der hormonell intakten SHAM-Gruppe. Die $70 \mathrm{~Hz}-$ Vibration hat als einzige Vibrationsfrequenz eine deutliche Zunahme des Gesamtknochenvolumens zur Folge. Das Gesamtknochenvolumen der $70 \mathrm{~Hz}$-Gruppe übertrifft das aller anderen Versuchsgruppen, die Differenz ist aber nur gegenüber der $90 \mathrm{~Hz}-$ Gruppe signifikant, die das kleinste Gesamtknochenvolumen aller Gruppen aufweist. 


\subsubsection{Kortikalisvolumen}

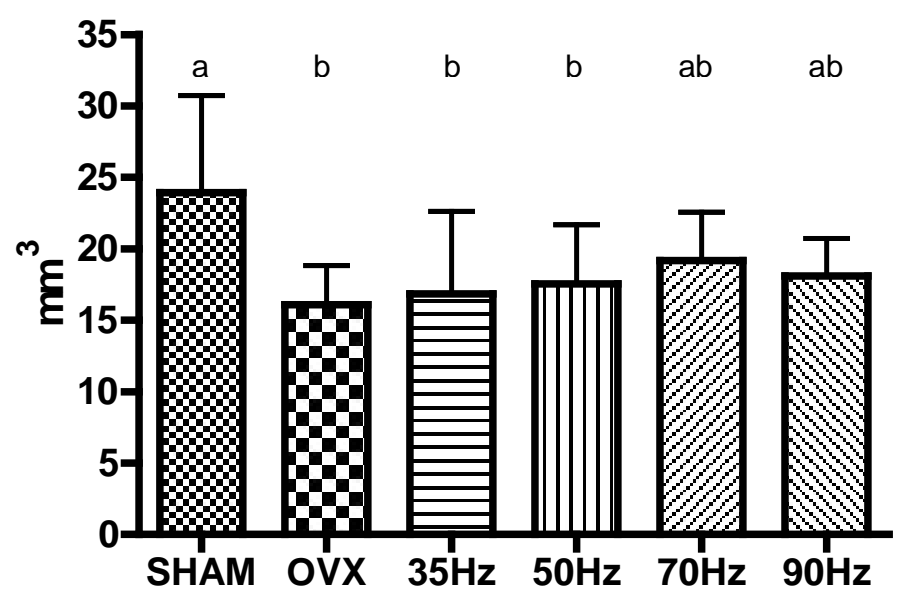

Abbildung 24: Micro-CT-Messparameter „Kortikalisvolumen“

Dargestellt sind MW \pm SD, MW mit verschiedenen Buchstaben unterscheiden sich signifikant $(p<0,05$, Tukey-Test $)$

Das Kortikalisvolumen der SHAM-Gruppe liegt signifikant über dem der OVX-Gruppe und dem der $35 \mathrm{~Hz}$ - und der $50 \mathrm{~Hz}$-Gruppe. Im Vergleich zur nichtvibrierten OVXGruppe hat die Vibrationsbehandlung mit allen Frequenzen eine Zunahme des Kortikalisvolumens zur Folge. Das größte Kortikalisvolumen aller ovarektomierten Gruppen wird unter der Vibrationsfrequenz von $70 \mathrm{~Hz}$ erreicht. 


\subsubsection{Kallusvolumen}

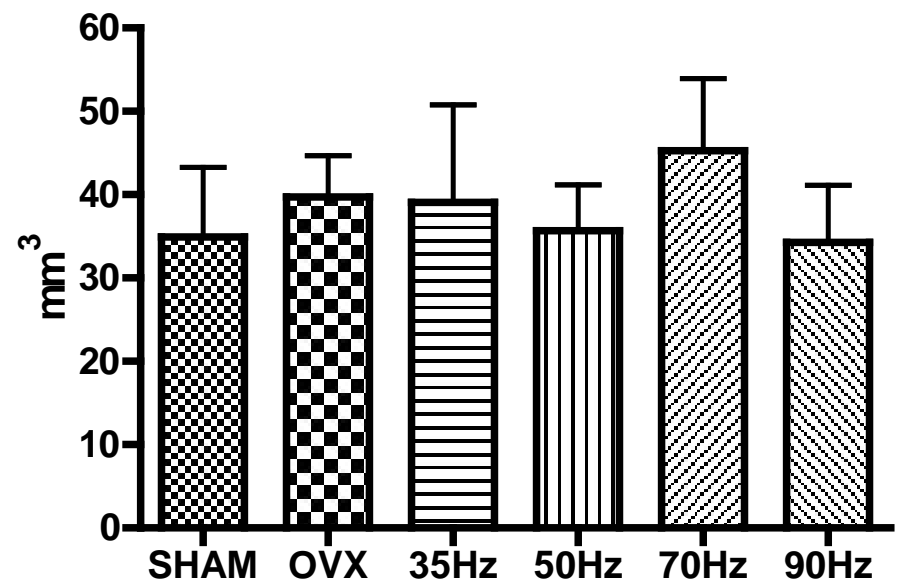

Abbildung 25: Micro-CT-Messparameter „Kallusvolumen“

Dargestellt sind $M W \pm S D$, signifikante Unterschiede finden sich nicht $(p<0,05$, Tukey-Test)

Das Kallusvolumen der hormonell intakten SHAM-Gruppe ist etwas kleiner als das der ovarektomierten OVX-Gruppe und auch kleiner als das der $35 \mathrm{~Hz}-$, der $50 \mathrm{~Hz}-$ und der $70 \mathrm{~Hz}-G r u p p e$, es wird nur vom Wert der $90 \mathrm{~Hz}-$ Gruppe unterschritten. Das Kallusvolumen der $70 \mathrm{~Hz}-G r u p p e$ ist deutlich größer als das aller anderen Gruppen, die Differenz ist aber nicht signifikant. 


\subsubsection{Bone volume fraction}

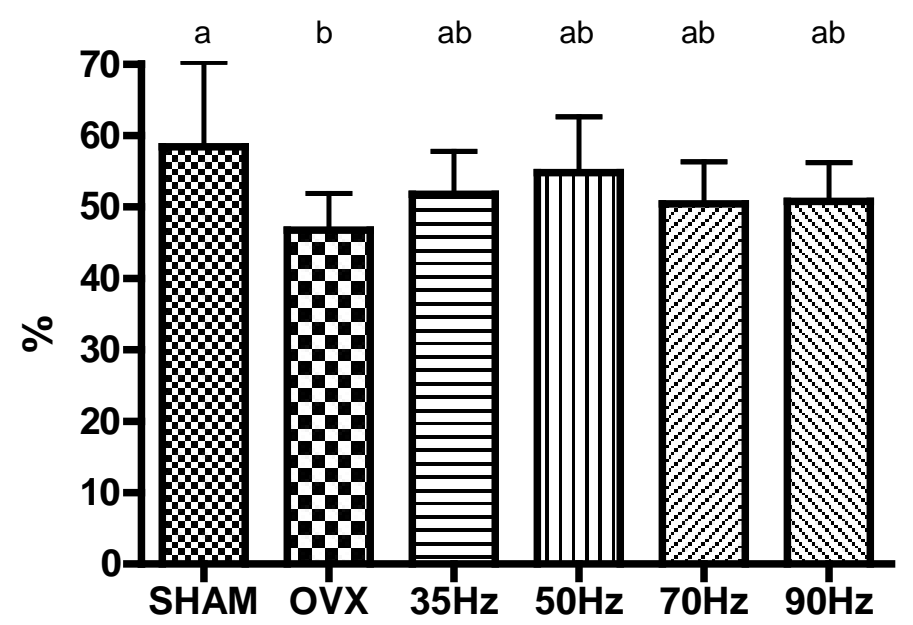

Abbildung 26: Micro-CT-Messparameter „bone volume fraction“

Dargestellt sind MW $\pm S D, M W$ mit verschiedenen Buchstaben unterscheiden sich signifikant $(p<0,05$, Tukey-Test)

Die „bone volume fraction“ bzw. der Knochenanteil am Gesamtgewebevolumen ist bei der OVX-Gruppe infolge der Ovarektomie signifikant kleiner als bei der hormonell intakten SHAM-Gruppe. Durch alle Vibrationsfrequenzen kann die „bone volume fraction" gegenüber der nichtvibrierten OVX-Gruppe tendenziell, aber nicht signifikant gesteigert werden. Die relativ höchsten Messwerte aller Vibrationsgruppen werden unter der Frequenz von $50 \mathrm{~Hz}$ erreicht. 


\subsection{Ergebnisse der Mikroradiographie}

Nachfolgend sind Mittelwerte und Standardabweichungen der mikroradiographisch ermittelten Messparameter in Form von Säulendiagrammen dargestellt, wobei signifikante Unterschiede $(p<0,05)$ zwischen den einzelnen Gruppen wieder durch unterschiedliche Buchstaben über den Säulen hervorgehoben werden. Alle Werte finden sich zusätzlich als Zahlen in Tabelle 11 im tabellarischen Anhang.

\subsubsection{Kortikalisdicke ventromedial}

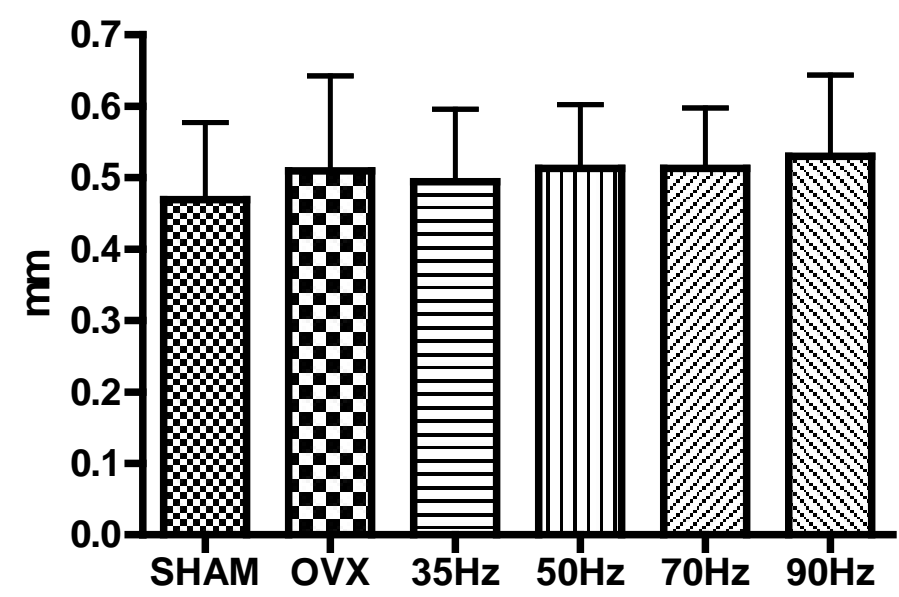

Abbildung 27: Mikroradiographisch ermittelter Messparameter „Kortikalisdicke ventromedial“

Dargestellt sind $M W \pm S D$, signifikante Unterschiede finden sich nicht $(p<0,05$, Tukey-Test)

Die ventromediale Kortikalis ist bei den Tieren aller Versuchsgruppen annähernd gleich dick, signifikante Unterschiede zwischen den einzelnen Gruppen bestehen nicht. Die ventromediale Kortikalis der hormonell intakten SHAM-Ratten ist geringfügig dünner als die der ovarektomierten Tiere. 


\subsubsection{Kortikalisdicke dorsal}

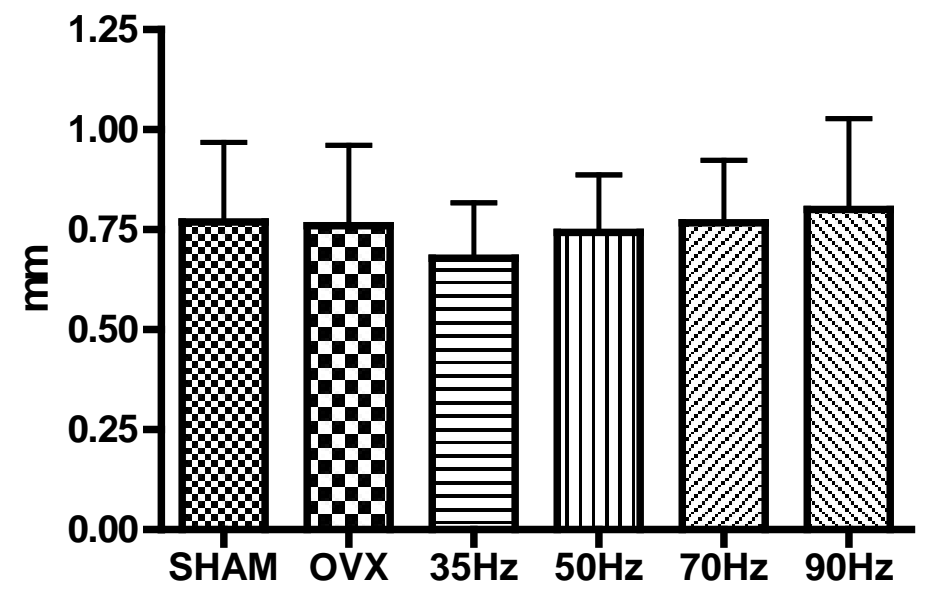

Abbildung 28: Mikroradiographisch ermittelter Messparameter „Kortikalisdicke dorsal“

Dargestellt sind MW $\pm S D$, signifikante Unterschiede finden sich nicht $(p<0,05$, Tukey-Test)

Auch bei der dorsalen Kortikalisdicke zeigen sich keine signifikanten Unterschiede zwischen den Versuchsgruppen. 


\subsubsection{Kallusdicke ventromedial}

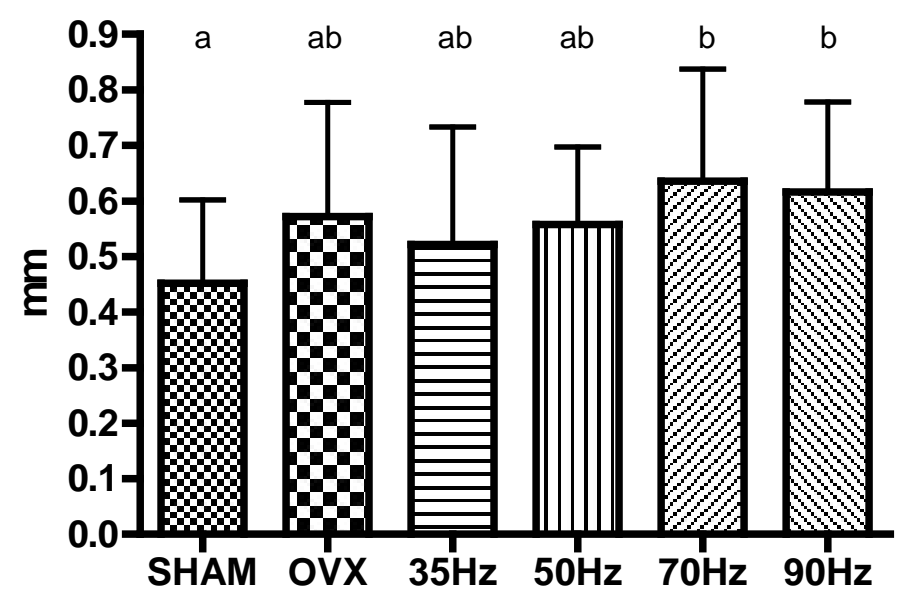

Abbildung 29: Mikroradiographisch ermittelter Messparameter „Kallusdicke ventromedial“

Dargestellt sind MW \pm SD, MW mit verschiedenen Buchstaben unterscheiden sich signifikant $(p<0,05$, Tukey-Test)

Bei Betrachtung der ventromedialen Kallusdicke fällt auf, dass diese bei allen ovarektomierten Versuchsgruppen größer ist als bei den hormonell intakten SHAMTieren. Ein signifikanter Unterschied besteht allerdings nur zwischen SHAM-Gruppe und $70 \mathrm{~Hz}$ - bzw. $90 \mathrm{~Hz}-G r u p p e$, die den dicksten ventromedialen Kallus aller Versuchsgruppen aufweisen. 


\subsubsection{Kallusdicke dorsal}

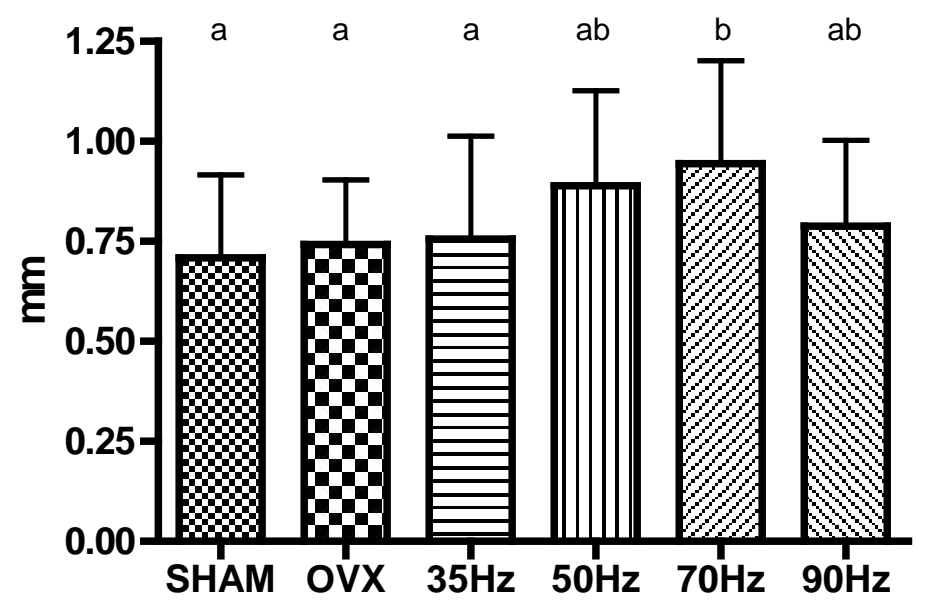

Abbildung 30: Mikroradiographisch ermittelter Messparameter „Kallusdicke dorsal“

Dargestellt sind MW $\pm S D, M W$ mit verschiedenen Buchstaben unterscheiden sich signifikant $(p<0,05$, Tukey-Test $)$

Die dorsale Kallusdicke aller ovarektomierten Gruppen übertrifft die der nichtovarektomierten SHAM-Gruppe zumindest geringfügig. Die höchsten Werte erreicht die dorsale Kallusdicke bei der $70 \mathrm{~Hz}-G$ ruppe mit signifikantem Unterschied zur SHAM-Gruppe, aber auch zur OVX- und zur 35Hz-Gruppe. 


\subsubsection{Kortikalisdichte ventromedial}

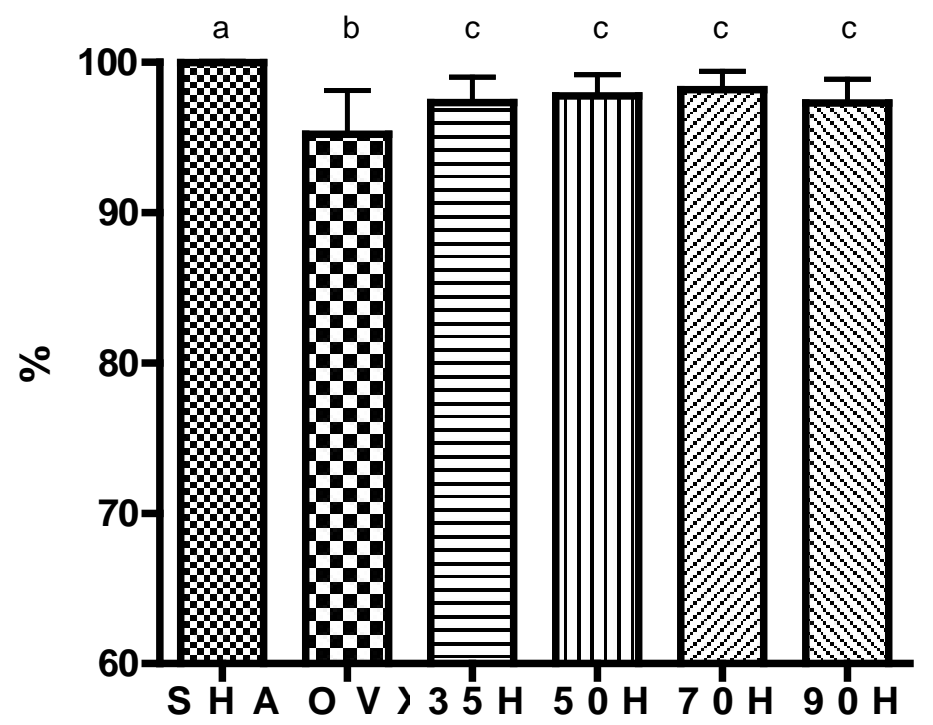

Abbildung 31: Mikroradiographisch ermittelter Messparameter „Kortikalisdichte ventromedial“

Dargestellt sind MW $\pm S D, M W$ mit verschiedenen Buchstaben unterscheiden sich signifikant $(p<0,05$, Tukey-Test)

Die ventromediale Kortikalisdichte ist bei der OVX-Gruppe signifikant kleiner als bei der hormonell intakten SHAM-Gruppe. Auch alle Vibrationsgruppen erreichen eine signifikant höhere ventromediale Kortikalisdichte als die nichtvibrierte OVX-Gruppe. 


\subsubsection{Kortikalisdichte dorsal}

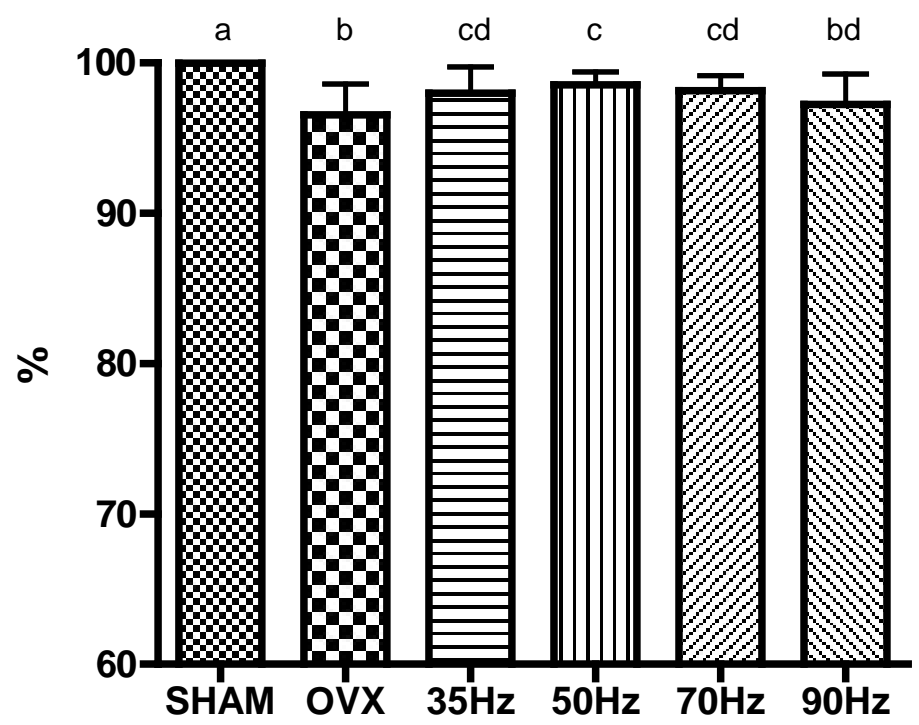

\section{Abbildung 32: Mikroradiographisch ermittelter Messparameter „Kortikalisdichte dorsal“}

Dargestellt sind MW $\pm S D, M W$ mit verschiedenen Buchstaben unterscheiden sich signifikant $(p<0,05$, Tukey-Test)

Die dorsale Kortikalisdichte ist bei allen ovarektomierten Gruppen signifikant kleiner als bei der hormonell intakten SHAM-Gruppe. Im Vergleich zur nichtvibrierten OVXGruppe hat die Vibration bei allen Gruppen eine Zunahme der dorsalen Kortikalisdichte zur Folge. Diese Zunahme ist für die $35 \mathrm{~Hz}-$, die $50 \mathrm{~Hz}-$ und die $70 \mathrm{~Hz}-\mathrm{Gruppe}$ signifikant, nicht dagegen für die $90 \mathrm{~Hz}$-Gruppe, die von allen Vibrationsgruppen die geringste dorsale Kortikalisdichte erreicht. Die Werte der $90 \mathrm{~Hz}-G r u p p e$ sind signifikant kleiner als die der $50 \mathrm{~Hz}-$ Gruppe. 


\subsubsection{Kallusdichte ventromedial}

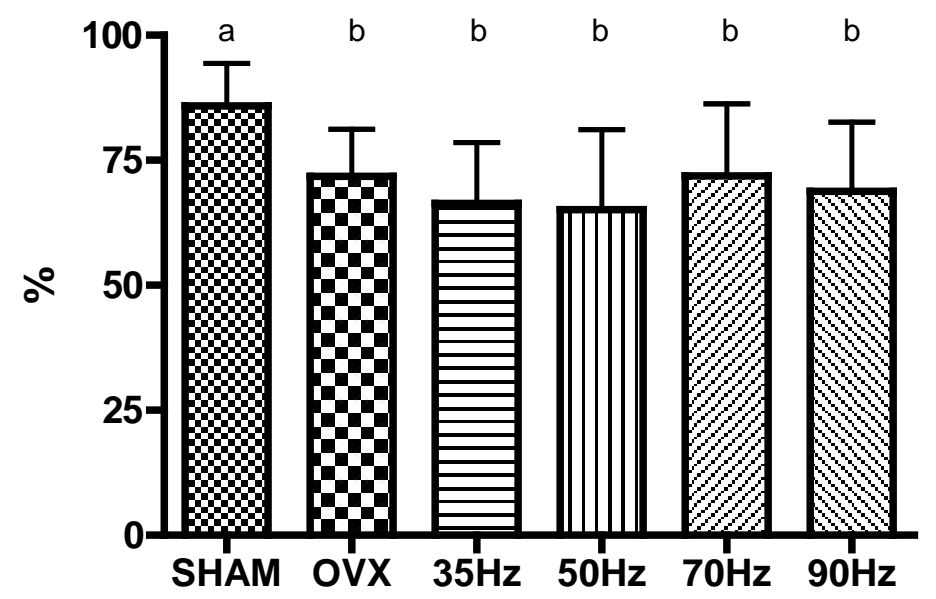

\section{Abbildung 33: Mikroradiographisch ermittelter Messparameter} „Kallusdichte ventromedial“

Dargestellt sind MW $\pm S D, M W$ mit verschiedenen Buchstaben unterscheiden sich signifikant $(p<0,05$, Tukey-Test)

Die ventromediale Kallusdichte der SHAM-Gruppe ist signifikant größer als die der anderen, ovarektomierten Versuchsgruppen. Weitere signifikante Differenzen finden sich zwischen den einzelnen Gruppen nicht. Die ventromediale Kallusdichte ist unter der $70 \mathrm{~Hz}$-Vibration aber tendenziell größer als unter der Vibration mit anderen Frequenzen und auch größer als bei der nichtvibrierten OVX-Gruppe. 


\subsubsection{Kallusdichte dorsal}

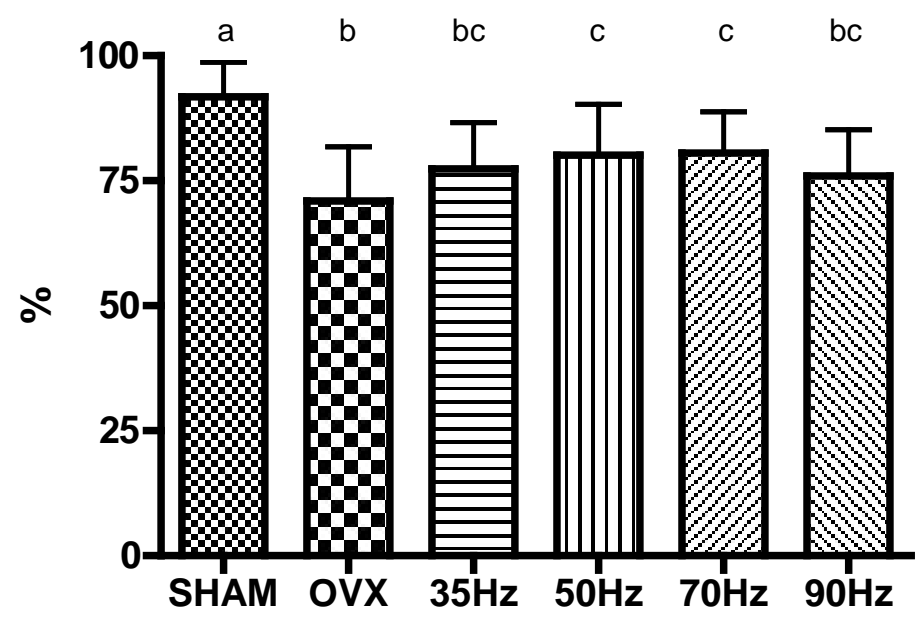

\section{Abbildung 34: Mikroradiographisch ermittelter Messparameter} „Kallusdichte dorsal“

Dargestellt sind MW $\pm S D, M W$ mit verschiedenen Buchstaben unterscheiden sich signifikant $(p<0,05$, Tukey-Test)

Auch die dorsale Kallusdichte der hormonell intakten SHAM-Gruppe liegt signifikant über der aller übrigen, ovarektomierten Versuchsgruppen. Im Vergleich zur nichtvibrierten OVX-Gruppe hat die Vibrationsbehandlung bei allen Gruppen eine Zunahme der dorsalen Kallusdichte zur Folge. Diese Dichtezunahme ist für die $50 \mathrm{~Hz}$ - und die $70 \mathrm{~Hz}-$ Gruppe auch signifikant. 


\subsubsection{Kallusdichte endostal}

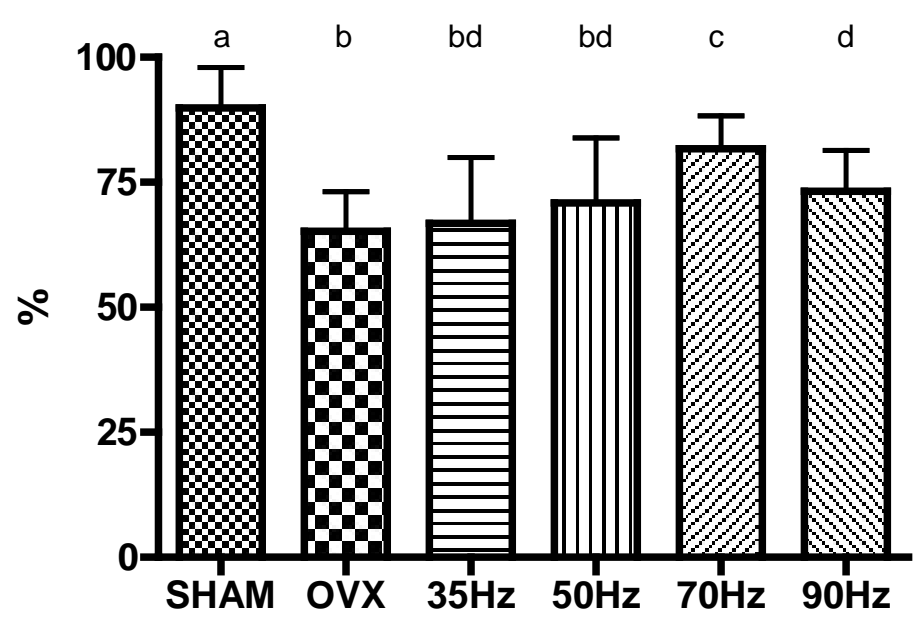

\section{Abbildung 35: Mikroradiographisch ermittelter Messparameter „Kallusdichte endostal“}

Dargestellt sind MW \pm SD, MW mit verschiedenen Buchstaben unterscheiden sich signifikant $(p<0,05$, Tukey-Test $)$

Die endostale Kallusdichte der SHAM-Gruppe ist signifikant höher als die aller anderen, ovarektomierten Versuchsgruppen. Beim Vergleich der Vibrationsgruppen mit der OVX-Gruppe fallen die $70 \mathrm{~Hz}$ - und die $90 \mathrm{~Hz}-$-Gruppe durch signifikant größere Messwerte auf. Die anderen Vibrationsgruppen weisen ebenfalls eine höhere endostale Kallusdichte als die OVX-Gruppe auf, die Differenz ist hier allerdings nicht signifikant. Die $70 \mathrm{~Hz}-$ Gruppe, als die Vibrationsgruppe mit der höchsten endostalen Kallusdichte, erreicht auch signifikant größere Dichtewerte als alle übrigen Vibrationsgruppen. 


\subsubsection{Trabekelflächendichte}

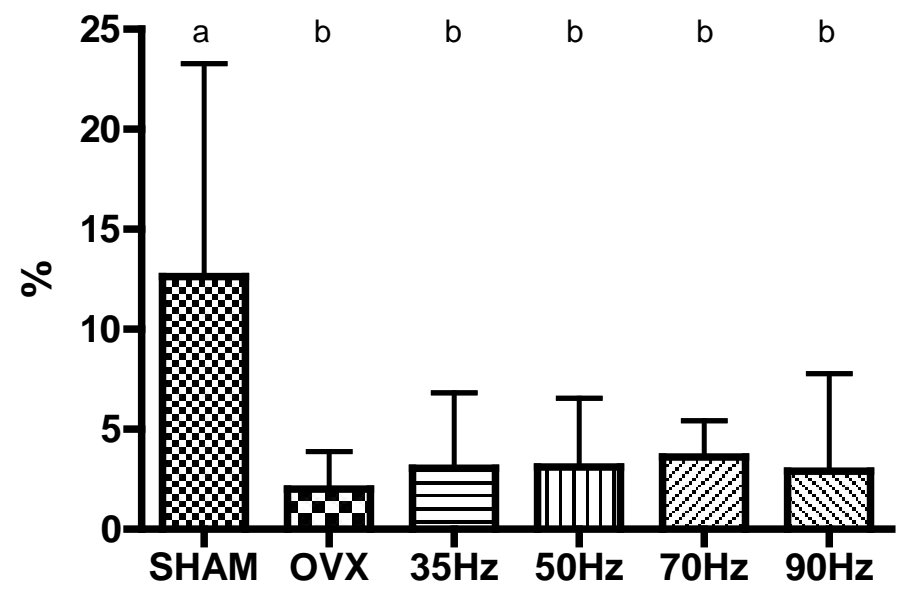

\section{Abbildung 36: Mikroradiographisch ermittelter Messparameter „Trabekelflächendichte“}

Dargestellt sind MW $\pm S D, M W$ mit verschiedenen Buchstaben unterscheiden sich signifikant $(p<0,05$, Tukey-Test $)$

Die SHAM-Gruppe weist eine signifikant höhere Trabekelflächendichte auf als alle anderen, ovarektomierten Versuchsgruppen. Im Vergleich zur nichtvibrierten OVXGruppe lassen alle Vibrationsgruppen eine Tendenz zur Verbesserung der Trabekelstruktur erkennen. Die $70 \mathrm{~Hz}-$ Gruppe erreicht unter allen Vibrationsgruppen die höchsten Werte. 


\subsubsection{Anzahl Trabekelkreuzungen absolut}

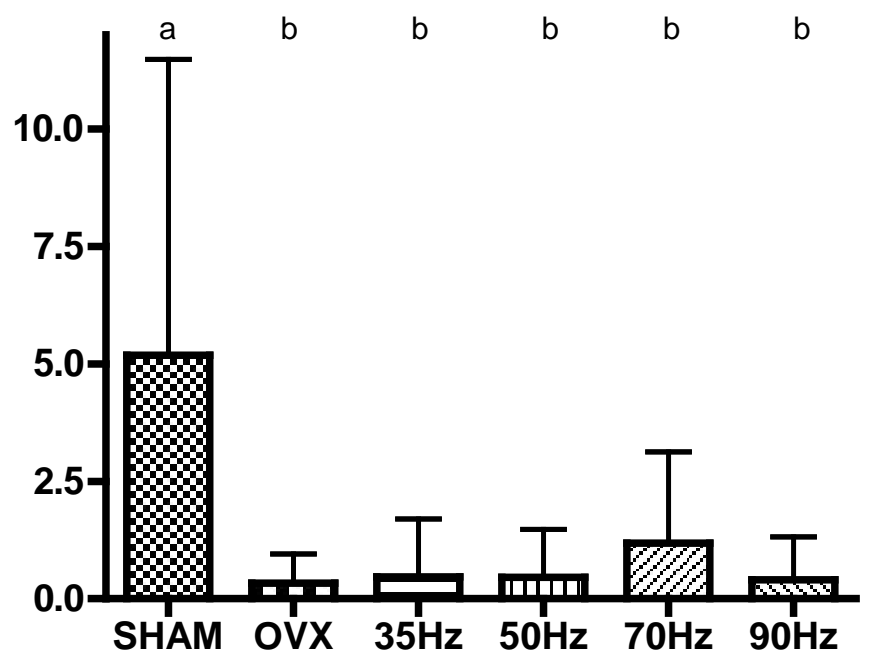

\footnotetext{
Abbildung 37: Mikroradiographisch ermittelter Messparameter „Anzahl Trabekelkreuzungen absolut"

Dargestellt sind MW $\pm S D, M W$ mit verschiedenen Buchstaben unterscheiden sich signifikant $(p<0,05$, Tukey-Test $)$
}

Auch die absolute Anzahl an Trabekelkreuzungen ist bei allen ovarektomierten Versuchsgruppen signifikant kleiner als bei der hormonell intakten SHAM-Gruppe. Unter den Vibrationsgruppen hat nur die $70 \mathrm{~Hz}-$ Vibration eine deutliche Zunahme der Trabekelkreuzungszahl zur Folge. Die Differenz ist gegenüber den anderen Vibrationsgruppen deutlich, aber nicht signifikant. 


\subsubsection{Anzahl Trabekelkreuzungen pro $\mathrm{mm}^{2}$}

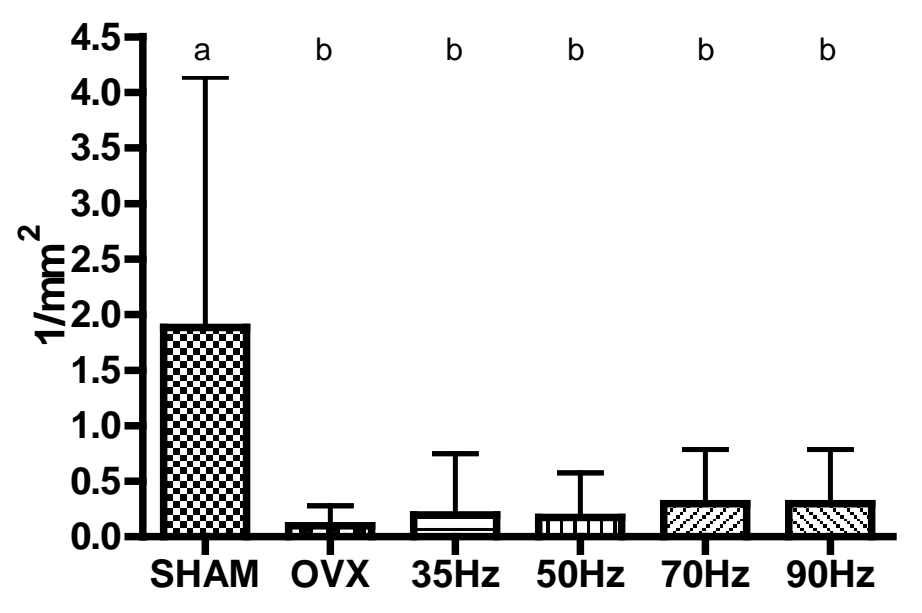

Abbildung 38: Mikroradiographisch ermittelter Messparameter „Anzahl Trabekelkreuzungen pro $\mathrm{mm}^{2}$ “

Dargestellt sind MW $\pm S D, M W$ mit verschiedenen Buchstaben unterscheiden sich signifikant $(p<0,05$, Tukey-Test)

Bei der hormonell intakten SHAM-Gruppe finden sich pro $\mathrm{mm}^{2}$ signifikant mehr Trabekelkreuzungen als bei allen anderen, ovarektomierten Versuchsgruppen. Unter allen ovarektomierten Gruppen ist die Anzahl der Trabekelkreuzungen pro $\mathrm{mm}^{2}$ bei der $70 \mathrm{~Hz}$ - und der $90 \mathrm{~Hz}-$ Gruppe tendenziell am größten. 


\subsubsection{Mittlere Trabekeldicke}

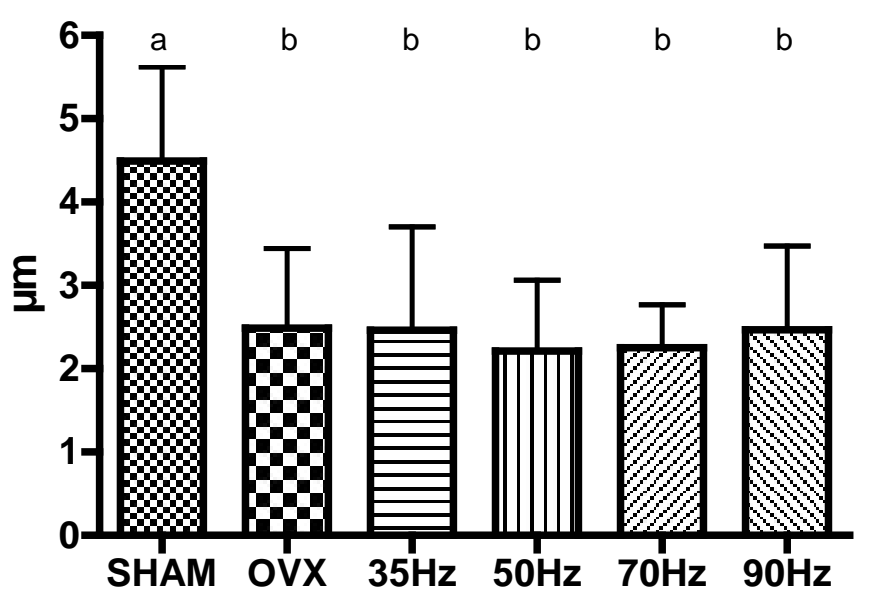

Abbildung 39: Mikroradiographisch ermittelter Messparameter „Mittlere Trabekeldicke“

Dargestellt sind MW $\pm S D, M W$ mit verschiedenen Buchstaben unterscheiden sich signifikant $(p<0,05$, Tukey-Test $)$

Wie bei den anderen Trabekelparametern ist auch die mittlere Trabekeldicke der hormonell intakten SHAM-Gruppe signifikant größer als die aller übrigen, ovarektomierten Versuchsgruppen. Ansonsten variiert die mittlere Trabekeldicke der einzelnen Gruppen nur gering. 


\subsection{Ergebnisse der polychromen Sequenzmarkierung}

Im Folgenden sind die verschiedenen fluoreszenzmikroskopisch definierten Kallusregionen (ventromedial, dorsal und endostal) unter dem Einfluss der unterschiedlichen Fluorochrome in Form von Säulendiagrammen dargestellt. Die durch Summation der einzelnen fluorochrommarkierten Areale ermittelte Gesamtkallusfläche einer jeden Region wird den Fluorochromdiagrammen jeweils vorangestellt.

In den Diagrammen dargestellt sind wieder Mittelwerte und Standardabweichungen, signifikante Unterschiede $(p<0,05)$ zwischen den einzelnen Gruppen werden durch verschiedene Buchstaben über den Säulen hervorgehoben. Alle Werte sind zusätzlich als Zahlen in Tabelle 12 im tabellarischen Anhang aufgeführt.

\subsubsection{Kallusfläche ventromedial}

\subsubsection{Gesamtfläche Kallus ventromedial}

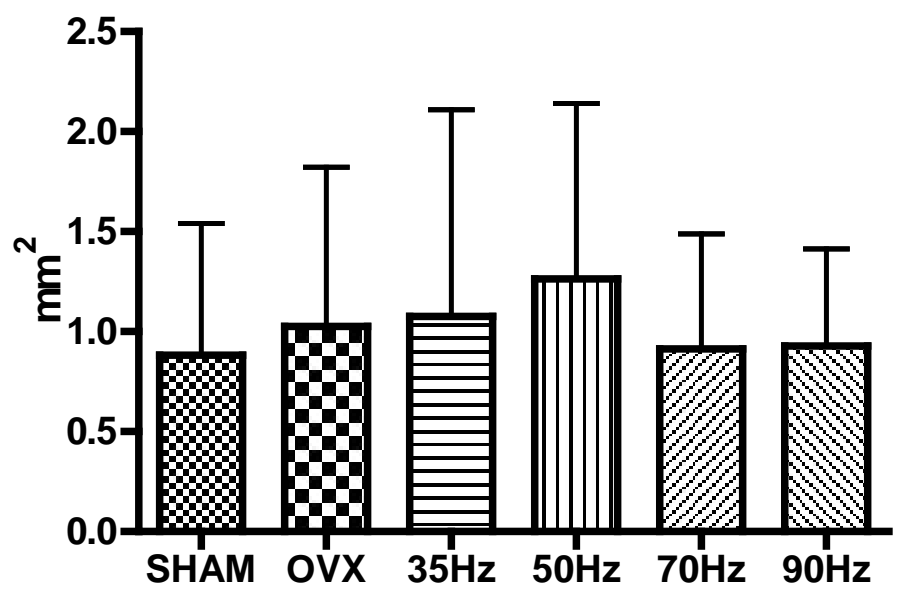

Abbildung 40: Fluoreszenzmikroskopisch ermittelter Messparameter "Gesamtfläche Kallus ventromedial“

Dargestellt sind $M W \pm S D$, signifikante Unterschiede finden sich nicht $(p<0,05$, Tukey-Test) 


\subsubsection{CG-Fläche Kallus ventromedial}

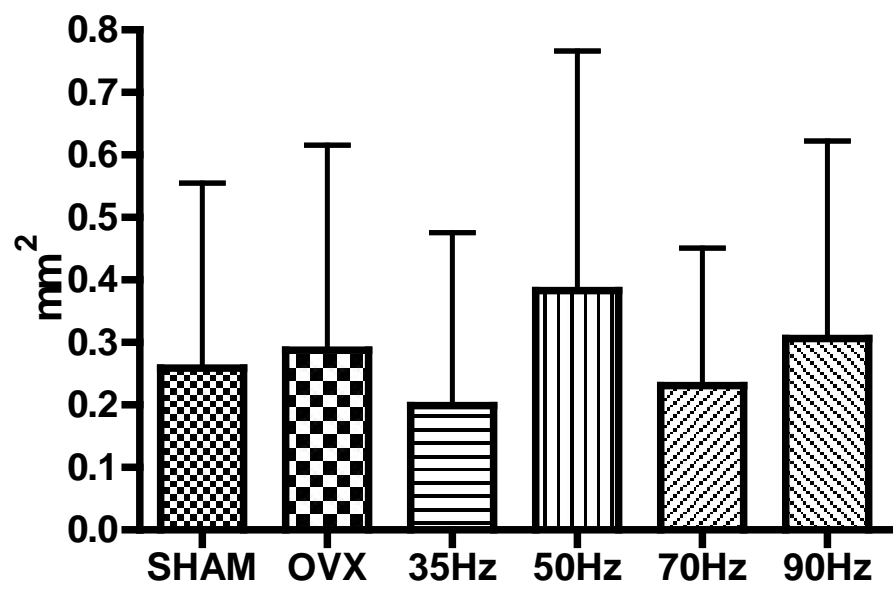

Abbildung 41: Fluoreszenzmikroskopisch ermittelter Messparameter „CG-Fläche Kallus ventromedial“"

Dargestellt sind MW $\pm S D$, signifikante Unterschiede finden sich nicht $(p<0,05$, Tukey-Test)

\subsubsection{AK-Fläche Kallus ventromedial}

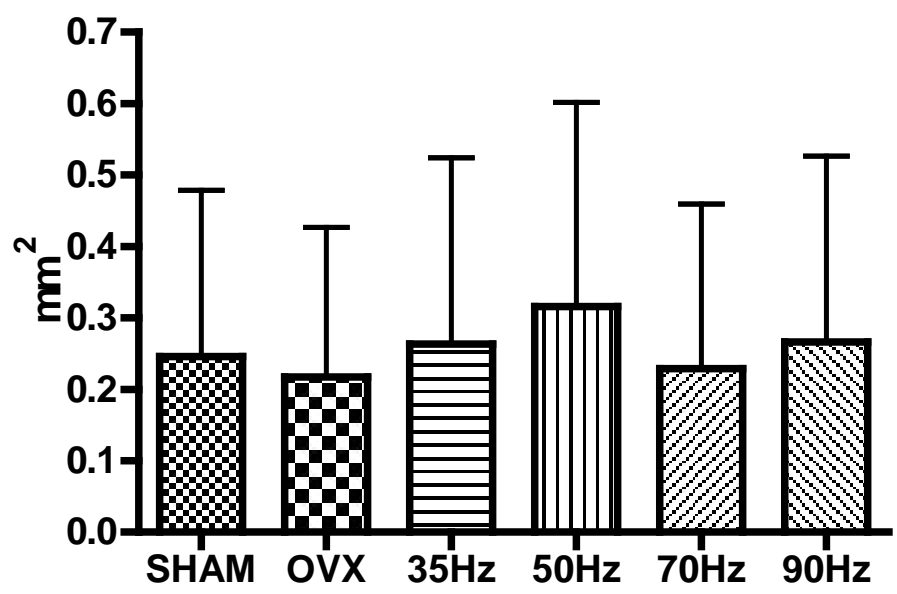

Abbildung 42: Fluoreszenzmikroskopisch ermittelter Messparameter „AK-Fläche Kallus ventromedial“

Dargestellt sind MW $\pm S D$, signifikante Unterschiede finden sich nicht $(p<0,05$, Tukey-Test) 


\subsubsection{TC-Fläche Kallus ventromedial}

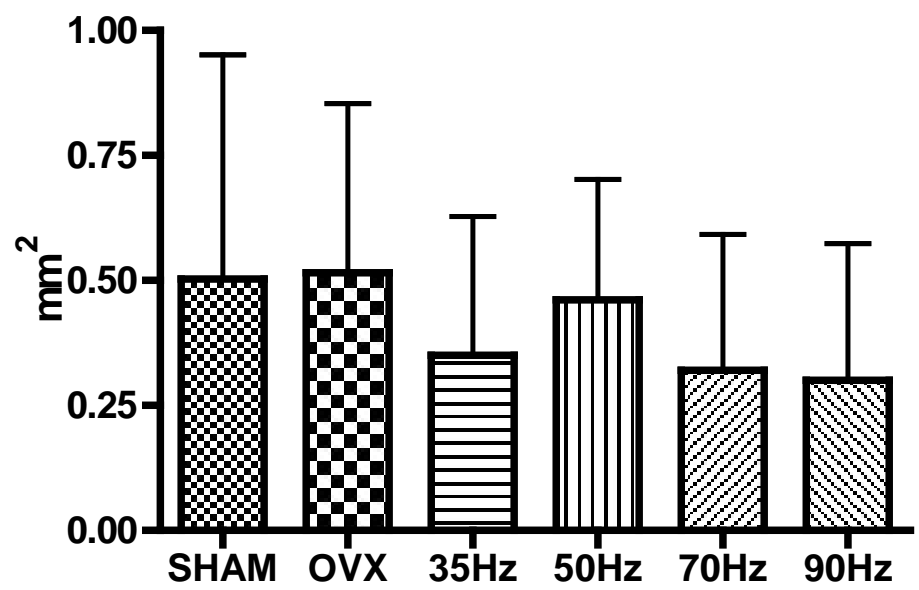

\section{Abbildung 43: Fluoreszenzmikroskopisch ermittelter Messparameter „TC-Fläche Kallus ventromedial"}

Dargestellt sind $M W \pm S D$, signifikante Unterschiede finden sich nicht $(p<0,05$, Tukey-Test $)$

Bei Auswertung der fluoreszenzmikroskopischen Bilder fand sich erwartungsgemäß in allen Fällen eine knöchernde Überbrückung des Osteotomiespalts. Der Umstand, dass sich bei allen Gruppen nur kleinste XO-markierte Areale erkennen ließen (s. 2.6.4.2), lässt dabei auf eine im Wesentlichen nach dem 13.Tag nach Osteotomie erfolgende messbare Kalzifizierung schließen.

Die fluoreszenzmikroskopische Analyse der ventromedialen Kallusflächen lässt aufgrund der hohen Standardabweichungen keine signifikanten Unterschiede zwischen den verschiedenen Therapiegruppen erkennen. Ventromediale Gesamtkallusfläche und ventromediale Kallusfläche im CG- und AK-Intervall sind aber bei der $50 \mathrm{~Hz}$-Therapiegruppe tendenziell größer als bei allen anderen Gruppen. 


\subsubsection{Kallusfläche dorsal}

\subsubsection{Gesamtfläche Kallus dorsal}

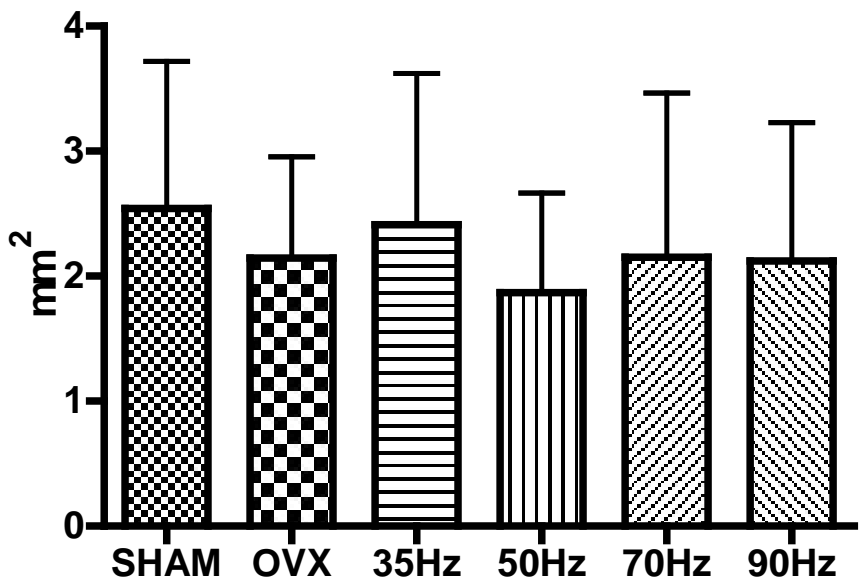

Abbildung 44: Fluoreszenzmikroskopisch ermittelter Messparameter „Gesamtfläche Kallus dorsal“

Dargestellt sind MW \pm SD, signifikante Unterschiede finden sich nicht $(p<0,05$, Tukey-Test

\subsubsection{CG-Fläche Kallus dorsal}

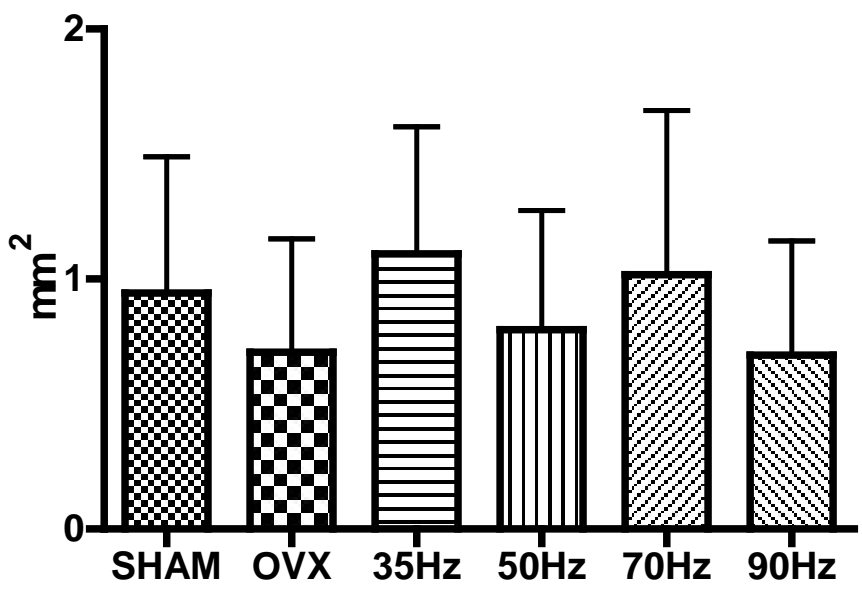

Abbildung 45: Fluoreszenzmikroskopisch ermittelter Messparameter „CG-Fläche Kallus dorsal“

Dargestellt sind MW $\pm S D$, signifikante Unterschiede finden sich nicht $(p<0,05$, Tukey-Test) 


\subsubsection{AK-Fläche Kallus dorsal}

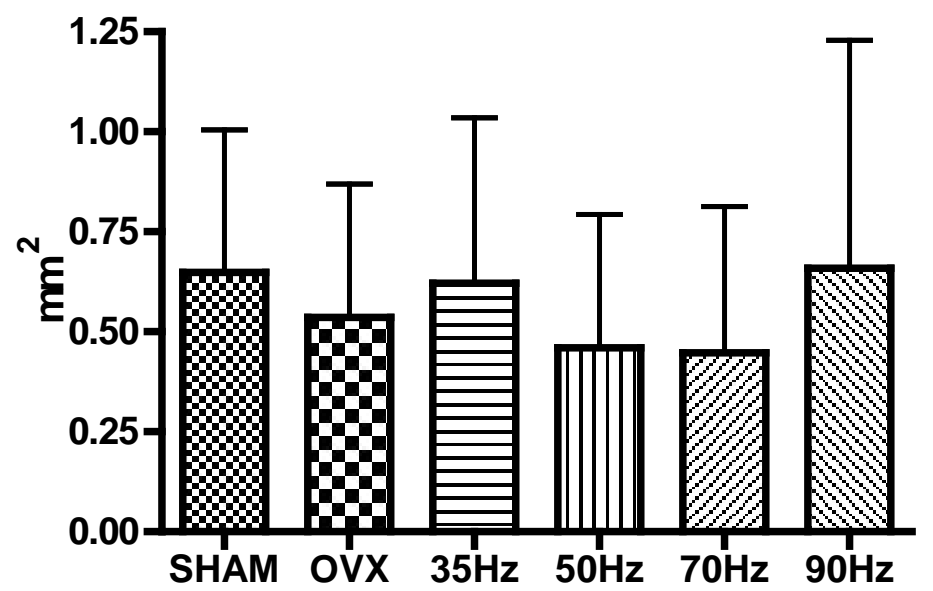

Abbildung 46: Fluoreszenzmikroskopisch ermittelter Messparameter „AK-Fläche Kallus dorsal“"

Dargestellt sind $M W \pm S D$, signifikante Unterschiede finden sich nicht $(p<0,05$, Tukey-Test)

\subsubsection{TC-Fläche Kallus dorsal}

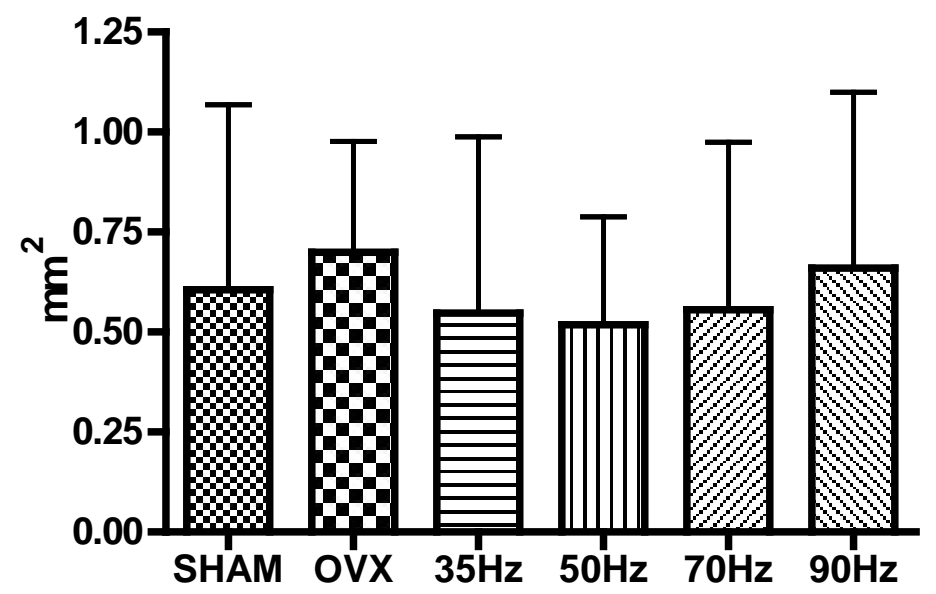

Abbildung 47: Fluoreszenzmikroskopisch ermittelter Messparameter „TC-Fläche Kallus dorsal“"

Dargestellt sind MW $\pm S D$, signifikante Unterschiede finden sich nicht $(p<0,05$, Tukey-Test $)$ 
Bei fluoreszenzmikroskopischer Betrachtung der dorsalen Kallusflächen finden sich zwischen den einzelnen Versuchsgruppen ebenfalls keine signifikanten Unterschiede. Die dorsale Gesamtkallusfläche ist bei der $35 \mathrm{~Hz}-$ Gruppe tendenziell etwas größer als bei den anderen Vibrationsgruppen, auch im die frühe Frakturheilung repräsentierenden CG-Intervall sind die hinteren Kallusflächen bei der $35 \mathrm{~Hz}-\mathrm{Gruppe}$ und bei der $70 \mathrm{~Hz}-$ Gruppe tendenziell größer als bei den anderen Vibrationsgruppen. Bei der $50 \mathrm{~Hz}-G r u p p e$ ist die dorsale Gesamtkallusfläche dagegen tendenziell am kleinsten, auch im frühen CG-Intervall ist die hintere Kallusfläche bei der $50 \mathrm{~Hz}$ Gruppe und der $90 \mathrm{~Hz}-\mathrm{Gruppe}$ tendenziell kleiner als bei den anderen Vibrationsgruppen.

\subsubsection{Kallusfläche endostal}

\subsubsection{Gesamtfläche Kallus endostal}

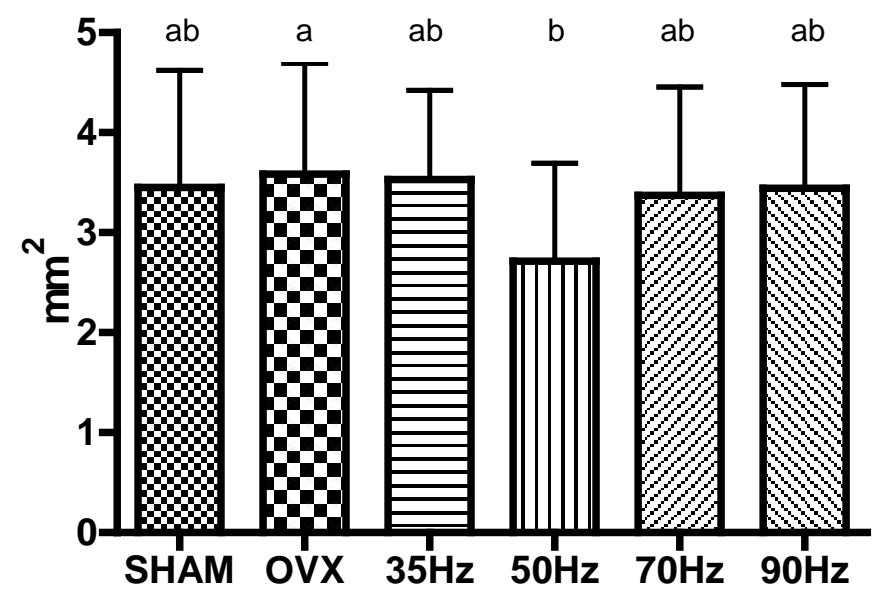

Abbildung 48: Fluoreszenzmikroskopisch ermittelter Messparameter „Gesamtfläche Kallus endostal“"

Dargestellt sind MW \pm SD, MW mit verschiedenen Buchstaben unterscheiden sich signifikant $(p<0,05$, Tukey-Test $)$ 


\subsubsection{CG-Fläche Kallus endostal}

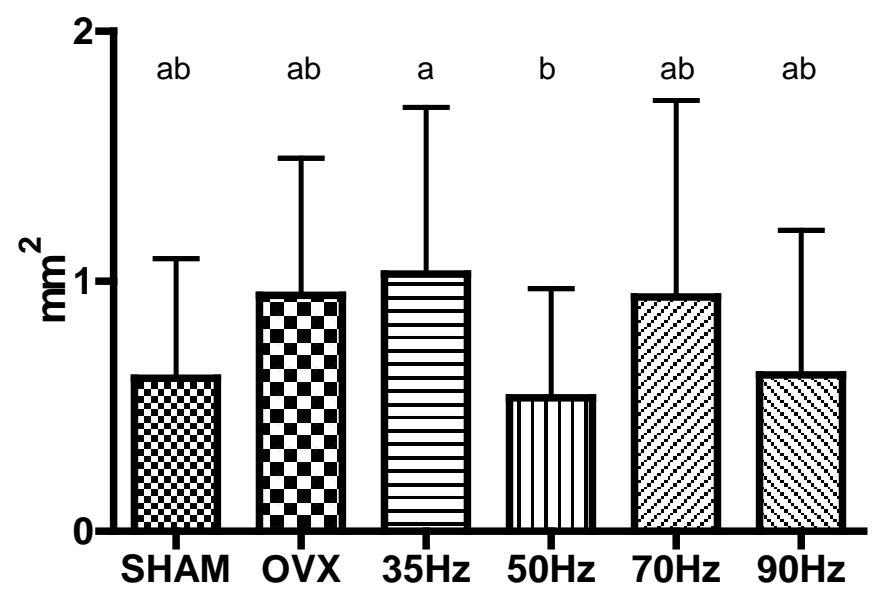

Abbildung 49: Fluoreszenzmikroskopisch ermittelter Messparameter „CG-Fläche Kallus endostal“

Dargestellt sind MW \pm SD, MW mit verschiedenen Buchstaben unterscheiden sich signifikant $(p<0,05$, Tukey-Test)

\subsubsection{AK-Fläche Kallus endostal}

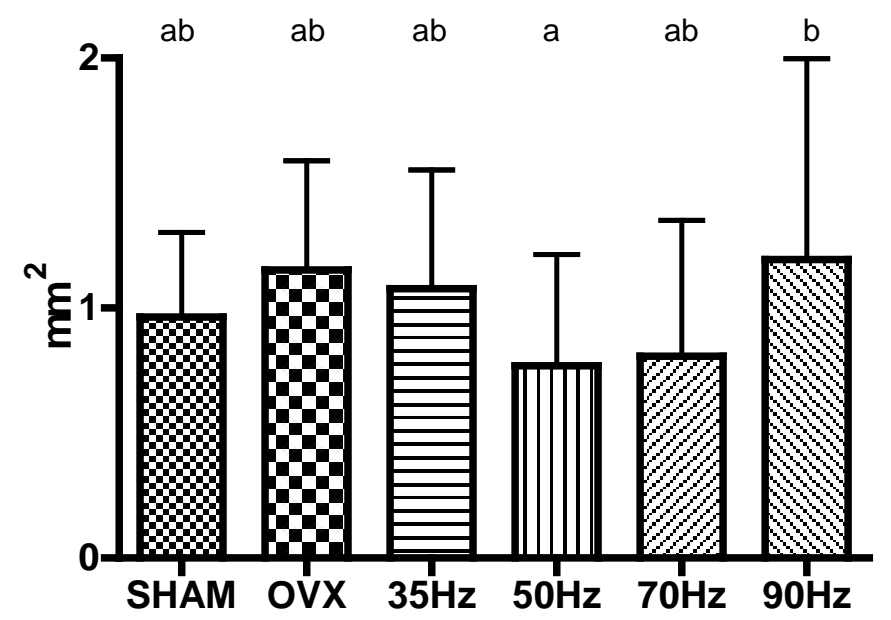

Abbildung 50: Fluoreszenzmikroskopisch ermittelter Messparameter „AK-Fläche Kallus endostal“"

Dargestellt sind MW $\pm S D$, MW mit verschiedenen Buchstaben unterscheiden sich signifikant $(p<0,05$, Tukey-Test $)$ 


\subsubsection{TC-Fläche Kallus endostal}

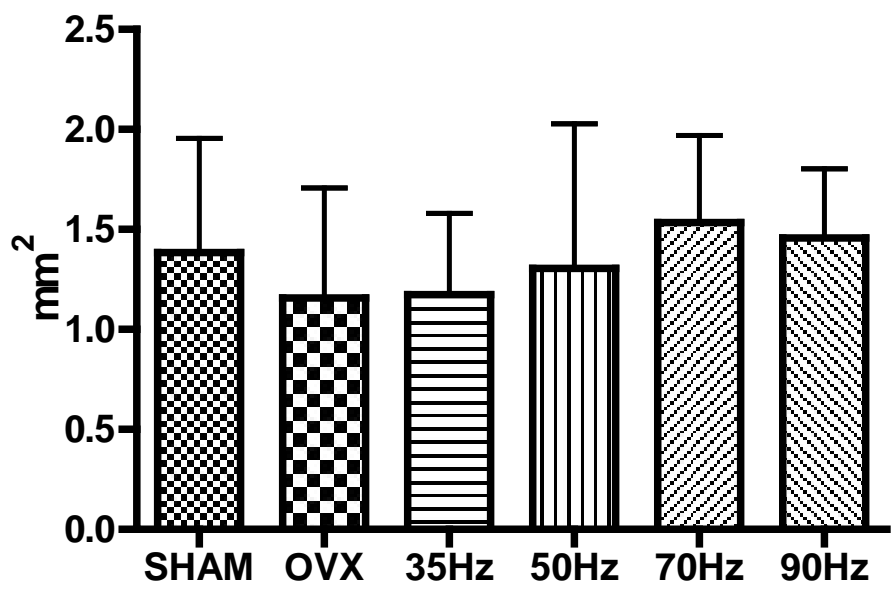

\section{Abbildung 51: Fluoreszenzmikroskopisch ermittelter Messparameter „TC-Fläche Kallus endostal“}

Dargestellt sind MW $\pm S D$, signifikante Unterschiede finden sich nicht $(p<0,05$, Tukey-Test)

Bei fluoreszenzmikroskopischer Betrachtung der endostalen Kallusflächen fällt auf, dass diese im die frühe Frakturheilung repräsentierenden CG-Intervall bei der $35 \mathrm{~Hz}-$ und der $70 \mathrm{~Hz}-$ Gruppe deutlich größer sind als bei der $50 \mathrm{~Hz}-\mathrm{Gruppe}$. Für die $35 \mathrm{~Hz}-$ Gruppe ist diese Differenz auch signifikant.

Die endostale Gesamtkallusfläche der $50 \mathrm{~Hz}-G r u p p e$ ist signifikant kleiner als die der OVX-Gruppe und auch deutlich kleiner als die aller anderen Gruppen, die sich ansonsten nicht wesentlich voneinander unterscheiden. Die endostale CG-Fläche der $50 \mathrm{~Hz}$-Gruppe ist von allen Gruppen ebenfalls am kleinsten, im Vergleich zur $35 \mathrm{~Hz}-$ Gruppe ist die Differenz wie schon erwähnt signifikant. Auch die endostale AK-Fläche der $50 \mathrm{~Hz}-G r u p p e$ ist die kleinste aller Versuchsgruppen mit signifikanter Differenz zur 90Hz-Gruppe. Zwischen den TC-markierten endostalen Kallusflächen sind keine signifikanten Unterschiede erkennbar. 


\subsubsection{Additivkallus}

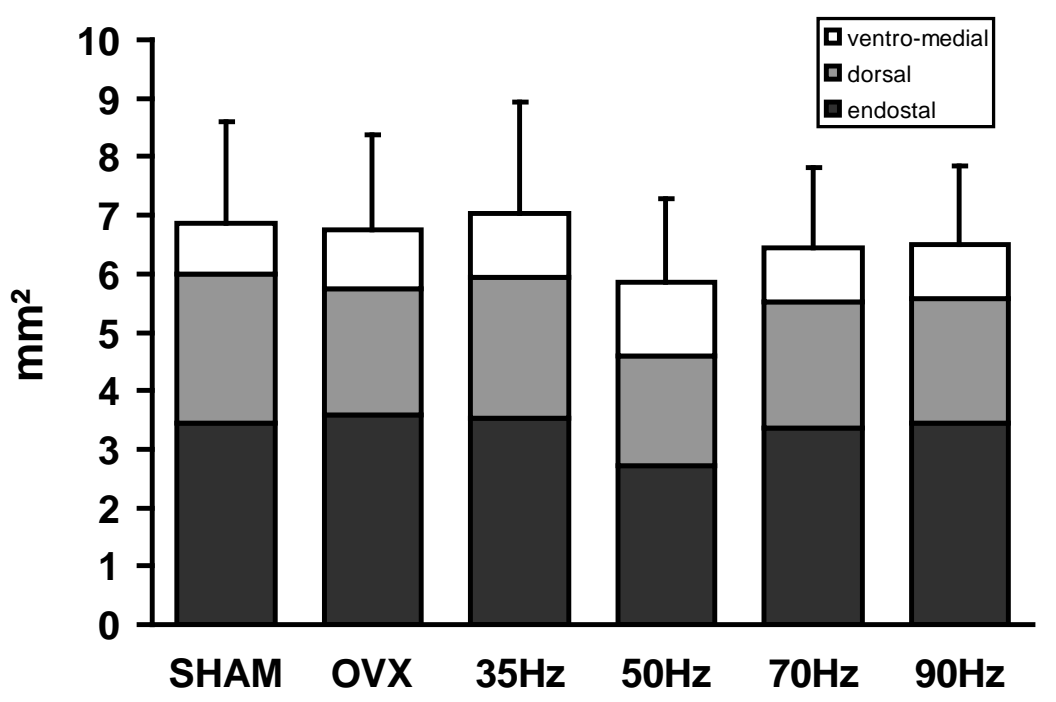

Abbildung 52: Fluoreszenzmikroskopisch ermittelter Messparameter "Additivkallus"

Dargestellt sind $M W \pm S D$, signifikante Unterschiede finden sich nicht $(p<0,05$, Tukey-Test)

Der Additivkallus als Summe der gesamten ventromedialen, dorsalen und endostalen Kallusflächen lässt keine signifikanten Unterschiede zwischen den verschiedenen Versuchsgruppen erkennen. Die Additivkallusfläche der $50 \mathrm{~Hz}-\mathrm{Gruppe}$ ist aber tendenziell etwas kleiner als die der anderen Gruppen.

Bei Vergleich des Gesamtkallus der verschiedenen untersuchten Regionen fällt auf, dass die Kallusfläche aller Versuchsgruppen von ventromedial über dorsal nach endostal deutlich zunimmt. 


\subsection{Tabellarischer Anhang}

Tabelle 7: Tägliche Futteraufnahme [g] der scheinoperierten (SHAM), ovarektomierten (OVX) sowie ovarektomierten und mit verschiedenen Frequenzen vibrierten Tiere im zeitlichen Verlauf als Mittelwerte \pm Standardabweichungen

\begin{tabular}{|c|c|c|c|c|c|c|}
\hline Woche & SHAM & ovx & $\begin{array}{c}\text { OVX } \\
+35 \mathrm{~Hz}\end{array}$ & $\begin{array}{r}\text { OVX } \\
+50 \mathrm{~Hz}\end{array}$ & $\begin{array}{c}\text { ovX } \\
+70 \mathrm{~Hz}\end{array}$ & $\begin{array}{r}\text { OVX } \\
+90 \mathrm{~Hz}\end{array}$ \\
\hline 1 & $\begin{array}{l}18,333 \\
\pm 1,21\end{array}$ & $\begin{array}{l}22,667 \\
\pm 1,37\end{array}$ & $\begin{array}{l}22,581 \\
\pm 0,68\end{array}$ & $\begin{array}{l}23,302 \\
\pm 1,36\end{array}$ & $\begin{array}{l}24,621 \\
\pm 0,78\end{array}$ & $\begin{array}{l}24,343 \\
\pm 0,57\end{array}$ \\
\hline 2 & $\begin{array}{l}18,257 \\
\pm 0,52\end{array}$ & $\begin{array}{l}23,333 \\
\pm 0,92\end{array}$ & $\begin{array}{l}23,571 \\
\pm 0,76\end{array}$ & $\begin{array}{l}24,052 \\
\pm 0,74\end{array}$ & $\begin{array}{l}25,743 \\
\pm 0,73\end{array}$ & $\begin{array}{l}25,810 \\
\pm 0,39\end{array}$ \\
\hline 3 & $\begin{array}{l}18,457 \\
\pm 1,07\end{array}$ & $\begin{array}{l}22,571 \\
\pm 0,37\end{array}$ & $\begin{array}{l}22,543 \\
\pm 1,16\end{array}$ & $\begin{array}{l}23,276 \\
\pm 0,44\end{array}$ & $\begin{array}{c}24,779 \\
\pm 0,74\end{array}$ & $\begin{array}{l}24,114 \\
\pm 0,35\end{array}$ \\
\hline 4 & $\begin{array}{l}17,581 \\
\pm 0,58\end{array}$ & $\begin{array}{l}21,000 \\
\pm 0,83\end{array}$ & $\begin{array}{l}20,590 \\
\pm 1,16\end{array}$ & $\begin{array}{l}21,538 \\
\pm 0,25\end{array}$ & $\begin{array}{l}23,240 \\
\pm 0,58\end{array}$ & $\begin{array}{l}21,981 \\
\pm 0,57\end{array}$ \\
\hline 5 & $\begin{array}{l}17,905 \\
\pm 0,07\end{array}$ & $\begin{array}{l}20,962 \\
\pm 0,56\end{array}$ & $\begin{array}{l}20,552 \\
\pm 0,82\end{array}$ & $\begin{array}{l}20,740 \\
\pm 0,28\end{array}$ & $\begin{array}{l}22,400 \\
\pm 0,79\end{array}$ & $\begin{array}{l}21,200 \\
\pm 0,16\end{array}$ \\
\hline 6 & $\begin{array}{l}17,524 \\
\pm 0,93\end{array}$ & $\begin{array}{l}19,781 \\
\pm 0,77\end{array}$ & $\begin{array}{l}19,514 \\
\pm 0,72\end{array}$ & $\begin{array}{l}19,655 \\
\pm 0,27\end{array}$ & $\begin{array}{l}21,371 \\
\pm 0,89\end{array}$ & $\begin{array}{l}20,248 \\
\pm 0,28\end{array}$ \\
\hline 7 & $\begin{array}{l}17,790 \\
\pm 0,85\end{array}$ & $\begin{array}{l}19,229 \\
\pm 1,27\end{array}$ & $\begin{array}{l}20,119 \\
\pm 2,58\end{array}$ & $\begin{array}{l}18,729 \\
\pm 0,16\end{array}$ & $\begin{array}{c}20,162 \\
\pm 0,76\end{array}$ & $\begin{array}{l}19,724 \\
\pm 0,53\end{array}$ \\
\hline 8 & $\begin{array}{l}14,190 \\
\pm 1,58\end{array}$ & $\begin{array}{l}12,581 \\
\pm 1,41\end{array}$ & $\begin{array}{l}10,386 \\
\pm 4,09\end{array}$ & $\begin{array}{l}12,760 \\
\pm 0,79\end{array}$ & $\begin{array}{l}13,695 \\
\pm 0,74\end{array}$ & $\begin{array}{l}13,400 \\
\pm 1,37\end{array}$ \\
\hline 9 & $\begin{array}{l}17,286 \\
\pm 0,85\end{array}$ & $\begin{array}{l}14,895 \\
\pm 0,31\end{array}$ & $\begin{array}{l}13,536 \\
\pm 0,73\end{array}$ & $\begin{array}{l}14,483 \\
\pm 0,94\end{array}$ & $\begin{array}{l}14,193 \\
\pm 0,28\end{array}$ & $\begin{array}{l}13,467 \\
\pm 0,17\end{array}$ \\
\hline 10 & $\begin{array}{l}17,067 \\
\pm 0,61\end{array}$ & $\begin{array}{l}16,810 \\
\pm 0,18\end{array}$ & $\begin{array}{l}15,852 \\
\pm 0,56\end{array}$ & $\begin{array}{l}16,140 \\
\pm 0,59\end{array}$ & $\begin{array}{l}16,205 \\
\pm 0,55\end{array}$ & $\begin{array}{l}15,505 \\
\pm 0,68\end{array}$ \\
\hline 11 & $\begin{array}{l}17,048 \\
\pm 0,929\end{array}$ & $\begin{array}{l}16,886 \\
\pm 0,35\end{array}$ & $\begin{array}{c}16,238 \\
\pm 0,84\end{array}$ & $\begin{array}{l}16,419 \\
\pm 0,48\end{array}$ & $\begin{array}{l}17,081 \\
\pm 0,84\end{array}$ & $\begin{array}{l}16,724 \\
\pm 0,63\end{array}$ \\
\hline 12 & $\begin{array}{l}21,429 \\
\pm 3,20\end{array}$ & $\begin{array}{l}22,619 \\
\pm 4,32\end{array}$ & $\begin{array}{l}21,990 \\
\pm 3,17\end{array}$ & $\begin{array}{l}21,819 \\
\pm 4,48\end{array}$ & $\begin{array}{c}24,086 \\
\pm 3,44\end{array}$ & $\begin{array}{l}24,124 \\
\pm 3,48\end{array}$ \\
\hline
\end{tabular}


Tabelle 8: Körpergewicht [g] der scheinoperierten (SHAM), ovarektomierten (OVX) sowie ovarektomierten und mit verschiedenen Frequenzen vibrierten Tiere im zeitlichen Verlauf als Mittelwerte \pm Standardabweichungen

\begin{tabular}{|c|c|c|c|c|c|c|}
\hline Woche & SHAM & ovX & $\begin{array}{c}\text { OVX } \\
+35 \mathrm{~Hz}\end{array}$ & $\begin{array}{c}\text { OVX } \\
+50 \mathrm{~Hz}\end{array}$ & $\begin{array}{c}\text { OVX } \\
+70 \mathrm{~Hz}\end{array}$ & $\begin{array}{c}\text { OVX } \\
+90 \mathrm{~Hz}\end{array}$ \\
\hline 1 & $\begin{array}{l}232,667 \\
\pm 17,09\end{array}$ & $\begin{array}{c}239,133 \\
\pm 12,64\end{array}$ & $\begin{array}{c}232,733 \\
\pm 15,83\end{array}$ & $\begin{array}{c}235,500 \\
\pm 7,86\end{array}$ & $\begin{array}{c}252,143 \\
\pm 13,35\end{array}$ & $\begin{array}{c}236,533 \\
\pm 7,78\end{array}$ \\
\hline 2 & $\begin{array}{c}252,333 \\
\pm 16,38\end{array}$ & $\begin{array}{c}256,733 \\
\pm 14,08\end{array}$ & $\begin{array}{c}247,333 \\
\pm 20,51\end{array}$ & $\begin{array}{c}248,643 \\
\pm 10,40\end{array}$ & $\begin{array}{c}265,286 \\
\pm 12,79\end{array}$ & $\begin{array}{c}248,333 \\
\pm 9,04\end{array}$ \\
\hline 3 & $\begin{array}{c}260,466 \\
\pm 16,86\end{array}$ & $\begin{array}{l}289,067 \\
\pm 13,10\end{array}$ & $\begin{array}{l}278,933 \\
\pm 18,23\end{array}$ & $\begin{array}{c}281,786 \\
\pm 12,01\end{array}$ & $\begin{array}{l}296,357 \\
\pm 13,70\end{array}$ & $\begin{array}{l}276,600 \\
\pm 11,26\end{array}$ \\
\hline 4 & $\begin{array}{l}268,400 \\
\pm 15,06\end{array}$ & $\begin{array}{l}310,467 \\
\pm 13,49\end{array}$ & $\begin{array}{l}302,000 \\
\pm 20,37\end{array}$ & $\begin{array}{l}306,143 \\
\pm 11,45\end{array}$ & $\begin{array}{l}324,071 \\
\pm 16,72\end{array}$ & $\begin{array}{c}302,800 \\
\pm 12,69\end{array}$ \\
\hline 5 & $\begin{array}{l}272,266 \\
\pm 16,32\end{array}$ & $\begin{array}{c}319,733 \\
\pm 12,94\end{array}$ & $\begin{array}{l}312,333 \\
\pm 23,26\end{array}$ & $\begin{array}{l}315,714 \\
\pm 11,53\end{array}$ & $\begin{array}{c}334,214 \\
\pm 18,14\end{array}$ & $\begin{array}{l}313,867 \\
\pm 12,02\end{array}$ \\
\hline 6 & $\begin{array}{c}277,466 \\
\pm 13,51\end{array}$ & $\begin{array}{l}322,400 \\
\pm 14,55\end{array}$ & $\begin{array}{l}314,333 \\
\pm 23,32\end{array}$ & $\begin{array}{l}317,429 \\
\pm 11,91\end{array}$ & $\begin{array}{l}340,071 \\
\pm 18,65\end{array}$ & $\begin{array}{l}316,600 \\
\pm 13,13\end{array}$ \\
\hline 7 & $\begin{array}{c}278,333 \\
\pm 14,33\end{array}$ & $\begin{array}{l}328,267 \\
\pm 15,06\end{array}$ & $\begin{array}{l}319,467 \\
\pm 24,06\end{array}$ & $\begin{array}{l}319,929 \\
\pm 11,96\end{array}$ & $\begin{array}{l}344,286 \\
\pm 20,66\end{array}$ & $\begin{array}{c}320,333 \\
\pm 13,99\end{array}$ \\
\hline 8 & $\begin{array}{r}281,067 \\
\pm 13,67\end{array}$ & $\begin{array}{c}330,600 \\
\pm 13,99\end{array}$ & $\begin{array}{l}323,467 \\
\pm 25,16\end{array}$ & $\begin{array}{c}324,571 \\
\pm 12,86\end{array}$ & $\begin{array}{l}349,214 \\
\pm 20,99\end{array}$ & $\begin{array}{l}324,267 \\
\pm 15,12\end{array}$ \\
\hline 9 & $\begin{array}{l}280,400 \\
\pm 17,02\end{array}$ & $\begin{array}{l}325,867 \\
\pm 28,65\end{array}$ & $\begin{array}{c}324,333 \\
\pm 23,38\end{array}$ & $\begin{array}{l}324,929 \\
\pm 11,16\end{array}$ & $\begin{array}{l}349,286 \\
\pm 21,01\end{array}$ & $\begin{array}{c}326,800 \\
\pm 15,19\end{array}$ \\
\hline 10 & $\begin{array}{r}282,733 \\
\pm 17,56 \\
\end{array}$ & $\begin{array}{c}313,533 \\
\pm 16,27\end{array}$ & $\begin{array}{l}305,071 \\
\pm 27,25\end{array}$ & $\begin{array}{c}306,857 \\
\pm 12,22\end{array}$ & $\begin{array}{r}329,357 \\
\pm 19,76\end{array}$ & $\begin{array}{l}309,000 \\
\pm 12,21\end{array}$ \\
\hline 11 & $\begin{array}{l}282,400 \\
\pm 15,99\end{array}$ & $\begin{array}{c}308,733 \\
\pm 14,90\end{array}$ & $\begin{array}{r}294,929 \\
\pm 25,54\end{array}$ & $\begin{array}{c}298,786 \\
\pm 15,70\end{array}$ & $\begin{array}{l}314,571 \\
\pm 18,90\end{array}$ & $\begin{array}{r}296,200 \\
\pm 14,14\end{array}$ \\
\hline 12 & $\begin{array}{c}283,600 \\
\pm 16,78\end{array}$ & $\begin{array}{l}318,600 \\
\pm 17,36\end{array}$ & $\begin{array}{l}301,286 \\
\pm 26,62\end{array}$ & $\begin{array}{l}306,571 \\
\pm 15,57\end{array}$ & $\begin{array}{r}328,857 \\
\pm 22,34\end{array}$ & $\begin{array}{l}300,133 \\
\pm 15,10\end{array}$ \\
\hline 13 & $\begin{array}{c}288,200 \\
\pm 17,43\end{array}$ & $\begin{array}{l}319,467 \\
\pm 15,93\end{array}$ & $\begin{array}{l}307,214 \\
\pm 25,58\end{array}$ & $\begin{array}{l}310,143 \\
\pm 15,90\end{array}$ & $\begin{array}{l}325,500 \\
\pm 21,17\end{array}$ & $\begin{array}{l}304,133 \\
\pm 15,70\end{array}$ \\
\hline 14 & $\begin{array}{l}293,000 \\
\pm 18,13\end{array}$ & $\begin{array}{c}325,533 \\
\pm 18,83\end{array}$ & $\begin{array}{l}312,500 \\
\pm 26,01\end{array}$ & $\begin{array}{c}314,214 \\
\pm 14,78\end{array}$ & $\begin{array}{l}331,071 \\
\pm 20,45\end{array}$ & $\begin{array}{l}309,067 \\
\pm 16,25\end{array}$ \\
\hline
\end{tabular}


Tabelle 9: Ergebnisse der im biomechanischen Biegetest ermittelten qualitativen Parameter Steifheit [N/mm] und maximale Belastbarkeit [N] des Frakturkallus der scheinoperierten (SHAM), ovarektomierten (OVX) sowie ovarektomierten und mit verschiedenen Frequenzen vibrierten Tiere. $\quad M W=$ Mittelwert, SD = Standardabweichung

\begin{tabular}{|c|c|c|c|c|}
\hline \multirow[t]{2}{*}{ Gruppe } & \multicolumn{2}{|c|}{ Steifheit [N/mm] } & \multicolumn{2}{|c|}{ Maximale Belastbarkeit [N] } \\
\hline & MW & SD & MW & SD \\
\hline SHAM & 49,32 & 22,52 & 26,80 & 14,82 \\
\hline ovX & 41,52 & 16,88 & 24,47 & 11,92 \\
\hline OVX+WBV $35 \mathrm{~Hz}$ & 57,40 & 27,55 & 26,83 & 11,35 \\
\hline OVX+WBV $50 \mathrm{~Hz}$ & 58,12 & 24,29 & 24,70 & 11,21 \\
\hline OVX+WBV $70 \mathrm{~Hz}$ & 41,11 & 21,62 & 30,66 & 14,15 \\
\hline OVX+WBV $90 \mathrm{~Hz}$ & 42,45 & 19,16 & 22,69 & 08,46 \\
\hline
\end{tabular}


Tabelle 10: Ergebnisse der quantitativen Analyse der Frakturheilung mittels Micro-CT für scheinoperierte (SHAM), ovarektomierte (OVX) sowie ovarektomierte und mit verschiedenen Frequenzen vibrierte Tiere

Dargestellt sind MW $\pm S D, M W$ mit verschiedenen Buchstaben unterscheiden sich signifikant $(p<0,05$, Tukey-Test)

\begin{tabular}{|c|c|c|c|c|c|c|}
\hline Messparameter & SHAM & ovX & $\begin{array}{r}\text { OVX } \\
+35 \mathrm{~Hz}\end{array}$ & $\begin{array}{r}\text { ovX } \\
+50 \mathrm{~Hz}\end{array}$ & $\begin{array}{r}\text { OVX } \\
+70 \mathrm{~Hz}\end{array}$ & $\begin{array}{r}\text { OVX } \\
+90 \mathrm{~Hz}\end{array}$ \\
\hline $\begin{array}{c}\text { Gesamtknochendichte } \\
{\left[\mathrm{g} / \mathrm{cm}^{3}\right]}\end{array}$ & $\begin{array}{r}1,50 \\
\pm 0,11\end{array}$ & $\begin{array}{r}1,45 \\
\pm 0,17\end{array}$ & $\begin{array}{r}1,37 \\
\pm 0,06\end{array}$ & $\begin{array}{r}1,45 \\
\pm 0,11\end{array}$ & $\begin{array}{c}1,36 \\
\pm 0,04\end{array}$ & $\begin{array}{r}1,38 \\
\pm 0,07\end{array}$ \\
\hline $\begin{array}{c}\text { Kortikalisdichte } \\
{\left[\mathrm{g} / \mathrm{cm}^{3}\right]}\end{array}$ & $\begin{array}{r}1,69 \\
\pm 0,14 \\
\end{array}$ & $\begin{array}{r}1,71 \\
\pm 0,22 \\
\end{array}$ & $\begin{array}{r}1,60 \\
\pm 0,06 \\
\end{array}$ & $\begin{array}{r}1,68 \\
\pm 0,13 \\
\end{array}$ & $\begin{array}{r}1,58 \\
\pm 0,04 \\
\end{array}$ & $\begin{array}{r}1,59 \\
\pm 0,07 \\
\end{array}$ \\
\hline $\begin{array}{c}\text { Kallusdichte } \\
{\left[\mathrm{g} / \mathrm{cm}^{3}\right]}\end{array}$ & $\begin{array}{r}1,36 \\
\pm 0,12 \\
\end{array}$ & $\begin{array}{r}1,35 \\
\pm 0,14 \\
\end{array}$ & $\begin{array}{r}1,27 \\
\pm 0,04 \\
\end{array}$ & $\begin{array}{c}1,34 \\
\pm 0,10 \\
\end{array}$ & $\begin{array}{c}1,27 \\
\pm 0,04\end{array}$ & $\begin{array}{c}1,27 \\
\pm 0,05\end{array}$ \\
\hline $\begin{array}{c}\text { Gesamtknochenvolumen } \\
{\left[\mathrm{mm}^{3}\right]}\end{array}$ & $\begin{array}{l}58,85^{\mathrm{ab}} \\
\pm 10,94\end{array}$ & $\begin{array}{l}55,82^{\mathrm{ab}} \\
\pm 5,36\end{array}$ & $\begin{array}{l}55,90^{\mathrm{ab}} \\
\pm 10,61 \\
\end{array}$ & $\begin{array}{l}53,24^{\mathrm{ab}} \\
\pm 6,51\end{array}$ & $\begin{array}{l}64,47^{\mathrm{a}} \\
\pm 9,09\end{array}$ & $\begin{array}{l}52,39^{\mathrm{b}} \\
\pm 5,84\end{array}$ \\
\hline $\begin{array}{c}\text { Kortikalisvolumen } \\
{\left[\mathrm{mm}^{3}\right]} \\
\end{array}$ & $\begin{array}{l}23,94^{\mathrm{a}} \\
\pm 6,78 \\
\end{array}$ & $\begin{array}{l}16,08^{\mathrm{b}} \\
\pm 2,74 \\
\end{array}$ & $\begin{array}{l}16,85^{\mathrm{b}} \\
\pm 5,78 \\
\end{array}$ & $\begin{array}{l}17,56^{\mathrm{b}} \\
\pm 4,13 \\
\end{array}$ & $\begin{array}{l}19,19^{\mathrm{ab}} \\
\pm 3,38\end{array}$ & $\begin{array}{l}18,10^{\mathrm{ab}} \\
\pm 2,64\end{array}$ \\
\hline $\begin{array}{l}\text { Kallusvolumen } \\
\qquad\left[\mathrm{mm}^{3}\right]\end{array}$ & $\begin{array}{r}34,91 \\
\pm 8,34\end{array}$ & $\begin{array}{r}39,74 \\
\pm 4,91\end{array}$ & $\begin{array}{r}39,05 \\
\pm 11,74\end{array}$ & $\begin{array}{r}35,68 \\
\pm 5,51\end{array}$ & $\begin{array}{r}45,28 \\
\pm 8,64\end{array}$ & $\begin{array}{r}34,29 \\
\pm 6,85\end{array}$ \\
\hline $\begin{array}{c}\text { Gesamtgewebevolumen } \\
{\left[\mathrm{mm}^{3}\right]}\end{array}$ & $\begin{array}{r}103,30 \\
\pm 23,55 \\
\end{array}$ & $\begin{array}{r}120,40 \\
\pm 15,18 \\
\end{array}$ & $\begin{array}{r}108,40 \\
\pm 18,46 \\
\end{array}$ & $\begin{array}{r}98,79 \\
\pm 18,51 \\
\end{array}$ & $\begin{array}{r}128,70 \\
\pm 17,49 \\
\end{array}$ & $\begin{array}{r}104,00 \\
\pm 14,48 \\
\end{array}$ \\
\hline $\begin{array}{l}\text { bone volume fraction } \\
\text { (BV/TV) [\%] }\end{array}$ & $\begin{array}{r}58,44^{\mathrm{a}} \\
\pm 11,81 \\
\end{array}$ & $\begin{array}{l}46,78^{b} \\
\pm 5,12 \\
\end{array}$ & $\begin{array}{l}51,78^{\mathrm{ab}} \\
\pm 6,02 \\
\end{array}$ & $\begin{array}{l}54,78^{\mathrm{ab}} \\
\pm 7,82 \\
\end{array}$ & $\begin{array}{l}50,44^{\mathrm{ab}} \\
\pm 5,88 \\
\end{array}$ & $\begin{array}{r}50,78^{\mathrm{ab}} \\
\pm 5,45 \\
\end{array}$ \\
\hline
\end{tabular}


Tabelle 11: Ergebnisse der quantitativen Analyse der Frakturheilung mittels Mikroradiographie für scheinoperierte (SHAM), ovarektomierte (OVX) sowie ovarektomierte und mit verschiedenen Frequenzen vibrierte Tiere

Dargestellt sind MW $\pm S D, M W$ mit verschiedenen Buchstaben unterscheiden sich signifikant $(p<0,05$, Tukey-Test)

\begin{tabular}{|c|c|c|c|c|c|c|}
\hline Messparameter & SHAM & OVX & $\begin{array}{r}\text { OVX } \\
+35 \mathrm{~Hz}\end{array}$ & $\begin{array}{r}\text { OVX } \\
+50 \mathrm{~Hz}\end{array}$ & $\begin{array}{r}\text { OVX } \\
+70 \mathrm{~Hz}\end{array}$ & $\begin{array}{r}\text { OVX } \\
+90 \mathrm{~Hz}\end{array}$ \\
\hline $\begin{array}{c}\text { Kortikalisdicke } \\
\text { ventromedial }[\mathrm{mm}]\end{array}$ & $\begin{array}{r}0,47 \\
\pm 0,11 \\
\end{array}$ & $\begin{array}{r}0,51 \\
\pm 0,13 \\
\end{array}$ & $\begin{array}{r}0,50 \\
\pm 0,10 \\
\end{array}$ & $\begin{array}{r}0,51 \\
\pm 0,09 \\
\end{array}$ & $\begin{array}{r}0,51 \\
\pm 0,08 \\
\end{array}$ & $\begin{array}{r}0,53 \\
\pm 0,11 \\
\end{array}$ \\
\hline $\begin{array}{l}\text { Kortikalisdicke dorsal } \\
\text { [mm] }\end{array}$ & $\begin{array}{r}0,77 \\
\pm 0,20\end{array}$ & $\begin{array}{r}0,76 \\
\pm 0,20\end{array}$ & $\begin{array}{r}0,68 \\
\pm 0,14\end{array}$ & $\begin{array}{r}0,74 \\
\pm 0,14\end{array}$ & $\begin{array}{r}0,77 \\
\pm 0,16\end{array}$ & $\begin{array}{r}0,80 \\
\pm 0,23\end{array}$ \\
\hline $\begin{array}{c}\text { Kallusdicke } \\
\text { ventromedial [mm] }\end{array}$ & $\begin{array}{l}0,45^{\mathrm{a}} \\
\pm 0,15\end{array}$ & $\begin{array}{l}0,57^{a b} \\
\pm 0,21\end{array}$ & $\begin{array}{l}0,52^{a b} \\
\pm 0,21\end{array}$ & $\begin{array}{l}0,56^{a b} \\
\pm 0,14\end{array}$ & $\begin{array}{r}0,64^{b} \\
\pm 0,20\end{array}$ & $\begin{array}{r}0,62^{b} \\
\pm 0,16\end{array}$ \\
\hline $\begin{array}{l}\text { Kallusdicke } \\
\text { dorsal [mm] }\end{array}$ & $\begin{array}{l}0,71^{\mathrm{a}} \\
\pm 0,21\end{array}$ & $\begin{array}{r}0,74^{\mathrm{a}} \\
\pm 0,16\end{array}$ & $\begin{array}{r}0,76^{\mathrm{a}} \\
\pm 0,26\end{array}$ & $\begin{array}{l}0,89^{a b} \\
\pm 0,24\end{array}$ & $\begin{array}{r}0,94^{\mathrm{b}} \\
\pm 0,26\end{array}$ & $\begin{array}{l}0,79^{\mathrm{ab}} \\
\pm 0,21\end{array}$ \\
\hline $\begin{array}{c}\text { Kortikalisdichte } \\
\text { ventromedial [\%] }\end{array}$ & $\begin{array}{l}99,98^{a} \\
\pm 0,09\end{array}$ & $\begin{array}{l}95,21^{\mathrm{b}} \\
\pm 2,92\end{array}$ & $\begin{array}{l}97,32^{\mathrm{c}} \\
\pm 1,69\end{array}$ & $\begin{array}{l}97,77^{\mathrm{c}} \\
\pm 1,42\end{array}$ & $\begin{array}{l}98,19^{c} \\
\pm 1,23\end{array}$ & $\begin{array}{l}97,29^{c} \\
\pm 1,58\end{array}$ \\
\hline $\begin{array}{c}\text { Kortikalisdichte dorsal } \\
{[\%]}\end{array}$ & $\begin{array}{l}99,99^{\mathrm{a}} \\
\pm 0,02\end{array}$ & $\begin{array}{l}96,55^{\mathrm{b}} \\
\pm 2,04\end{array}$ & $\begin{array}{l}97,98^{\mathrm{cd}} \\
\pm 1,77\end{array}$ & $\begin{array}{l}98,54^{\mathrm{c}} \\
\pm 0,88\end{array}$ & $\begin{array}{l}98,15^{\mathrm{cd}} \\
\pm 1,00\end{array}$ & $\begin{array}{l}97,24^{\mathrm{bd}} \\
\pm 2,01\end{array}$ \\
\hline $\begin{array}{c}\text { Kallusdichte } \\
\text { ventromedial [\%] }\end{array}$ & $\begin{array}{l}85,88^{a} \\
\pm 8,50 \\
\end{array}$ & $\begin{array}{l}71,79^{\mathrm{b}} \\
\pm 9,39 \\
\end{array}$ & $\begin{array}{l}66,36^{\mathrm{b}} \\
\pm 12,18\end{array}$ & $\begin{array}{l}65,15^{b} \\
\pm 15,95\end{array}$ & $\begin{array}{l}71,88^{b} \\
\pm 14,44\end{array}$ & $\begin{array}{l}68,76^{\mathrm{b}} \\
\pm 13,90\end{array}$ \\
\hline $\begin{array}{l}\text { Kallusdichte } \\
\text { dorsal [\%] }\end{array}$ & $\begin{array}{l}91,83^{a} \\
\pm 6,87\end{array}$ & $\begin{array}{l}71,00^{b} \\
\pm 10,83\end{array}$ & $\begin{array}{l}77,40^{\text {bc }} \\
\pm 9,25\end{array}$ & $\begin{array}{l}80,12^{c} \\
\pm 10,14\end{array}$ & $\begin{array}{l}80,57^{\mathrm{c}} \\
\pm 8,19\end{array}$ & $\begin{array}{l}75,92^{\mathrm{bc}} \\
\pm 9,33\end{array}$ \\
\hline $\begin{array}{l}\text { Kallusdichte } \\
\text { endostal [\%] }\end{array}$ & $\begin{array}{l}89,91^{\mathrm{a}} \\
\pm 8,07\end{array}$ & $\begin{array}{l}65,18^{b} \\
\pm 7,94\end{array}$ & $\begin{array}{l}66,78^{\text {bd }} \\
\pm 13,16\end{array}$ & $\begin{array}{l}70,88^{\text {bd }} \\
\pm 12,99\end{array}$ & $\begin{array}{l}81,67^{c} \\
\pm 6,60\end{array}$ & $\begin{array}{l}73,23^{d} \\
\pm 8,15\end{array}$ \\
\hline $\begin{array}{c}\text { Trabekelflächendichte } \\
{[\%]}\end{array}$ & $\begin{array}{l}12,64^{a} \\
\pm 10,64\end{array}$ & $\begin{array}{r}2,00^{\mathrm{b}} \\
\pm 1,88\end{array}$ & $\begin{array}{l}3,06^{b} \\
\pm 3,77\end{array}$ & $\begin{array}{l}3,12^{b} \\
\pm 3,44\end{array}$ & $\begin{array}{l}3,61^{\mathrm{b}} \\
\pm 1,82\end{array}$ & $\begin{array}{r}2,92^{b} \\
\pm 4,86\end{array}$ \\
\hline $\begin{array}{c}\text { Anzahl } \\
\text { Trabekelkreuzungen } \\
\text { absolut }\end{array}$ & $\begin{array}{r}5,19^{\mathrm{a}} \\
\pm 6,29\end{array}$ & $\begin{array}{l}0,33^{b} \\
\pm 0,62\end{array}$ & $\begin{array}{r}0,46^{b} \\
\pm 1,24\end{array}$ & $\begin{array}{r}0,46^{b} \\
\pm 1,02\end{array}$ & $\begin{array}{l}1,19^{b} \\
\pm 1,94\end{array}$ & $\begin{array}{l}0,40^{b} \\
\pm 0,91\end{array}$ \\
\hline $\begin{array}{c}\text { Anzahl } \\
\text { Trabekelkreuzungen } \\
\text { pro } \mathrm{mm}^{2}\left[1 / \mathrm{mm}^{2}\right]\end{array}$ & $\begin{array}{l}1,88^{\mathrm{a}} \\
\pm 2,25\end{array}$ & $\begin{array}{l}0,10^{\mathrm{b}} \\
\pm 1.18\end{array}$ & $\begin{array}{l}0,20^{b} \\
\pm 0,55\end{array}$ & $\begin{array}{r}0,18^{b} \\
\pm 0,40\end{array}$ & $\begin{array}{r}0,30^{b} \\
\pm 0,49\end{array}$ & $\begin{array}{r}0,30^{b} \\
\pm 0,49\end{array}$ \\
\hline $\begin{array}{c}\text { Mittlere Trabekeldicke } \\
\qquad[\mu \mathrm{m}]\end{array}$ & $\begin{array}{l}4,49^{\mathrm{a}} \\
\pm 1,13\end{array}$ & $\begin{array}{r}2,49^{b} \\
\pm 0,95\end{array}$ & $\begin{array}{r}2,46^{b} \\
\pm 1,24\end{array}$ & $\begin{array}{r}2,21^{b} \\
\pm 0,85\end{array}$ & $\begin{array}{r}2,25^{b} \\
\pm 0,51\end{array}$ & $\begin{array}{r}2,47^{\mathrm{b}} \\
\pm 1,01\end{array}$ \\
\hline
\end{tabular}


Tabelle 12: Ergebnisse der chronologischen Analyse der Frakturheilung mittels polychromer Sequenzmarkierung für scheinoperierte (SHAM), ovarektomierte (OVX) sowie ovarektomierte und mit verschiedenen Frequenzen vibrierte Tiere

Dargestellt sind MW \pm SD, MW mit verschiedenen Buchstaben unterscheiden sich signifikant $(p<0,05$, Tukey-Test)

\begin{tabular}{|c|c|c|c|c|c|c|}
\hline Messparameter & SHAM & OVX & $\begin{array}{r}\text { OVX } \\
+35 \mathrm{~Hz}\end{array}$ & $\begin{array}{r}\text { OVX } \\
+50 \mathrm{~Hz}\end{array}$ & $\begin{array}{c}\text { OVX } \\
+70 \mathrm{~Hz}\end{array}$ & $\begin{array}{c}\text { OVX } \\
+90 \mathrm{~Hz}\end{array}$ \\
\hline $\begin{array}{c}\text { Gesamtfläche } \\
\text { Kallus ventromedial } \\
{\left[\mathrm{mm}^{2}\right]}\end{array}$ & $\begin{array}{r}0,88 \\
\pm 0,66\end{array}$ & $\begin{array}{r}1,03 \\
\pm 0,80\end{array}$ & $\begin{array}{r}1,08 \\
\pm 1,03\end{array}$ & $\begin{array}{r}1,26 \\
\pm 0,88\end{array}$ & $\begin{array}{c}0,91 \\
\pm 0,58\end{array}$ & $\begin{array}{r}0,93 \\
\pm 0,49\end{array}$ \\
\hline $\begin{array}{l}\text { CG-Fläche Kallus } \\
\text { ventromedial }\left[\mathrm{mm}^{2}\right]\end{array}$ & $\begin{array}{r}0,26 \\
\pm 0,30 \\
\end{array}$ & $\begin{array}{r}0,29 \\
\pm 0,33 \\
\end{array}$ & $\begin{array}{r}0,20 \\
\pm 0,28\end{array}$ & $\begin{array}{r}0,38 \\
\pm 0,38\end{array}$ & $\begin{array}{r}0,23 \\
\pm 0,22\end{array}$ & $\begin{array}{r}0,31 \\
\pm 0,32\end{array}$ \\
\hline $\begin{array}{l}\text { AK-Fläche Kallus } \\
\text { ventromedial }\left[\mathrm{mm}^{2}\right]\end{array}$ & $\begin{array}{r}0,25 \\
\pm 0,23\end{array}$ & $\begin{array}{r}0,22 \\
\pm 0,21\end{array}$ & $\begin{array}{r}0,26 \\
\pm 0,26\end{array}$ & $\begin{array}{r}0,32 \\
\pm 0,29\end{array}$ & $\begin{array}{r}0,23 \\
\pm 0,23\end{array}$ & $\begin{array}{r}0,27 \\
\pm 0,26\end{array}$ \\
\hline $\begin{array}{l}\text { TC-Fläche Kallus } \\
\text { ventromedial }\left[\mathrm{mm}^{2}\right]\end{array}$ & $\begin{array}{r}0,50 \\
\pm 0,45\end{array}$ & $\begin{array}{r}0,52 \\
\pm 0,34\end{array}$ & $\begin{array}{r}0,35 \\
\pm 0,28\end{array}$ & $\begin{array}{r}0,46 \\
\pm 0,24\end{array}$ & $\begin{array}{r}0,32 \\
\pm 0,27\end{array}$ & $\begin{array}{r}0,30 \\
\pm 0,27\end{array}$ \\
\hline $\begin{array}{c}\text { Gesamtfläche } \\
\text { Kallus dorsal }\left[\mathrm{mm}^{2}\right]\end{array}$ & $\begin{array}{r}2,54 \\
\pm 1,18\end{array}$ & $\begin{array}{r}2,14 \\
\pm 0,81\end{array}$ & $\begin{array}{r}2,41 \\
\pm 1,21\end{array}$ & $\begin{array}{r}1,87 \\
\pm 0,80\end{array}$ & $\begin{array}{r}2,15 \\
\pm 1,31\end{array}$ & $\begin{array}{r}2,12 \\
\pm 1,11\end{array}$ \\
\hline $\begin{array}{c}\text { CG-Fläche Kallus } \\
\text { dorsal }\left[\mathrm{mm}^{2}\right]\end{array}$ & $\begin{array}{r}0,95 \\
\pm 0,55\end{array}$ & $\begin{array}{r}0,71 \\
\pm 0,45\end{array}$ & $\begin{array}{r}1,10 \\
\pm 0,51\end{array}$ & $\begin{array}{r}0,80 \\
\pm 0,48\end{array}$ & $\begin{array}{r}1,02 \\
\pm 0,66\end{array}$ & $\begin{array}{r}0,70 \\
\pm 0,46\end{array}$ \\
\hline $\begin{array}{c}\text { AK-Fläche Kallus } \\
\text { dorsal }\left[\mathrm{mm}^{2}\right]\end{array}$ & $\begin{array}{r}0,65 \\
\pm 0,36\end{array}$ & $\begin{array}{r}0,54 \\
\pm 0,33\end{array}$ & $\begin{array}{r}0,62 \\
\pm 0,41\end{array}$ & $\begin{array}{r}0,46 \\
\pm 0,33\end{array}$ & $\begin{array}{r}0,45 \\
\pm 0,37\end{array}$ & $\begin{array}{r}0,66 \\
\pm 0,57\end{array}$ \\
\hline $\begin{array}{c}\text { TC-Fläche Kallus } \\
\text { dorsal }\left[\mathrm{mm}^{2}\right]\end{array}$ & $\begin{array}{r}0,61 \\
\pm 0,46\end{array}$ & $\begin{array}{r}0,70 \\
\pm 0,28\end{array}$ & $\begin{array}{r}0,55 \\
\pm 0,44\end{array}$ & $\begin{array}{r}0,52 \\
\pm 0,27\end{array}$ & $\begin{array}{r}0,56 \\
\pm 0,42\end{array}$ & $\begin{array}{r}0,66 \\
\pm 0,44\end{array}$ \\
\hline $\begin{array}{c}\text { Gesamtfläche } \\
\text { Kallus endostal } \\
{\left[\mathrm{mm}^{2}\right]}\end{array}$ & $\begin{array}{l}3,45^{\mathrm{ab}} \\
\pm 1,17\end{array}$ & $\begin{array}{r}3,59^{a} \\
\pm 1,11\end{array}$ & $\begin{array}{l}3,53^{a b} \\
\pm 0,89\end{array}$ & $\begin{aligned} & 2,71^{b} \\
\pm & 0,98\end{aligned}$ & $\begin{array}{l}3,37^{\mathrm{ab}} \\
\pm 1,08\end{array}$ & $\begin{array}{l}3,44^{a b} \\
\pm 1,04\end{array}$ \\
\hline $\begin{array}{l}\text { CG-Fläche Kallus } \\
\text { endostal }\left[\mathrm{mm}^{2}\right]\end{array}$ & $\begin{array}{l}0,61^{a b} \\
\pm 0,48 \\
\end{array}$ & $\begin{array}{l}0,94^{a b} \\
\pm 0,55\end{array}$ & $\begin{aligned} & 1,03^{\mathrm{a}} \\
& \pm 0,67\end{aligned}$ & $\begin{array}{r}0,53^{b} \\
\pm 0,44\end{array}$ & $\begin{array}{l}0,94^{a b} \\
\pm 0,79\end{array}$ & $\begin{array}{l}0,63^{\mathrm{ab}} \\
\pm 0,58\end{array}$ \\
\hline $\begin{array}{l}\text { AK-Fläche Kallus } \\
\text { endostal }\left[\mathrm{mm}^{2}\right]\end{array}$ & $\begin{array}{l}0,96^{\mathrm{ab}} \\
\pm 0,34 \\
\end{array}$ & $\begin{array}{l}1,15^{\mathrm{ab}} \\
\pm 0,44\end{array}$ & $\begin{array}{l}1,08^{\mathrm{ab}} \\
\pm 0,48\end{array}$ & $\begin{array}{l}0,77^{a} \\
\pm 0,45\end{array}$ & $\begin{array}{l}0,81^{a b} \\
\pm 0,54\end{array}$ & $\begin{array}{l}1,19^{b} \\
\pm 0,80\end{array}$ \\
\hline $\begin{array}{l}\text { TC-Fläche Kallus } \\
\text { endostal }\left[\mathrm{mm}^{2}\right]\end{array}$ & $\begin{array}{r}1,38 \\
\pm 0,57\end{array}$ & $\begin{array}{r}1,16 \\
\pm 0,55\end{array}$ & $\begin{array}{r}1,17 \\
\pm 0,41\end{array}$ & $\begin{array}{r}1,31 \\
\pm 0,72\end{array}$ & $\begin{array}{r}1,53 \\
\pm 0,44\end{array}$ & $\begin{array}{r}1,46 \\
\pm 0,35\end{array}$ \\
\hline Additivkallus $\left[\mathrm{mm}^{2}\right]$ & $\begin{array}{r}6,88 \\
\pm 1,72\end{array}$ & $\begin{array}{r}6,76 \\
\pm 1,63\end{array}$ & $\begin{array}{r}7,02 \\
\pm 1,92\end{array}$ & $\begin{array}{r}5,85 \\
\pm 1,45\end{array}$ & $\begin{array}{r}6,44 \\
\pm 1,38\end{array}$ & $\begin{array}{r}6,49 \\
\pm 1,36\end{array}$ \\
\hline
\end{tabular}




\section{Diskussion}

\subsection{Diskussion der Methodik}

\subsubsection{Die ovarektomierte Ratte als Osteoporosemodell}

Wie schon unter 1.4.3 dargelegt, gilt die ovarektomierte Ratte als etabliertes Tiermodell für die postmenopausale Osteoporose (Kalu 1991; Wronski und Yen 1991; Frost und Jee 1992) und wird in den Richtlinien der amerikanischen Food and Drug Administration für erste Studien zur Therapie der Osteoporose ausdrücklich empfohlen (Thompson et al. 1995).

Der ovarektomiebedingte Mangel an Sexualhormonen hat in der vorliegenden Untersuchung eine gegenüber der nichtovarektomierten SHAM-Gruppe signifikant vermehrte Futteraufnahme (s. 3.1.1) und eine ebenfalls signifikante Zunahme des Körpergewichts (s. 3.1.2) der Tiere zur Folge, wie sie für ovarektomierte Ratten charakteristisch ist (Komrakova et al. 2009). Auch das im Vergleich zu den gesunden Ratten der SHAM-Gruppe bei allen ovarektomierten Versuchsgruppen um den Faktor 6 verringerte Uterusgewicht (s. 3.1.3) ist ein deutlicher Beleg für den als Folge der Ovarektomie eingetretenen Hormonmangel.

\subsubsection{Das metaphysäre Frakturmodell der ovarektomierten Ratte}

Die meisten Untersuchungen zur Frakturheilung am Osteoporosemodell der Ratte wurden bisher an den Diaphysen des Rattenfemurs (Kubo et al. 1999; Cao et al. 2002; Xu et al. 2003; Shi et al. 2010) bzw. der Rattentibia (Nakajima et al. 2002; Xu et al. 2004; Melhus et al. 2007) durchgeführt, obwohl sich der östrogenmangelinduzierte Knochensubstanzverlust bei der Ratte bevorzugt an der proximalen Tibiametaphyse manifestiert (Wronski et al. 1985) und auch beim Menschen ganz überwiegend die metaphysären Skelettabschnitte vom osteoporotischen Knochenabbau und den in seiner Folge auftretenden Frakturen betroffen sind. 
Die metaphysäre Frakturheilung, die üblicherweise direkt durch endostale Frakturspaltüberbrückung ohne wesentliche periostale Kallusbildung erfolgt, unterscheidet sich grundsätzlich von der diaphysären Knochenheilung, die sich zumeist unter erheblicher periostaler Kallusbildung indirekt vollzieht (Stuermer et al. 2010 b). Dieser grundsätzliche Unterschied im Frakturheilungsmechanismus schien uns ein starkes Argument, unsere Untersuchung an einem metaphysären Frakturmodell durchzuführen und damit an dem Skelettabschnitt, an dem sich osteoporotische Frakturen auch tatsächlich manifestieren. Es war deshalb naheliegend, das von Stuermer und Mitarbeitern in Göttingen entwickelte und etablierte Frakturmodell an der Rattentibiametaphyse (2010 b) einzusetzen. Die vorliegende Untersuchung ist damit unseres Wissens die erste Arbeit, die den Einfluss unterschiedlicher Vibrationsfrequenzen auf die Knochenheilung bei Osteoporose an einem metaphysären Frakturmodell untersucht.

Aus dem für diese Untersuchung gewählten Vorgehen (siehe 2.1.4) resultiert eine stabile Plattenosteosynthese mit einem definierten Osteotomiespalt von $0,5 \mathrm{~mm}$ Breite, der einerseits eine standardisierte Evaluation der endostalen Kallusbildung erlaubt und der andererseits genug Mikrobewegungen zulässt, um auch eine moderate periostale Kallusbildung an der dorsalen Seite zu generieren (Stuermer et al. 2010 b; Daub 2010). Es ist bekannt, dass derart schmale Osteotomiespalten die Frakturheilung tendenziell verbessern, während größere Distanzen die Heilung des Knochens erwartungsgemäß beeinträchtigen (Claes et al. 1997).

Für die Stabilität der Osteosynthese ist die Integrität der Fibula von wesentlicher Bedeutung. Wie Stuermer und Mitarbeiter beobachteten, beeinflusst eine Fibulafraktur durch Induktion einer biomechanischen Instabilität den knöchernen Durchbau der Osteotomie ganz erheblich (2010 b), wodurch der Effekt der Vibrationsbehandlung unzweifelhaft überlagert würde. Wir haben deshalb alle 16 Präparate mit frakturierter Fibula von der Untersuchung ausgeschlossen, unabhängig davon, ob eine Konsolidierung der Osteosynthese erfolgt war oder nicht. 


\subsection{Diskussion der Ergebnisse}

\subsubsection{Qualitative Analyse der Frakturheilung unter vertikaler Ganzkörper- vibration}

Leung und Mitarbeiter hatten hormonell intakte Ratten mit geschlossener Femurfraktur einer $35 \mathrm{~Hz}$-Vibrationsbehandlung unterzogen und dabei eine signifikante Verbesserung der Belastbarkeit des Kallus beobachtet (2009). Tezval und Mitarbeiter konnten bei ovarektomierten Ratten ohne Fraktur die Biegefestigkeit und die Belastbarkeit des proximalen Femurs durch eine $90 \mathrm{~Hz}$-Vibration signifikant verbessern, wobei die entsprechenden Werte der behandelten Ratten das Niveau der hormonell intakten Kontrollgruppe erreichten (2011). Oxlund und Mitarbeiter hatten ovarektomierte Ratten ohne Fraktur mit verschiedenen Frequenzen vibriert und die Tibiadiaphysen dann einem Biegetest unterzogen. Sie konnten dabei die infolge der Ovarektomie verringerte Belastbarkeit der Tibiae durch die Vibrationsfrequenzen $17 \mathrm{~Hz}$ und $45 \mathrm{~Hz}$ signifikant verbessern, nicht aber durch die Frequenz von $30 \mathrm{~Hz}$ (2003).

In der vorliegenden Untersuchung lassen die Streckgrenzen der Kurvenverläufe als Maß für den Beginn der plastischen Verformung des Kallus durch Mikrofrakturen zwischen den einzelnen Versuchsgruppen keine signifikanten Veränderungen erkennen. Die im Vergleich zur SHAM-Gruppe tendenziell erniedrigte Streckgrenze der OVX-Gruppe verdeutlicht aber die infolge der Osteoporose verminderte Belastbarkeit des Kallus. Bei der $70 \mathrm{~Hz}-$ Gruppe erreicht die maximale Belastbarkeit des Kallus deutlich höhere Werte als bei allen anderen Versuchsgruppen einschließlich der hormonell intakten SHAM-Gruppe. Die Vibrationsfrequenz von $70 \mathrm{~Hz}$ ist damit offenbar tendenziell am ehesten geeignet, die Belastbarkeit des Kallus zu verbessern.

Auch die Steigungen der im biomechanischen Biegetest ermittelten Kraft-WegDiagramme als Maß für die Steifheit bzw. Biegefestigkeit des Tibiakallus unterscheiden sich zwischen den einzelnen Versuchsgruppen nicht signifikant. Die 
Ovarektomie hat aber eine tendenzielle Verschlechterung der Biegefestigkeit des Kallus zur Folge, wie der im Vergleich zur SHAM-Gruppe flachere Kurvenverlauf der OVX-Gruppe verdeutlicht. Die $50 \mathrm{~Hz}-$ und die $35 \mathrm{~Hz}-\mathrm{Gruppe}$ zeigen dagegen tendenziell steilere Kurvenverläufe als alle anderen Versuchsgruppen. Die Steifheit bzw. Biegefestigkeit des Kallus verbessert sich also offenbar am ehesten unter einer vertikalen Vibrationsbehandlung mit $50 \mathrm{~Hz}$ bzw. $35 \mathrm{~Hz}$.

Zusammenfassend liefert unser biomechanischer Biegetest deutliche Anhaltspunkte dafür, dass die Kallusbildung bei Osteoporose von der Vibrationsbehandlung in qualitativer Hinsicht profitiert: Die Steifheit bzw. Biegefestigkeit des Kallus wird dabei tendenziell am ehesten durch die Vibrationsfrequenzen von $50 \mathrm{~Hz}$ bzw. $35 \mathrm{~Hz}$ verbessert, die maximale Belastbarkeit des Kallus dagegen durch die Vibrationsfrequenz von $70 \mathrm{~Hz}$.

\subsubsection{Quantitative Analyse der Frakturheilung unter vertikaler Ganzkörper- vibration}

Usui und Mitarbeiter hatten hormonell intakte Kaninchen mit bilateraler Fibulafraktur einer $25 \mathrm{~Hz}$-Vibrationsbehandlung unterzogen und dabei einen gegenüber der nichtvibrierten Kontrollgruppe signifikant verbreiterten Kallus beobachtet (1989). Sehmisch und Mitarbeiter konnten bei ovarektomierten Ratten durch eine $90 \mathrm{~Hz}-$ Vibration alle untersuchten morphologischen Parameter am ersten Lendenwirbelkörper signifikant verbessern (2009). Judex und Mitarbeiter hatten ovarektomierte Ratten ohne Fraktur alternativ mit $45 \mathrm{~Hz}$ und mit $90 \mathrm{~Hz}$ vibriert. Unter der $90 \mathrm{~Hz}-$ Vibration beobachteten sie dabei an der proximalen Tibiametaphyse eine signifikante Steigerung der trabekulären Knochenneubildungsrate sowohl gegenüber der $45 \mathrm{~Hz}-$ Gruppe als auch gegenüber der hormonell intakten Kontrollgruppe (2006).

In der vorliegenden Untersuchung war die quantitative Analyse der Frakturheilung unter vertikaler Ganzkörpervibration sowohl anhand der Mikroradiographie als auch anhand einer Micro-CT-Untersuchung erfolgt. Die mikroradiographische Untersuchung zeigt dabei für die ovarektomierte OVX-Gruppe eine gegenüber der hormonell intakten SHAM-Gruppe signifikante Verringerung der endostalen, dorsalen und ventromedialen Kallusdichte, der dorsalen und ventromedialen Kortikalisdichte 
sowie sämtlicher Trabekelparameter. Die ovarektomiebedingte Osteoporose hat demnach erwartungsgemäß eine signifikante Verschlechterung der Kallusdichte, der Kortikalisdichte und der trabekulären Knochenstruktur zur Folge. Im Unterschied dazu findet sich bei der SHAM-Gruppe ein deutlich dünnerer ventromedialer und dorsaler Kallus als bei allen ovarektomierten Gruppen mit signifikanter Dickedifferenz gegenüber der $70 \mathrm{~Hz}$ - und der $90 \mathrm{~Hz}$-Gruppe ventral sowie gegenüber der $70 \mathrm{~Hz}-$ Gruppe dorsal. Der überraschend dünne, aber besonders dichte Kallus der SHAMGruppe ist offenbar Ausdruck einer direkten, d.h. physiologisch endostalen Frakturspaltüberbrückung ohne wesentliche periostale Kallusbildung bei intakter Ovarienfunktion.

Auch die Micro-CT-Untersuchung zeigt für die OVX-Gruppe eine signifikante Verringerung der Parameter Kortikalisvolumen und „bone-volume-fraction“ und eine tendenzielle Verringerung des Gesamtknochenvolumens im Vergleich zur hormonell intakten SHAM-Gruppe. Es bestätigt sich hier, dass die Ovarektomie durch Induktion einer Osteoporose eine strukturelle Schwächung des Knochens bewirkt. Im Unterschied dazu ist das Kallusvolumen der SHAM-Gruppe etwas kleiner als das der ovarektomierten OVX-Gruppe und auch kleiner als das der meisten Vibrationsgruppen. Das überraschend geringe Kallusvolumen der SHAM-Gruppe kann wiederum als Ausdruck einer direkten Frakturspaltüberbrückung ohne wesentliche periostale Kallusbildung bei intakter Ovarienfunktion gewertet werden. Dass das Kallusvolumen in qualitativer Hinsicht nicht unbedingt ausschlaggebend sein muss, wird auch durch die im Vergleich zur OVX-Gruppe überlegenen biomechanischen Eigenschaften der SHAM-Gruppe in unserem Biegetest verdeutlicht.

Unsere quantitative Analyse bestätigt damit die zu erwartende Verschlechterung der Knochenstruktur infolge der ovarektomiebedingten Osteoporose. Im Folgenden soll nun geprüft werden, ob die osteoporotische Knochenstruktur durch die Vibrationsbehandlung verbessert werden kann. Die untersuchten Vibrationsfrequenzen werden nacheinander einzeln diskutiert. Die Vielzahl der zu prüfenden Parameter erschwert dabei die Übersicht. Der Effekt einer jeden Vibrationsfrequenz wird deshalb zunächst mit der nichtvibrierten OVX-Gruppe verglichen und anschließend mit den anderen untersuchten Frequenzen. Auf diese Weise kann für jede Vibrationsfrequenz 
festgestellt werden, ob sie den osteoporotischen Knochen strukturell verbessern kann und ob sie dabei ggf. effektiver ist als die anderen Frequenzen.

Die $35 \mathrm{~Hz}-$ Vibration verbessert einige morphologische Parameter, wie im Vergleich zur unbehandelten OVX-Gruppe deutlich wird: Im Einzelnen hat sie in der Micro-CT eine Zunahme von Kortikalisvolumen und Gesamtknochenvolumen zur Folge, in der Mikroradiographie eine Erhöhung von Kallusdichte (endostal u. dorsal) und -dicke (dorsal) sowie Kortikalisdichte, Trabekelflächendichte und Trabekelkreuzungsanzahl. Für die dorsale Kallusdicke werden dabei sogar die Werte der hormonell intakten SHAM-Gruppe geringfügig übertroffen.

Während sie im Vergleich zur nichtvibrierten OVX-Gruppe überlegen erscheint, erweist sich die $35 \mathrm{~Hz}$-Vibration im Vergleich mit den anderen Vibrationsfrequenzen als für keinen der in unserer quantitativen Analyse untersuchten Parameter ideal: Endostale Kallusdichte, Kallusdicke und Kortikalisdicke (jeweils ventromedial und dorsal) in der Mikroradiographie sowie Kortikalisvolumen in der Micro-CT erreichen unter dieser Frequenz sogar die schlechtesten Werte aller vibrierten Gruppen. Die Vibrationsfrequenz von $35 \mathrm{~Hz}$, die sich zur Verbesserung der Steifheit des Kallus im biomechanischen Test als verhältnismäßig günstig erwiesen hatte, ist damit für eine Verbesserung der quantitativen Frakturheilungsparameter weniger gut geeignet.

Die 50Hz-Vibration kann den Micro-CT-Parameter Kortikalisvolumen und die Mikroradiographie-Parameter Kallusdichte (endostal u. dorsal) und -dicke (dorsal), Kortikalisdichte (ventromedial u. dorsal), Trabekelflächendichte und Trabekelkreuzungsanzahl gegenüber der nichtvibrierten OVX-Gruppe verbessern; für die dorsale Kortikalisdichte ist diese Verbesserung signifikant. Die entsprechenden Werte der hormonell intakten SHAM-Gruppe werden dabei wieder nur für die dorsale Kallusdicke übertroffen.

Im Vergleich mit den anderen Vibrationsfrequenzen hat die $50 \mathrm{~Hz}-$ Vibration in der Mikroradiographie die stärkste Zunahme der dorsalen Kortikalisdichte zur Folge; die Zunahme ist gegenüber der $90 \mathrm{~Hz}-$ Gruppe signifikant. Auch die Micro-CT-Parameter Kallusdichte, Kortikalisdichte, Gesamtknochendichte und „bone-volume-fraction“ kann die $50 \mathrm{~Hz}$-Vibration geringfügig stärker steigern als die anderen Vibrationsfrequenzen. Nur für die „bone-volume-fraction“ wird dabei allerdings das Niveau der nichtvibrierten OVX-Gruppe übertroffen. Die Vibrationsfrequenz von $50 \mathrm{~Hz}$, die auch 
im biomechanischen Test die Steifheit des Kallus tendenziell am meisten verbessert hatte, steigert damit einzelne morphologische Parameter mehr als die anderen Frequenzen und ist insofern zur quantitativen Stimulation der Frakturheilung zumindest besser geeignet als die Frequenz von $35 \mathrm{~Hz}$.

Die $70 \mathrm{~Hz}-$ Vibration verbessert im Vergleich zur unbehandelten OVX-Gruppe alle Volumenparameter in der Micro-CT und nahezu alle untersuchten MikroradiographieParameter tendenziell (Ausnahmen: Kortikalisdicke ventromedial und mittlere Trabekeldicke). Die strukturelle Verbesserung ist dabei für die Kallusdichte und -dicke (endostal u. dorsal bzw. dorsal) ebenso signifikant wie für die Kortikalisdichte (ventromedial u. dorsal). Durch die $70 \mathrm{~Hz}-$ Vibration werden sogar die Werte der hormonell intakten SHAM-Gruppe für die Kallusdicke (ventromedial u. dorsal) in der Mikroradiographie signifikant übertroffen und für das Kallusvolumen und das Gesamtknochenvolumen in der Micro-CT zumindest tendenziell. Dabei spielt wieder die geringe periostale Kallusbildung bei intakter Ovarienfunktion eine Rolle, auf die oben schon eingegangen wurde.

Im Vergleich mit den anderen Vibrationsfrequenzen bewirkt die $70 \mathrm{~Hz}-$ Vibration die höchste Zunahme aller in der Micro-CT gemessenen Volumina und auch fast aller mikroradiographischen Parameter einschließlich Kallusdichte und -dicke in sämtlichen Lokalisationen (Ausnahmen: Kortikalisdicke ventromedial $u$. dorsal, mittlere Trabekeldicke). Die besonders relevante endostale Kallusdichte übertrifft dabei die Dichtewerte aller anderen Vibrationsgruppen und der nichtvibrierten OVXGruppe signifikant. Die Vibrationsfrequenz von $70 \mathrm{~Hz}$ ist damit zur strukturellen Verbesserung der Frakturheilung des osteoporotischen Knochens am effektivsten. Ihr Effekt auf die qualitativen Frakturheilungsparameter ist allerdings uneinheitlich: Während die maximale Belastbarkeit des Kallus die zumindest tendenziell besten Werte erreicht, ist seine Steifheit bzw. Biegefestigkeit unter dieser Frequenz vergleichsweise niedrig. Eine Erklärung dafür mag die hohe Kortikalisdichte sein, die dem angrenzenden Kallus Elastizität nimmt.

Auch die $90 \mathrm{~Hz}-$ Vibration verbessert im Vergleich zur unbehandelten OVX-Gruppe tendenziell das Kortikalisvolumen und die „bone volume fraction“ in der Micro-CT ebenso wie die meisten mikroradiographischen Parameter (Ausnahmen: Kallusdichte ventromedial, mittlere Trabekeldichte). Für die endostale Kallusdichte ist diese 
strukturelle Verbesserung - wie schon unter der $70 \mathrm{~Hz}-$ Vibration - signifikant. Kallusdicke und Kortikalisdicke (jeweils ventromedial $u$. dorsal) werden dabei durch die $90 \mathrm{~Hz}-$ Vibration sogar über das Niveau der hormonell intakten SHAM-Gruppe hinaus gesteigert, wobei die Zunahme nur für die ventromediale Kallusdicke signifikant ist.

Im Vergleich mit den anderen Vibrationsfrequenzen ist die $90 \mathrm{~Hz}-$ Vibration aber nur für die Kortikalisdicke (ventromedial u. dorsal) und die mittlere Tabekeldicke tendenziell effektiver. Für alle anderen Mikroradiographie-Parameter und für alle Volumenparameter in der Micro-CT ist sie dagegen weniger effektiv als die $70 \mathrm{~Hz}-$ Vibration; für das Gesamtknochenvolumen ist diese Differenz signifikant. Einige Parameter erreichen unter $90 \mathrm{~Hz}$ sogar die schlechtesten Werte aller untersuchten Frequenzen (Kallusvolumen und Gesamtknochenvolumen in der Micro-CT; dorsale Kallusdichte, ventromediale und dorsale Kortikalisdichte, Trabekelflächendichte und absolute Anzahl der Trabekelkreuzungen in der Mikroradiographie). Im biomechanischen Test hatte die $90 \mathrm{~Hz}$-Vibration unter allen Frequenzen die schlechteste Belastbarkeit und die zweitschlechteste Biegefestigkeit des Kallus bewirkt. Der ungünstige Einfluss auf die quantitativen Kallusparameter spiegelt sich damit in der schlechtesten Kallusqualität dieser Vibrationsfrequenz wieder. Offenbar hat die zu intensive biomechanische Stimulation eine Störung der Kalluskalzifikation zur Folge, wie es schon Stuermer und Mitarbeiter beobachtet hatten (2010 a).

Zusammenfassend zeigt auch unsere quantitative Analyse, dass die Frakturheilung bei Osteoporose von der Vibrationsbehandlung in Abhängigkeit von der Frequenz profitiert. Sie liefert deutliche Anhaltspunkte für eine Überlegenheit der Vibrationsfrequenz von $70 \mathrm{~Hz}$, die sich in der unter dieser Frequenz mit Abstand deutlichsten Steigerung von kallösen, kortikalen und auch trabekulären Parametern sowohl in der Micro-CT als auch in der Mikroradiographie manifestiert. Diese strukturellen Verbesserungen spiegeln sich auch in der biomechanischen Qualität wider: Unter allen Frequenzen hatte die $70 \mathrm{~Hz}-$ Vibration die höchste Streckgrenze und damit die höchste Belastbarkeit des Kallus gezeigt, auch wenn dabei das Signifikanzniveau nicht erreicht wurde. 


\subsubsection{Chronologische Analyse der Frakturheilung unter vertikaler Ganzkörpervibration}

Bei Vergleich der im Rahmen der polychromen Sequenzmarkierung ermittelten Gesamtkallusflächen fällt auf, dass die Gesamtkallusfläche von endostal (mit Werten um $3 \mathrm{~mm}^{2}$ ) über dorsal (um $2 \mathrm{~mm}^{2}$ ) nach ventral (um $1 \mathrm{~mm}^{2}$ ) deutlich abnimmt. Dies kann als Indiz für eine physiologisch metaphysäre Frakturheilung gewertet werden, die ja in erster Linie endostal stattfindet.

Die in quantitativer Hinsicht im Vordergrund stehende endostale Kallusfläche ist im die frühe Frakturheilung repräsentierenden CG-Intervall bei der $35 \mathrm{~Hz}$ - und der $70 \mathrm{~Hz}-$ Gruppe deutlich größer als bei der $50 \mathrm{~Hz}$ - und der $90 \mathrm{~Hz}-\mathrm{Gruppe}$; zwischen $35 \mathrm{~Hz}$ - und $50 \mathrm{~Hz}-$ Gruppe ist diese Differenz signifikant. Auch die hintere Kallusfläche, die in quantitativer Hinsicht die zweitgrößte Ausdehnung erreicht, ist im frühen CG-Intervall bei der $35 \mathrm{~Hz}$ - und der $70 \mathrm{~Hz}-$ Gruppe tendenziell größer als bei den anderen Vibrationsgruppen. Vibrationsfrequenzen von $35 \mathrm{~Hz}$ und von $70 \mathrm{~Hz}$ sind somit offenbar am ehesten in der Lage, die frühe Frakturheilung zwischen dem 14. und dem 18. Tag (CG-Intervall) zu stimulieren, zumindest in den Kallusregionen endostal und dorsal, die zusammen rund 5/6 der Gesamtkallusflächen ausmachen (s. Abb. 52 „Additivkallus“).

Im späten TC-Intervall erreicht die quantitativ besonders bedeutende endostale Kallusfläche unter $70 \mathrm{~Hz}$ die tendenziell höchsten Werte. Es wird damit deutlich, dass besonders diese Frequenz die Kallusbildung auch in der späten Phase der Frakturheilung (27. - 35. Tag) noch weiter anregt.

Die endostale Gesamtkallusfläche der $50 \mathrm{~Hz}$-Gruppe ist deutlich kleiner als die der anderen Versuchsgruppen, die sich ansonsten nicht wesentlich voneinander unterscheiden; im Vergleich zur OVX-Gruppe ist die Differenz signifikant. Im CGIntervall zwischen 14. und 18. Tag der Frakturheilung ist die endostale Kallusfläche der $50 \mathrm{~Hz}-$ Gruppe ebenfalls die kleinste aller Gruppen mit signifikanter Differenz zur 35Hz-Gruppe (s.o.). Auch im AK-Intervall zwischen 19. und 26. Tag ist die endostale Kallusfläche der $50 \mathrm{~Hz}-G r u p p e$ von allen Gruppen am kleinsten; im Vergleich zur 
90Hz-Gruppe ist die Differenz signifikant. Die hintere Gesamtkallusfläche ist ebenfalls bei der $50 \mathrm{~Hz}-G r u p p e$ tendenziell am kleinsten, ebenso wie der Additivkallus als Summe der endostalen, der hinteren und der vorderen Gesamtkallusflächen. Im Gegensatz dazu sind vordere Gesamtkallusfläche und vordere Kallusfläche im CGund im AK-Intervall bei der $50 \mathrm{~Hz}-G r u p p e$ tendenziell am größten, was aber weniger ins Gewicht fällt, da die vordere Kallusregion nur 1/6 der Gesamtkallusfläche ausmacht.

Zusammenfassend ergibt die mit Hilfe der polychromen Sequenzmarkierung durchgeführte histomorphometrische Analyse kein einheitliches Bild. Sie lässt aber erkennen, dass Vibrationsfrequenzen von $35 \mathrm{~Hz}$ und $70 \mathrm{~Hz}$ am ehesten geeignet sind, die frühe Frakturheilung zu stimulieren. Durch eine $50 \mathrm{~Hz}$-Vibration scheint dagegen die quantitativ im Vordergrund stehende endostale Kallusbildung eher beeinträchtigt zu werden, während sich unter $70 \mathrm{~Hz}-$ Vibration gerade in der späten Phase der Frakturheilung noch weiter Kallus bildet.

\subsubsection{Vergleichende Analyse der Ergebnisse}

Die im Rahmen dieser Arbeit mit Hilfe des Biegetests durchgeführte qualitative Analyse der Frakturheilung lässt eine Verschlechterung der biomechanischen Eigenschaften des Kallus als Folge der Osteoporose erkennen. Biegefestigkeit und maximale Belastbarkeit des Frakturkallus lassen sich tendenziell durch die Vibrationsbehandlung verbessern, allerdings ergibt sich für die beiden Parameter ein uneinheitliches Bild: Während die Steifheit des Kallus tendenziell von einer Vibration mit $50 \mathrm{~Hz}$ bzw. mit $35 \mathrm{~Hz}$ profitiert, ist die maximale Belastbarkeit des Tibiakallus unter einer Vibrationsbehandlung von $70 \mathrm{~Hz}$ deutlich größer als bei anderen Frequenzen und sogar größer als bei der hormonell intakten SHAM-Gruppe. Da die Belastbarkeit als der klinisch relevante Parameter gilt, sollte aus biomechanischer Sicht sicherlich $70 \mathrm{~Hz}$ präferiert werden.

Auch die chronologische Analyse der Frakturheilung spricht für die $70 \mathrm{~Hz}-$ Vibrationstherapie: Die polychrome Sequenzmarkierung zeigt, dass Vibrationsfrequenzen von $35 \mathrm{~Hz}$ und $70 \mathrm{~Hz}$ die frühe Frakturheilung am besten stimulieren, 
während eine $50 \mathrm{~Hz}$-Vibration gerade die physiologisch endostale Kallusbildung eher verzögert. Die späteren Phasen der Frakturheilung scheinen - nach Erlangen einer Grundstabilität der Kallusformation - eher von höheren Frequenzen, wie $70 \mathrm{~Hz}$ oder $90 \mathrm{~Hz}$, zu profitieren.

Die im Rahmen dieser Untersuchung mittels Mikroradiographie und Micro-CT durchgeführte quantitative Analyse der Frakturheilung lässt eine signifikante Verschlechterung der Kallus- und Kortikalisdichte, des Knochenvolumens und der trabekulären Knochenstruktur infolge der Osteoporose erkennen. Die osteoporosebedingte Schwächung der Knochenstruktur kann durch die Vibrationsbehandlung signifikant verbessert werden. Am effektivsten ist diese strukturelle Verbesserung unter der Vibrationsfrequenz von $70 \mathrm{~Hz}$, die in der Micro-CT unter allen untersuchten Frequenzen die bei weitem positivsten Effekte auf Kallus-, Kortikalis- und Gesamtknochenvolumen hat. Die Mikroradiographie zeigt unter $70 \mathrm{~Hz}$ den mit Abstand dichtesten Kallus und den dichtesten kortikalen und trabekulären Knochen, sie bestätigt ansonsten die Ergebnisse der Micro-CT, wobei das Signifikanzniveau insgesamt häufiger erreicht wird. Für künftige Untersuchungen wird man sich auf eine der beiden morphologischen Methoden beschränken können, da sich die durch sie gewonnenen Erkenntnisse nicht wesentlich unterscheiden. Die Micro-CT bietet den Vorteil eines geringeren zeitlichen und personellen Aufwandes. Die mikroradiographische Untersuchung ist jedoch in der Lage den Knochen feiner zu detektieren, sodass die gemessenen Differenzen häufiger das Signifikanzniveau erreichen.

Als Synthese der mit den verschiedenen Untersuchungsverfahren dieser Arbeit gewonnenen Ergebnisse kann eine überlegene osteoanabole Potenz der Vibrationsfrequenz von $70 \mathrm{~Hz}$ festgestellt werden.

Die Höhe dieser Frequenz erstaunt zunächst insofern, als sie deutlich oberhalb des Frequenzbereichs von $10-50 \mathrm{~Hz}$ liegt, der bei vibrierenden Muskeln natürlicherweise gemessen werden kann (Wakeling und Nigg 2001). Es gibt aber in der Literatur zahlreiche Hinweise auf eine überlegene osteogene Potenz höherer Vibrationsfrequenzen: 
Rubin und Lanyon hatten 1984 (a) eine funktionell isolierte Truthahnulna zunächst mit Drücken stimuliert, die eine Verformung von $2000 \mu$ Strain zur Folge hatten, wie sie bei verschiedenen Spezies unter vermehrter körperlicher Belastung auftritt (Rubin und Lanyon 1984 b). Sie wählten dabei zunächst eine Frequenz von nur 0,5 Hz und konnten so mit einer Behandlungsdauer von täglich 8 Sekunden die Entwicklung einer Inaktivitätsosteoporose verhindern. Nach Erhöhen der Frequenz auf $1 \mathrm{~Hz}$ und der Behandlungsdauer auf 100 s war eine Strainintensität von $1000 \mu$ Strain ausreichend, um einen Knochenabbau zu vermeiden (Rubin und Lanyon 1987). Bei weiterer Steigerung der Frequenz auf $3 \mathrm{~Hz}$ und der Behandlungsdauer auf $600 \mathrm{~s}$ genügten $800 \mu$ Strain, bei 30 Hz über unverändert 600 s waren nur noch $200 \mu$ Strain erforderlich, um einem inaktivitätsbedingten Knochenabbau entgegenzuwirken (Qin et al. 1998). Rubin und Mitarbeiter schlossen aus diesen Beobachtungen, dass die Sensitivität des Knochens gegenüber mechanischer Belastung mit der Frequenz steigt und dass insofern vor allem hochfrequente Vibrationen zur Osteoporoseprophylaxe geeignet sind (2006).

Oxlund und Mitarbeiter vibrierten 2003 ovarektomierte Ratten ohne Fraktur über 90 Tage jeweils 30 Minuten mit Frequenzen von $17 \mathrm{~Hz}, 30 \mathrm{~Hz}$ und $45 \mathrm{~Hz}$. Sie beobachteten dabei die stärkste Knochenneubildung und die höchste Belastbarkeit von Femur und Tibia bei der $45 \mathrm{~Hz}-$ Gruppe als Ausdruck einer deutlichen Überlegenheit der höchsten von ihnen untersuchten Vibrationsfrequenz. Judex und Mitarbeiter behandelten 2007 ovarektomierte Ratten ohne Fraktur 28 Tage lang für jeweils $10 \mathrm{~min}$ mit Frequenzen von $45 \mathrm{~Hz}$ und $90 \mathrm{~Hz}$ und stellten dabei eine Überlegenheit der $90 \mathrm{~Hz}-$ Frequenz fest, unter der eine signifikant höhere trabekuläre und kortikale Knochenneubildung in der proximalen Tibia festzustellen war.

In einer aktuellen Untersuchung setzten Pasqualini und Mitarbeiter (2013) adulte, männliche Ratten ohne Fraktur über 28 Tage jeweils $5 \times 10$ min pro Woche einer Vibration mit Frequenzen von $8 \mathrm{~Hz}, 52 \mathrm{~Hz}$ und $90 \mathrm{~Hz}$ aus. Die 90Hz-Vibration hatte dabei auch bei diesen hormonell intakten Tieren die relativ höchste kortikale und trabekuläre Knochenneubildung an Femur und proximaler Tibia zur Folge und war insofern den anderen Frequenzen klar überlegen, während sich Knochenneubildungsrate und Knochendichte unter der $8 \mathrm{~Hz}$-Vibration sogar verschlechterten. 
Diese an hormonell gesunden, männlichen Ratten durchgeführte Untersuchung erlaubt allerdings nur bedingt Rückschlüsse auf die Frakturheilung bei Osteoporose, zumal zumindest bei weiblichen Tieren erst die Ovarektomie den Knochen für die osteoanabole Wirkung der Vibrationsbehandlung zu sensibilisieren scheint (Rubinacci 2008).

In einer Studie der eigenen Arbeitsgruppe über die metaphysäre Frakturheilung an ovarektomierten Ratten hatten Stuermer und Mitarbeiter 2010 (a) dagegen eher ungünstige Effekte einer $90 \mathrm{~Hz}-$ Vibration festgestellt: Die über 30 Tage jeweils zweimal täglich für 15 Minuten durchgeführte $90 \mathrm{~Hz}-$ Vibration hatte zwar eine Verbesserung der tibialen Kallus- und Kortikalisdichte zur Folge, aber zugleich auch eine deutliche Verschlechterung der biomechanischen Eigenschaften. Die Autoren schlossen daraus auf eine Störung der Kalluskalzifikation durch die zu intensive biomechanische Stimulation.

Eine zusammenfassende Betrachtung der zum Thema vorliegenden Literatur legt den Schluss nahe, dass die zur Stimulation der Knochenneubildung ideale Frequenz im höheren Frequenzbereich bei $90 \mathrm{~Hz}$ oder gering darunter liegt. Die in den vorliegenden Ergebnissen festgestellte Überlegenheit der Vibrationsfrequenz von $70 \mathrm{~Hz}$ lässt sich insofern sehr gut mit den Daten der anderen Autoren in Einklang bringen. Bisher liegen unseres Wissens keine weiteren Untersuchungen über den Effekt einer $70 \mathrm{~Hz}-$ Vibration auf die Frakturheilung vor.

Die im Rahmen der Zielsetzung dieser Arbeit formulierte Frage, ob sich die Frakturheilung bei Osteoporose durch eine Vibrationsbehandlung verbessern lässt, kann abschließend mit ja beantwortet werden. Die Frage, welche Vibrationsfrequenz dazu in besonderer Weise geeignet ist, kann zugunsten der Frequenz von $70 \mathrm{~Hz}$ beantwortet werden. Die Überlegenheit der $70 \mathrm{~Hz}$-Vibration ist allerdings nur in unserer mikroradiographischen Analyse signifikant, während Micro-CT, polychrome Sequenzmarkierung und biomechanische Analyse in unserer Untersuchung zwar stabile, aber lediglich tendenzielle Vorteile der $70 \mathrm{~Hz}-$ Vibration belegen. Die in unserer Arbeit belegte überlegene osteoanabole Potenz der $70 \mathrm{~Hz}-$ Vibration sollte deshalb in Untersuchungen am Großtiermodell verifiziert werden. 


\section{$5 \quad$ Zusammenfassung}

Um experimentell zu klären, ob bestimmte Vibrationsfrequenzen zur osteoanabolen Stimulation des Knochens und speziell zur Verbesserung der Frakturheilung bei Osteoporose geeigneter sind als andere, wurde in dieser Arbeit die Frakturheilung an der proximalen Tibiametaphyse der ovarektomierten Ratte unter einer vertikalen Vibrationsbehandlung mit Frequenzen von 35, 50, 70 und $90 \mathrm{~Hz}$ vergleichend untersucht. Die Tiere wurden dafür nach Tibiaosteotomie und Plattenosteosynthese über 30 Tage für jeweils 15 Minuten der für ihre Gruppe spezifischen Vibrationsfrequenz ausgesetzt. Die Amplitude der Vibration betrug dabei stets $0,5 \mathrm{~mm}$.

Die quantitative Analyse des Frakturkallus mittels Mikroradiographie und Micro-CT ergab deutliche Anhaltspunkte für eine überlegene osteoanabole Potenz der $70 \mathrm{~Hz}$ Vibration in Gestalt einer gegenüber allen anderen Vibrationsgruppen signifikant erhöhten endostalen Kallusdichte sowie einer gegenüber der nichtvibrierten OVXGruppe signifikant erhöhten periostalen Kallusdichte und -dicke. Auch kortikaler und trabekulärer Knochen profitierten von der $70 \mathrm{~Hz}-$ Vibration, was sich in der unter dieser Frequenz - zumindest hinsichtlich der Belastbarkeit - besten Kallusqualität widerspiegelte.

Die $35 \mathrm{~Hz}$-Vibration kann die Kallusbildung ebenfalls anregen und damit auch die biomechanischen Eigenschaften des Kallus (Steifheit) verbessern. Kallus und kortikaler Knochen werden durch $35 \mathrm{~Hz}$ aber schlechter stimuliert als durch alle anderen untersuchten Frequenzen. Die $50 \mathrm{~Hz}$-Vibration verbessert die biomechanischen Eigenschaften in ähnlicher Weise, sie stimuliert Kallus und Kortikalis dabei etwas besser als $35 \mathrm{~Hz}$, aber deutlich schlechter als $70 \mathrm{~Hz}$. Auch die $90 \mathrm{~Hz}-$ Vibration hat einen stimulierenden Effekt auf Kallus, Kortikalis und trabekulären Knochen, insbesondere die Kallusstimulation ist aber wieder deutlich schlechter als unter der Frequenz von $70 \mathrm{~Hz}$. Die $90 \mathrm{~Hz}$-Vibration hat zudem eine übermäßige Reizung des Kallus zur Folge, die sich in den schlechtesten biomechanischen Eigenschaften aller untersuchten Frequenzen manifestiert.

Als Synthese der mit den verschiedenen Untersuchungsverfahren dieser Arbeit gewonnenen Ergebnisse kann eine Überlegenheit der Vibrationsfrequenz von $70 \mathrm{~Hz}$ 
für die Stimulation der Frakturheilung bei Osteoporose festgestellt werden. Diese Ergebnisse am Rattentiermodell sollten - vor Beginn einer klinischen Studie - am Großtiermodell verifiziert werden. 


\section{$6 \quad$ Literaturverzeichnis}

Anderson GL, Limacher M, Assaf AR, Bassford T, Beresford SA, Black H, Bonds D, Brunner R, Brzyski R, Caan B (2004): Effects of conjugated equine estrogen in postmenopausal women with hysterectomy: the Women's Health Initiative randomized controlled trial. JAMA 291(14), 1701-1712

Ann-Lieber M (1987): Bone Histomorphometry: Standardization of nomenclature, symbols, and units. J Bone Miner Res 2 , 595-610

Bartl R, Bartl C (2008): Osteoporose - Prävention, Diagnostik, Therapie. 3. Auflage; Georg Thieme Verlag KG, Stuttgart 2008

Beck BR, Kent K, Holloway L, Marcus R (2006): Novel, high-frequency, low-strain mechanical loading for premenopausal women with low bone mass: early findings. J Bone Miner Metab 24(6), 505-507

Beral V (2003): Breast cancer and hormone-replacement Therapy in the Million Women Study. Lancet 362(9382), 419-427

Beral V, Bull D, Reeves G (2005): Endometrial cancer and hormone-replacement therapy in the Million Women Study. Lancet 365(9470), 1543-1551

Beral V, Bull D, Green J, Reeves G (2007): Ovarian cancer and hormonereplacement therapy in the Million Women Study. Lancet 369(9574), 1703-1710

Black DM, Cummings SR, Karpf DB, Cauley JA, Thompson DE, Nevitt MC, Bauer DC, Genant HK, Haskell WL, Marcus R (1996): Randomised trial of effect of alendronate on risc of fracture in women with existing vertebral fractures. Fracture Intervention Trial Research Group. Lancet 348(9041), 1535-1541

Budsberg SC, Jevens DJ, Brown J, Foutz TL, DeCamp CE, Reece L (1993): Evaluation of limb symmetry indices, using ground reaction forces in healthy dogs. Am J Vet Res 54(10), 1569-1574

Canalis E, Giustina A, Bilezikian JP (2007): Mechanisms of anabolic therapies for osteoporosis. N Engl J Med 357(9), 905-916

Cao Y, Mori S, Mashiba T, Westmore MS, Ma L, Sato M, Akiyama T, Shi L, Komatsubara S, Miyamoto K (2002): Raloxifene, estrogen and alendronate affect the processes of fracture repair differently in ovariectomized rats. J Bone Miner Res $\underline{17}(12), 2237-2246$

Chesnut IC, Skag A, Christiansen C, Recker R, Stakkestad JA, Hoiseth A, Felsenberg D, Huss H, Gilbride J, Schimmer RC (2004): Effects of oral ibandronate administered daily or intermittently on fracture risk in postmenopausal osteoporosis.

J Bone Miner Res 19 
Claes L, Augat P, Suger G, Wilke HJ (1997): Influence of size and stability of the osteotomy gap on the success of fracture healing. J Orthop Res $\underline{15}(4), 577-584$

Cooper C, Atkinson EJ, Jacobsen SJ, O'Fallon WM, Melton LJ $3^{\text {rd }}(1993)$ : Population-based study of survival after osteoporotic fractures. Am J Epidemiol 137(9), 1001-1005

Cummings SR, Black DM, Thompson DE, Applegate WB, Barrett-Connor E, Musliner TA, Palermo L, Prineas R, Rubin SM, Scott JC (1998): Effect of alendronate on risc of fracture in women with low bone density but without vertebral fractures: results from the Fracture Intervention Trial. JAMA 280(24), 2077-2082

Cummings SR, Eckert S, Krueger KA, Grady D, Powles TJ, Cauley JA, Norton L, Nickelsen T, Bjarnason NH, Morrow M (1999): The effect of raloxifen on risc of breast cancer in postmenopausal women: results from the MORE randomized trial. Multiple Outcomes of Raloxifen Evaluation. JAMA 281(23), 2189-2197

Dachverband Osteologie e.V. (2009): DVO-Leitlinie 2009 zur Prophylaxe, Diagnostik und Therapie der Osteoporose bei Erwachsenen. Osteologie $\underline{4}$, 304-328

Daub F: Kurzzeiteffekte von Estradiol, Raloxifen, Phytohormonen und Parathormon auf die metaphysäre Frakturheilung des manifest osteoporotischen Knochens der Ratte. Med. Diss. Göttingen 2010

Dawson-Hughes B, Harris SS, Krall EA, Dallal GE (1997): Effect of calcium and vitamin $D$ supplementation on bone density in men and women 65 years of age or older. N Engl J Med $\underline{337}(10), 670-676$

Delank H-W, Gehlen W: Neurologie. 11. Auflage; Georg Thieme Verlag, Stuttgart 2006

Dutertre M, Smith CL (2000): Molecular mechanism of selective estrogen receptor modulator (SERM) action. J Pharmacol Exp Ther 295(2), 431-437

Ehrlich PJ, Lanyon LE (2002): Mechanical Strain and Bone Cell Function: A Review. Osteoporos Int $\underline{13}, 688-700$

Eisman JA (2001): Good, good, good... good vibrations: the best option for better bones? Lancet $\underline{358}, 1924-1925$

Ettinger B, Black DM, Mitlak BH, Knickerbocker RK, Nickelsen T, Genant HK, Christiansen C, Delmas PD, Zanchetta JR, Stakkestad (1999): Reduction of vertebral fracture risk in postmenopausal women with osteoporosis treated with raloxifen: results from a 3-year randomized clinical trial. Multiple Outcomes of Raloxifen Evaluation (MORE) Investigators. JAMA 282(7), 637-645

Fisk JW, Baigent ML (1975): Clinical and radiological assessment of leg length. N Z Med J $\underline{81}(540), 477-480$ 
Fritton JC, Rubin CT, Qin YX, McLeod KJ (1997): Whole-body vibration in the sceleton: development of a resonance-based testing device. Ann Biomed Eng 25, 831-839

Frost HM (1960): The Utah Paradigm of Skeletal Physiology. International Society of Muskuloskeletal and Neuronal Interactions ISMNI $\underline{1}$

Frost HM (2000): The Utah paradigm of skeletal physiology: an overview of its insights for bone, cartilagine and collagenous tissue organs. $\mathrm{J}$ Bone Miner Metab 18(6), 305-316

Frost HM, Jee WS (1992): On the rat model of human osteopenias and osteoporoses. Bone Miner 18(3), 227-236

Gao Y, Wu X, Terauchi M, Li JY, Grassi F, Galley S, Yang X, Weitzmann MN, Pacifici R (2008): T cells potentiate PTH-induced cortical bone loss through CD40L signaling. Cell Metab $\underline{8}(2), 132-145$

Gennari L, Merlotti D, Valleggi F, Martini G, Nuti R (2007): Selective estrogen receptor modulators for postmenopausal osteoporosis: current state of development. Drugs Aging 24(5), 361-379

Goodship AE, Lawes TJ, Rubin CT (2009): Low-magnitude high-frequency mechanical signals accelerate and augment endochondral bone repair: preliminary evidence of efficacy. J Orthop Res $\underline{27}(7)$, 922-930

Grey A, Reid IR (2006): Differences between the bisphosphonates for the prevention and treatment of osteoporosis. Ther Clin Risk Manag 2(1), 77-86

Häussler B, Gothe H, Gol D, Glaeske D, Pientka L, Felsenberg D (2007): Epidemiology, treatment and costs of osteoporosis in Germany - the Bone EVA Study. Osteoporos Int 18(1), 77-84

Harris ST, Watts NB, Genant HK, McKeever CD, Hangartner T, Keller M, Chesnut $\mathrm{CH} 3^{\text {rd }}$, Brown J, Eriksen EF, Hoseyni MS (1999): Effects of risedronate treatment on vertebral and nonvertebral fractures in women with postmenopausal osteoporosis: a randomized controlled trial. Vertebral Efficacy With Risedronate Therapy (VERT) Study Group. JAMA 282(14), 1344-1352

Hobby B, Lee MA (2013): Managing atrophic nonunion in the geriatric population: incidence, distribution, and causes. Orthop Clin North Am 44(2), 251-256

Johnell O, Kanis JA (2006): An estimate of the worldwide prevalence and disability associated with osteoporotic fractures. Osteoporos Int 17(12), 1726-1733

Johnell O, Kanis JA, Odén A, Sernbo I, Redlund-Johnell I, Petterson C, De Laet C, Jönsson B (2004): Osteoporos Int 15(1), 38-42

Johnson JT, Hansen MS, Wu I, Healy LJ, Johnson CR, Jones GM, Capecchi MR, Keller C (2006): Virtual histology of transgenic mouse embryos for high-throughput phenotyping. PLoS Genet $\underline{2}(4), 471-477$ 
Jones G, Nguyen T, Sambrook PN, Kelly PJ, Gilbert C, Eisman JA (1994): Symtomatic fracture incidence in elderly men and women: the Dubbo Osteoporosis Epidemiology Study (DOES). Osteoporos Int $\underline{4}(5), 277-288$

Judex S, Lei X, Han D, Rubin C (2007): Low-magnitude mechanical signals that stimulate bone formation in the ovariectomized rat are dependent on the applied frequency but not on the strain magnitude. J Biomech $\underline{40}$, 1333-1339

Kalu DN (1991): The ovariectomized rat model of postmenopausal bone loss. Bone Miner 15(3), 175-191

Kanis JA, Johnell $O$ (2005): Requirements for DXA for the management of osteoporosis in Europe. Osteoporos Int 16(3), 229-238

Komrakova M, Werner C, Wicke M, Sehmisch S, Tezval M, Stuermer KM, Stuermer EK (2009): Effects of daidzein, 4-methylbenzylidene camphor or estrogen on gastrocnemius muscle of osteoporotic rats undergoing tibia healing period. $\mathrm{J}$ Endocrinol 201, 253-262

Komrakova M, Stuermer EK, Werner C, Wicke M, Kolios L, Sehmisch S, Tezval M, Daub F, Martens T, Witzenhausen P, Dullin C, Stuermer KM (2010): Effect of human parathyroid hormone hPTH (1-34) applied at different regimes on fracture healing and muscle in ovariectomized and healthy rats. Bone $\underline{47}(3), 480-492$

Kubo T, Shiga T, Hashimoto J, Yoshioka M, Honjo H, Urabe M, Kitajima I, Semba I, Hirasawa $Y$ (1999): Osteoporosis influences the late period of fracture healing in a rat model prepared by ovariectomy and low calcium diet. J Steroid Biochem Mol Biol $\underline{68}(5-6), 197-202$

Lane NE (2006): Epidemiology, etiology, and diagnosis of osteoporosis. Am J Obstet Gynecol 194, 3-11

Leibson CL, Tosteson AN, Gabriel SE, Ransom JE, Melton LJ (2002): Mortality, disability, and nursing home for persons with and without hip fracture: a population-

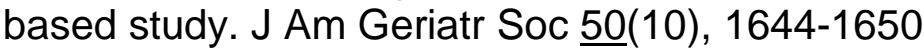

Leung KS, Shi HF, Cheung WH, Qin L, Ng WK, Tam KF, Tang N (2009): Lowmagnitude high-frequency vibration accelerates callus formation, mineralization, and fracture healing in rats. J Orthop Res $\underline{27}(4), 458-465$

Lill CA, Hesseln J, Schlegel U, Eckhardt C, Goldhahn J, Schneider E (2003): Biomechanical evaluation of healing in a non-critical defect in a large animal model of osteoporosis. J Orthop Res 21 (5), 836-842

Mayr H, Ammer K (2007): Ganzkörpervibration GKV) - Methoden und Indikationen. Eine Literaturübersicht. Österr Z Phys Med Rehabil 17(1), 12-22

McClung MR, Geusens P, Miller PD, Zippel H, Bensen WG, Roux C, Adami S, Fogelman I, Diamond T, Eastell R (2001): Effect of risedronate on the risc of hip fracture in eldery women. Hip Intervention Program Study Group. N Engl J Med $\underline{344}$ (5), 333-340 
McNamara LM, Prendergast PJ, Schaffler MB (2005): Bone tissue material properties are altered during osteoporosis. J Musculoskelet Neural Interact $\underline{5}(4), 342-$ 343

Melhus G, Solberg LB, Dimmen S, Madsen JE, Nordsletten L, Reinholt FP (2007): Experimental osteoporosis induced by ovariectomy and vitamin $D$ deficiency does not markedly affect fracture healing in rats. Acta Orthop $\underline{78}(3), 393-403$

Melton LJ $3^{\text {rd }}$, Riggs BL (1985): Risk factors for injury after a fall. Clinics in Geriatric Medicine 1(3), 525-539

Melton LJ $3^{\text {rd }}$, Atkinson EJ, O'Fallon WM, Wahner HW, Riggs BL (1993): Long-term fracture prediction by bone mineral assessed at different skeletal sites. J Bone Miner Res $\underline{8}(10), 1227-1233$

Meunier PJ, Roux C, Seeman E, Ortolani S, Badurski JE, Spector TD, Cannata J, Balogh A, Lemmel EM, Pors-Nielsen S, et al. (2004): The effects of strontium ranelate on the risk of vertebral fracture in women with postmenopausal osteopotosis. N Engl J Med 350(5), 459-468

Nakajima A, Shimoji N, Shimoi K, Shimizu S, Moriya H, Einhorn TA, Yamazaki M (2002): Mechanisms for the enhancement of fracture healing in rats treated with intermittent low-dose human parathyroid hormone (1-34). J Bone Miner Res 17(11), 2038-2047

Namkung-Matthai H, Appleyard R, Jansen J, Hao Lin J, Maastricht S, Swain M, Mason RS, Murrell GAC, Diwan AD, Diamond T (2001): Osteoporosis influences the early period of fracture healing in a rat osteoporotic model. Bone $\underline{28}(1), 80-86$

Nazarov Y, Spivak G (1985): Development of athlete's strength abilities by means of biomechanical stimulation method. Theory and Practice of Physical Culture (Moscow) 12, 445-450

Neer RM, Arnaud CD, Zanchetta JR, Prince R, Gaich GA, Reginster JY, Hodsman $A B$, Eriksen EF, Ish-Shalom S, Genant HK, et al. (2001): Effect of parathyroid hormone (1-34) on fractures and bone mineral density in postmenopausal women with osteoporosis. N Engl J Med 344(19), 1434-1441

NIH Consensus Development Conference (2000): Osteoporosis prevention, diagnosis, and therapy. NIH Consens Statement 17(1), 1-36

Oxlund BS, Ørtoft G, Andreassen TT, Oxlund H (2003): Low-intensity, high-frequency vibration appears to prevent the decrease in strength of the femur and tibia associated with ovariectomy of adult rats. Bone $\underline{32}(1), 69-77$

Particelli F, Mecozzi L, Beraudi A, Montesi M, Baruffaldi F, Viceconti F (2012): A comparison between micro-CT and histology for the evaluation of cortical bone: effect of polymethylmethacrylate embedding on structural parameters. J Microsc $\underline{245}(3), 302-310$ 
Pasqualini M, Lavet C, Elbadaoui M, Vanden Bossche A, Laroche N, Gnyubkin V, Vico L (2013): Skeletal site-specific effects of whole body vibration in mature rats: From deleterious to beneficial frequency-dependent effects. Bone $\underline{55}, 96-77$

Pfeilschifter J: Prophylaxe, Diagnostik und Therapie der Osteoporose bei Frauen ab der Menopause, bei Männern ab dem 60. Lebensjahr. S3-Leitlinie des Dachverbands der Deutschsprachigen Wissenschaftlichen Osteologischen Gesellschaften e.V.. 2. Auflage; Dachverband Osteologie e.V., Essen 2006

Pols HA, Felsenberg D, Hanley DA, Stepan J, Munoz-Torres M, Wilkin TJ, Qin-sheng G, Galich AM, Vandormael K, Yates AJ (1999): Multinational, placebo-controlled, randomized trial of the effects of alendronate on bone density and fracture risk in postmenopausal women with low bone mass: Results of the FOSIT study. Fosamax International Trial Study Group. Osteoporos Int $\underline{9}(5), 461-468$

Qin YX, Rubin CT, McLeod KJ (1989): Nonlinear dependence of loading intensity and cycle number in the maintenance of bone mass and morphology. J Orthop Res $\underline{16}(4), 482-489$

Rahn DA (1976): The fluorochrome sequence labeling of the bone. Nova Acta Leopoldina $\underline{44}$, 249-255

Randell A, Sambrook PN, Nguyen TV, Lapsley H, Jones G, Kelly PJ, Eismann JA 1995): Direct clinical and welfare costs of osteoporotic fractures in elderly men and women. Osteoporos Int $\underline{5}(6), 427-432$

Reginster JY, Sarlet N, Lejeune E, Leonori L (2005): Strontium ranelate: a new treatment for postmenopausal osteoporosis with a dual mode of action. Curr Osteoporos Rep $\underline{3}(1)$, 30-34

Reginster JY, Felsenberg D, Boonen S, Diez Perez A, Rizzoli R, Brandi ML, Spector TD, Brixen K, Goemaere S, Cormier C, et al. (2008): Effects of long-term strontium ranelate treatment on the risk of nonvertebral and vertebral fractures in postmenopausal osteoporosis: Results of a five-year, randomized, placebo-controlled trial. Arthritis Rheum $\underline{58}(6), 1687-1695$

Rossouw JE, Anderson GL, Prentice RL, LaCroix AZ, Kooperberg C, Stefanick ML, Jackson RD, Beresford SA, Howard BV, Johnson KC (2002): Risks and benefits of estrogen plus progestin in healthy postmenopausal women: principal results from the Women`s Health Initiative randomized contolled trial. JAMA 288(3), 321-333

Rubin C, Judex S, Qin YX (2006): Low-level mechanical signals and their potential as a non-pharmacological intervention for osteoporosis. Age Ageing 35 Suppl 2, ii32-ii36

Rubin CT, Lanyon LE (1984 a): Regulation of bone formation by applied dynamic loads. J Bone Joint Surg Am $\underline{66}(3), 397-402$

Rubin CT, Lanyon LE (1984 b): Dynamic strain similarity in vertebrates; an alternative to allometric limb bone scaling. J Theor Biol 107(2), 321-327 
Rubin CT, Lanyon LE (1987): Kappa Delta Award Paper. Osteoregulatory nature of mechanical stimuli: function as a determinant for adaptive remodelling in bone. $\mathrm{J}$ Orthop Res $\underline{5}(2), 300-310$

Rubinacci A, Marenzana M, Cavani F, Colasante F, Villa I, Willnecker J, Moro GL, Spreafico LP, Ferretti M, Guidobono F, et al. (2008): Ovariectomy sensitizes rat

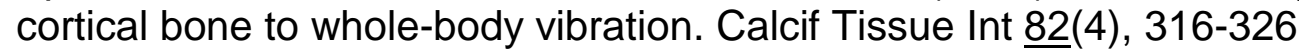

Sehmisch S, Galal R, Kolios L, Tezval M, Dullin C, Zimmer S, Stuermer KM, Stuermer EK (2009): Effects of low-magnitude, high-frequency mechanical stimulation in the rat osteopenia model. Osteoporos Int 20, 1999-2008

Shi HF, Cheung WH, Qin L, Leung AH, Leung KS (2010): Low-magnitude highfrequency vibration treatment augments fracture healing in ovariectomy-induced osteoporotic bone. Bone 46(5), 1299-1305

Smith EL, Gilligan C (1991): Physical activity effects on bone metabolism. Calcif Tissue Int [Suppl] $\underline{49}$, 50-54

Spelsberg TC, Subramaniam M, Riggs BL, Khosla S (1999): The action and interactions of sex steroids and growth factors / cytokines on the skeleton. Mol Endocrinol $\underline{13}(6), 819-828$

Stěpán JJ, Lachman M, Zvěrina J, Pacovský V, Baylink DJ (1989): Castrated men exhibit bone loss: effect of calcitonin treatment on biochemical indices of bone remodeling. J Clin Endocrinol Metab $\underline{69}(3), 523-527$

Stuermer EK, Seidlova-Wuttke D, Sehmisch S, Rack T, Wille J, Frosch KH, Wuttke W, Stuermer KM (2006): Standardized bending and breaking test for the normal and osteoporotic metaphyseal tibias of the rat: effect of estradiol, testosterone, and raloxifene. J Bone Miner Res 21(1), 89-96

Stuermer EK, Komrakova M, Werner C, Wicke M, Kolios L, Sehmisch S, Tezval M, Utesch C, Mangal O, Zimmer S, Dullin C, Stuermer KM (2010 a): Muskoloskeletal response to whole-body vibration during fracture healing in intact and ovariectomized rats. Calcif Tissue Int $\underline{87}, 168-180$

Stuermer EK, Sehmisch S, Rack T, Wenda E, Seidlova-Wuttke D, Tezval M, Wuttke W, Frosch KM, Stuermer KM (2010 b): Estrogen and raloxifene improve metaphyseal fracture healing in the early phase of osteoporosis. A new fracture-healing model at the tibia in rat. Langenbecks Arch Surg 395(2), 163-172

Tezval M, Biblis M, Sehmisch S, Schmelz U, Kolios L, Rack T, Stuermer KM, Stuermer EK (2011): Improvement of femoral bone quality after low-magnitude, highfrequency mechanical stimulation in the ovariectomized rat as an osteopenia model. Calcif Tissue $\underline{88}, 33-40$

Thompson DD, Simmons HA, Pirie CM, Ke HZ (1995): FDA Guidelines and animal models for osteoporosis. Bone 17(4), 125-133 
Usui Y, Zerwekh JE, Vanharanta H, Ashman RB, Mooney V (1989): Different effects of mechanical vibration on bone ingrowth into porous hydroxyapatite and fracture healing in a rabbit model. J Orthop Res $\underline{7}(4), 559-567$

Valencia R, Stuermer EK, Dullin C, Herrmann KP, Kluever I, Zaroban A, Sehmisch S, Funke M, Knollmann F (2006): Erste Erfahrungen mit einem FlächendetektorVolumen-CT (fpVCT) in der experimentellen Osteoporosediagnostik am Kleintiermodell. Radiologe 10, 893-900

Verschueren SM, Roelants M, Delecluse C, Swinnen S, Vanderschueren D, Boonen $S$ (2004): Effect of 6-month whole body vibration training on hip density, muscle strength and postural control in postmenopausal women: a randomized controlled pilot study. J Bone Miner Res $\underline{19}$ (3), 352-359

Wakeling JM, Nigg BM (2001): Soft-tissue vibrations in the quadriceps measured with skin mounted transducers. J Biochem $\underline{34}, 539-543$

Wang JW, Li W, Xu SW, Yang DS, Wang Y, Lin M, Zhao GF (2005): Osteoporosis influences the middle and late periods of fracture healing in a rat osteoporotic model. Chin J Traumatol $\underline{8}(2), 111-116$

WHO (1994): Assessment of fracture risk and its application to screening for postmenopausal osteoporosis. Report of a WHO Study Group. World Health Organ Tech Rep Ser $\underline{843}$, 1-129

Wolff J: Das Gesetz der Transformation der Knochen. Verlag von August Hirschwald, Berlin 1892

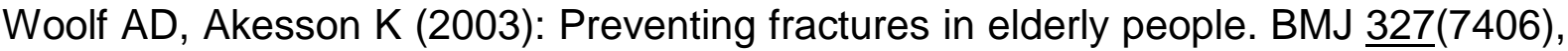
89-95

Wronski TJ, Yen CF (1991): The ovariectomized rat as an animal model for postmenopausal bone loss. Cell Mater $\underline{1}$, 69-74

Wronski TJ, Lowry PL, Walsh CC, Ignaszewski LA (1985): Skeletal alterations in ovariectomized rats. Calcif Tissue Int $\underline{37}(3), 324-328$

Xie L, Jacobson JM, Choi ES, Busa B, Donahue LR, Miller LM, Rubin CT, Judex S (2006): Low-level mechanical vibrations can influence bone resorption and bone formation in the growing skeleton. Bone 39(5), 1059-1066

Xie L, Rubin C, Judex S (2008): Enhancement of the adolescent murine musculoskeletal system using low-level mechanical vibrations. J Appl Physiol 104(4), 1056-1062

Xu SW, Yu R, Zhao GF, Wang JW (2003): Early period of fracture healing in ovariectomized rats. Chin J Traumatol $\underline{6}(3), 160-166$

Xu SW, Wang JW, Li W, Wang Y, Zhao GF (2004): Osteoporosis impairs fracture healing of tibia in a rat osteoporotic model. Zhonghua Yi Xue Za Zhi 84(14), 12051209 


\section{$7 \quad$ Tabellenverzeichnis}

Tabelle 1: Gruppenzuordnung der Tiere.... 15

Tabelle 2: Messparameter der Micro-CT-Untersuchung

Tabelle 3: Mikroradiographisch ermittelte Messparameter und ihre Berechnung.....

Tabelle 4: Dosierung, Applikationszeitpunkt, markierte Zeitspanne und Farbe der einzelnen Fluorochrome

Tabelle 5: Messparameter der polychromen Sequenzmarkierung

Tabelle 6: Uterusgewicht der scheinoperierten, ovarektomierten sowie ovarektomierten und mit verschiedenen Frequenzen vibrierten Tiere am Tag der Obduktion

Tabelle 7: Tägliche Futteraufnahme der scheinoperierten, ovarektomierten sowie ovarektomierten und mit verschiedenen Frequenzen vibrierten Tiere im zeitlichen Verlauf.

Tabelle 8: Körpergewicht der scheinoperierten, ovarektomierten sowie ovarektomierten und mit verschiedenen Frequenzen vibrierten Tiere im zeitlichen Verlauf.

Tabelle 9: Ergebnisse der im biomechanischen Biegetest ermittelten qualitativen Parameter Steifheit und maximale Belastbarkeit des Frakturkallus der scheinoperierten, ovarektomierten sowie ovarektomierten und mit verschiedenen Frequenzen vibrierten Tiere

Tabelle 10: Ergebnisse der quantitativen Analyse der Frakturheilung mittels Micro-CT für scheinoperierte, ovarektomierte sowie ovarektomierte und mit verschiedenen Frequenzen vibrierte Tiere....

Tabelle 11: Ergebnisse der quantitativen Analyse der Frakturheilung mittels Mikroradiographie für scheinoperierte, ovarektomierte sowie ovarektomierte und mit verschiedenen Frequenten vibrierte Tiere

Tabelle 12: Ergebnisse der chronologischen Analyse der Frakturheilung mittels polychromer Sequenzmarkierung für scheinoperierte, ovarektomierte sowie ovarektomierte und mit verschiedenen Frequenzen vibrierte Tiere. .90 


\section{Abbildungsverzeichnis}

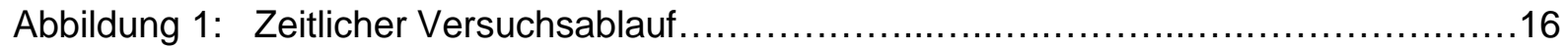

Abbildung 2: Osteotomierte Rattentibia im Röntgenbild ap und seitlich mit

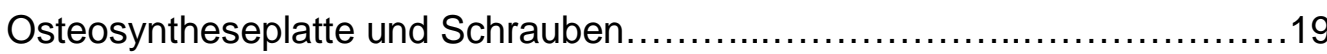

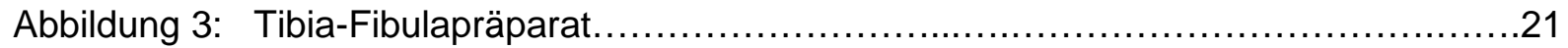

Abbildung 4: Röntgenbild einer Rattentibia ap und seitlich..................................

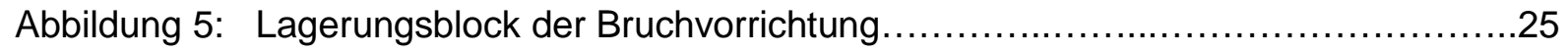

Abbildung 6: Versuchsaufbau des Biegetests mit Bruchvorrichtung und Tibia.................26

Abbildung 7: Geöffneter Probenhalter mit Tibiae und Dichtephantom.......................28

Abbildung 8: Rattentibia mit Messrahmen im 3DOsteoAnalyze-Programm...................30

Abbildung 9a: Viergipfliges Histogramm mit den Grauwertbereichen von Luft,

Weichteilgewebe, nichtkortikalem Knochen und kortikalem Knochen..........31

Abbildung 9b: Dreigipfliges Histogramm mit Grauwertbereichen von Weichteilgewebe, nichtkortikalem und kortikalem Knochen....................................31

Abbildung 10: Darstellung des nichtkortikalen Knochens im Histogramm und im OsteoAnalyze-Programm.

Abbildung 11: Digitalisierte Mikroradiographie mit Kennzeichnung der räumlichen Ausrichtung.....

Abbildung 12: Graudetektion vor und nach manueller Korrektur..........................38

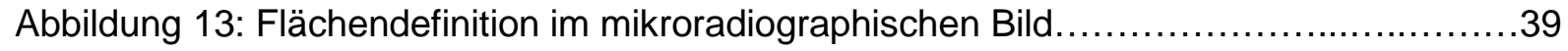

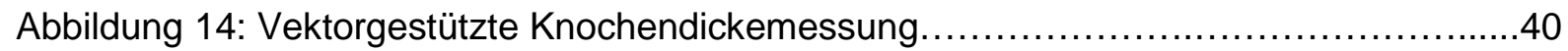

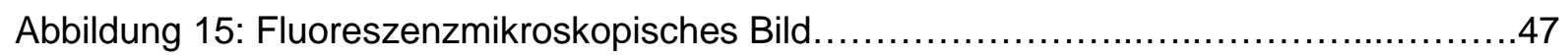

Abbildung 16: Durchschnittliche tägliche Futteraufnahme der Tiere im Verlauf...............50

Abbildung 17: Durchschnittliches Körpergewicht der Tiere im Verlauf......................51

Abbildung 18: Steifheit des Tibiakallus repräsentiert durch die Steigung des

Kurvenverlaufs im biomechanischen Biegetest.

Abbildung 19: Maximale Belastbarkeit des Tibiakallus repräsentiert durch die

im Kurvenverlauf des biomechanischen Biegetests ermittelte

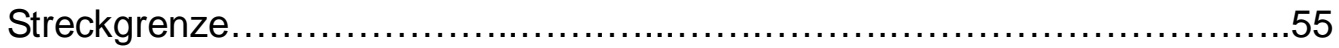

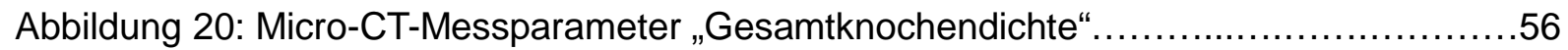

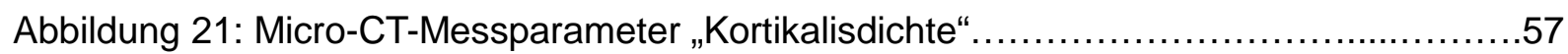




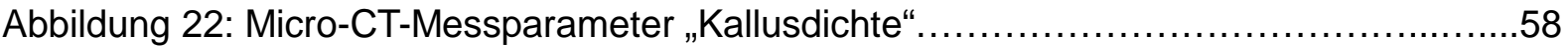

Abbildung 23: Micro-CT-Messparameter „Gesamtknochenvolumen“ $\ldots \ldots \ldots \ldots \ldots \ldots \ldots \ldots \ldots \ldots$

Abbildung 24: Micro-CT-Messparameter „Kortikalisvolumen“...............................60

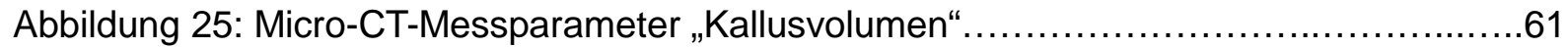

Abbildung 26: Micro-CT-Messparameter „bone volume fraction“ $\ldots \ldots \ldots \ldots \ldots \ldots \ldots \ldots \ldots \ldots \ldots 2$

Abbildung 27: Mikroradiographisch ermittelter Messparameter „Kortikalisdicke

ventromedial“".....

Abbildung 28: Mikroradiographisch ermittelter Messparameter „Kortikalisdicke dorsal“

Abbildung 29: Mikroradiographisch ermittelter Messparameter „Kallusdicke

ventromedial“ .65

Abbildung 30: Mikroradiographisch ermittelter Messparameter „Kallusdicke dorsal“.... 66

Abbildung 31: Mikroradiographisch ermittelter Messparameter „Kortikalisdichte

ventromedial“

Abbildung 32: Mikroradiographisch ermittelter Messparameter „Kortikalisdichte dorsal“. .68

Abbildung 33: Mikroradiographisch ermittelter Messparameter „Kallusdichte ventromedial“ 69

Abbildung 34: Mikroradiographisch ermittelter Messparameter „Kallusdichte dorsal“.... .70

Abbildung 35: Mikroradiographisch ermittelter Messparameter „Kallusdichte endostal“......71

Abbildung 36: Mikroradiographisch ermittelter Messparameter „Trabekelflächendichte“.....72

Abbildung 37: Mikroradiographisch ermittelter Messparameter „Anzahl

Trabekelkreuzungen absolut"

Abbildung 38: Mikroradiographisch ermittelter Messparameter „Anzahl

Trabekelkreuzungen pro $\mathrm{mm}^{2 ،}$ .74

Abbildung 39: Mikroradiographisch ermittelter Messparameter „Mittlere Trabekeldicke“......75

Abbildung 40: Fluoreszenzmikroskopisch ermittelter Messparameter „Gesamtfläche Kallus ventromedial“.

Abbildung 41: Fluoreszenzmikroskopisch ermittelter Messparameter „CG-Fläche

Kallus ventromedial“

Abbildung 42: Fluoreszenzmikroskopisch ermittelter Messparameter „AK-Fläche

Kallus ventromedial“

Abbildung 43: Fluoreszenzmikroskopisch ermittelter Messparameter „TC-Fläche Kallus ventromedial“ 
Abbildung 44: Fluoreszenzmikroskopisch ermittelter Messparameter „Gesamtfläche Kallus dorsal“ .79

Abbildung 45: Fluoreszenzmikroskopisch ermittelter Messparameter „CG-Fläche Kallus dorsal“ .79

Abbildung 46: Fluoreszenzmikroskopisch ermittelter Messparameter „AK-Fläche Kallus dorsal“

Abbildung 47: Fluoreszenzmikroskopisch ermittelter Messparameter „TC-Fläche Kallus dorsal“ .80

Abbildung 48: Fluoreszenzmikroskopisch ermittelter Messparameter „Gesamtfläche Kallus endostal"

Abbildung 49: Fluoreszenzmikroskopisch ermittelter Messparameter „CG-Fläche Kallus endostal“....... 82

Abbildung 50: Fluoreszenzmikroskopisch ermittelter Messparameter „AK-Fläche Kallus endostal“.....

Abbildung 51: Fluoreszenzmikroskopisch ermittelter Messparameter „TC-Fläche Kallus endostal" .83

Abbildung 52: Fluoreszenzmikroskopisch ermittelter Messparameter „Additivkallus“... .84 


\section{Danksagung}

Frau Prof. Dr. med. Ewa Stürmer hat mir das interessante Thema dieser Untersuchung überlassen und meine Arbeit daran durch ihre konstruktive Kritik und ihre schnellen Korrekturen ganz maßgeblich vorangetrieben. Für ihre engagierte Betreuung bedanke ich mich ganz herzlich.

Auch Frau Dr. rer. nat. Marina Komrakova möchte ich danken, die immer für mich ansprechbar war, mir viele wertvolle Anregungen gegeben und mich insbesondere bei der Auswertung der Ergebnisse unterstützt hat.

Mein Dank gilt außerdem Herrn Christian Dullin, der mich in die Micro-CT und speziell in das von inm entwickelte 3DOsteoAnalyze-Programm eingearbeitet hat.

Dankbar bin ich des Weiteren dem kürzlich verstorbenen Herrn Dr. med. Thomas Rack für die Programmierung der Software zur Auswertung der Mikroradiographien.

Nicht zuletzt danke ich den Mitarbeiterinnen und Mitarbeitern des Labors, Annette Witt, Ramona Castro-Machguth und Fritz Kauer, die mit ihrer Hilfsbereitschaft und freundlichen Unterstützung einen Großteil zur Fertigstellung dieser Arbeit beigetragen haben. 